NBER WORKING PAPER SERIES

THE ECONOMIC CASE FOR GLOBAL VACCINATIONS: AN EPIDEMIOLOGICAL MODEL WITH INTERNATIONAL PRODUCTION NETWORKS

\author{
Cem Çakmaklı \\ Selva Demiralp \\ Șebnem Kalemli-Özcan \\ Sevcan Yeşiltaş \\ Muhammed A. Yildırım \\ Working Paper 28395 \\ http://www.nber.org/papers/w28395
}

\author{
NATIONAL BUREAU OF ECONOMIC RESEARCH \\ 1050 Massachusetts Avenue \\ Cambridge, MA 02138 \\ January 2021
}

We are privileged to have received late Emmanuel Farhi's feedback on earlier drafts. We thank David Baqaee, Nitya Pandalai-Nayar and Alvaro Silva for their insightful comments. We thank Yasin Şimşek for excellent research assistance and Ayşe Nur Demiralp for the quote in the preamble. We thank seminar participants at NBER, IMF, ECB, CEPR, EIB, Central Bank of Chile, OECD, the World Bank and the ERF for their comments. We acknowledge support from ICC Research Foundation. The views expressed herein are those of the authors and do not necessarily reflect the views of the National Bureau of Economic Research.

NBER working papers are circulated for discussion and comment purposes. They have not been peerreviewed or been subject to the review by the NBER Board of Directors that accompanies official NBER publications.

(C) 2021 by Cem Çakmakl1, Selva Demiralp, Șebnem Kalemli-Özcan, Sevcan Yeşiltaş, Muhammed A. Yıldırım. All rights reserved. Short sections of text, not to exceed two paragraphs, may be quoted without explicit permission provided that full credit, including $\odot$ notice, is given to the source. 
The Economic Case for Global Vaccinations: An Epidemiological Model with International Production Networks

Cem Çakmaklı, Selva Demiralp, Șebnem Kalemli-Özcan, Sevcan Yeşiltaş, and Muhammed A. Yildirım

NBER Working Paper No. 28395

January 2021

JEL No. F0,F15,F16,F2

\author{
$\underline{\text { ABSTRACT }}$ \\ We develop a multi-sector-country model with input-output linkages to study the effects of \\ sectoral demand and supply shocks within the global trade and production network. Using the \\ model, we quantify output losses of advanced economies (AEs) stemming from lack of \\ vaccinations in the emerging markets and developing economies (EMDEs) during Covid-19. The \\ sectoral shocks for 65 countries and 35 sectors are based on changes in sectoral consumption \\ demand and labor supply as a function of infections. Endogenous lockdowns triggered by lack of \\ vaccinations in EMDEs hurt AEs via a shortage of intermediate inputs, higher import prices, and \\ weak demand for their exports. We provide upper and lower bound estimates for negative output \\ effects of global supply chain disruptions, depending on the degree of complementarity across \\ factors of production. Vaccinating EMDEs is a high return investment for AEs to smooth out the \\ economic impact of the pandemic in their home countries. \\ Cem Çakmaklı \\ Koç University \\ College of Administrative \\ Sciences and Economics \\ Rumelifener Yolu \\ Sariyer, Istanbul \\ Turkey \\ ccakmakli@ku.edu.tr \\ Selva Demiralp \\ Koç University \\ Rumelifeneri Yolu, Sariyer \\ Istanbul 34450 \\ Turkey \\ sdemiralp@ku.edu.tr \\ Șebnem Kalemli-Özcan, \\ Department of Economics \\ University of Maryland \\ Tydings Hall 4118D \\ College Park, MD 20742-7211 \\ and CEPR \\ and also NBER \\ kalemli@econ.umd.edu \\ Sevcan Yeşiltaş \\ Koç University \\ Department of Economics and Finance \\ Rumelifeneri Yolu 34450 \\ Sariyer, Istanbul \\ Turkey \\ syesiltas@ku.edu.tr \\ Muhammed A. Yildırım \\ Koc University Rumelifeneri Yolu \\ Sariyer Istanbul 34450 \\ TURKEY \\ yildirim@gmail.com
}




\section{No Man is an Island}

"No man is an island entire of itself; every man is a piece of the continent, a part of the main; if a clod be washed away by the sea, Europe is the less, as well as if a promontory were, as well as any manner of thy friends or of thine own were; any man's death diminishes me, because I am involved in mankind. And therefore never send to know for whom the bell tolls; it tolls for thee."

- John Donne

\section{Introduction}

On January 28, 2021, Taiwan sought Germany's help in securing Covid-19 vaccines, after Berlin asked for the island's assistance in easing a shortage of automobile semiconductor chips. ${ }^{1}$ Taiwan's move was a strategic attempt to swap two vital shortages at the time: The shortage of vaccines and the shortage of chips. If Germany wanted chips for its automobile industry, she needed to deliver enough vaccines to limit the spread of the pandemic in Taiwan, so that the chips could be produced and delivered to Germany.

In a year, these shortages spread to other sectors, encompassing all factors of production leading to the highest levels of global inflation in the last four decades (di Giovanni et al., 2022). Our paper quantifies the output losses due to such shortages stemming from imbalances in sectoral supply and demand, conditional on higher prices. We develop a model in order to quantify the real GDP changes in a multi-country-multi-sector world, where countries are hit by Covid-19 related sectoral supply and demand shocks. These sectoral shocks can only be eliminated via global vaccinations in our model. Under unequal pace of vaccinations across the world, the sectoral shocks travel via supply chains from unvaccinated to vaccinated countries, allowing us to quantify the associated real output losses. We estimate the global and localized output gains from a counterfactual-more equal-vaccine distribution. ${ }^{2}$

Covid-19 is a "once in a life-time" shock for the global economy. It constitutes a series of disaggregated demand and supply shocks, affecting different countries at different times. While all countries are impacted by the virus, the effects are not synchronized across borders, unlike the 2008-09 Global Financial Crisis. The asynchronous effects of Covid-19 are due to the repeated nature of the health shock from different variants, and the unco-

\footnotetext{
${ }^{1}$ https://www.reuters.com/article/us-health-coronavirus-taiwan-idINKBN29X11P.

${ }^{2}$ As of August 2022, a third of the world is still not vaccinated, see, https://ourworldindata.org/covidvaccinations.
} 
ordinated health and economic policy responses across countries. The uneven vaccination policies combined with different degrees of aggregate demand-stimulus in different countries created sectoral demand and supply imbalances, known as global supply chain bottlenecks. $^{3}$

The real GDP losses of the vaccinated advanced economies (AEs), relative to their prepandemic levels, are estimated to be in the range of 0.1 to 1 percent. This range depends not only on the heterogeneity of the vaccine roll-out, but also on the elasticity of substitution between traded and domestic production inputs and consumption goods. In a scenario that mimics real life vaccine roll-out and uses short-run elasticities estimated in the literature, we show that vaccinated AEs lose around 0.6 percent of their pre-pandemic real GDP. This real GDP loss implies a rate of return of 260 percent for AEs from investing in global initiative COVAX that was set up to deliver vaccines to the emerging markets and developing economies (EMDEs). ${ }^{4}$

Our framework is based on Baqaee and Farhi (2022) which models disaggregated sectoral demand and supply shocks propagating through supply chains. As in Baqaee and Farhi (2022), these sectoral shocks create distinct cyclical conditions in different sectors. Our modeling contribution is to carry their structure to an open economy framework to capture such imbalances through global supply chains. The key contribution of our paper is empirical. Our multi-country model with full global input-output linkages allows us to quantify the losses for vaccinated countries associated with lack of vaccinations in other countries. Sectoral shocks associated with zero-Covid strategies of unvaccinated trading partners act as 'new' sectoral shocks in vaccinated countries. This is reminiscent to Guerrieri et al. (2022), where supply shock in one sector shows up as demand shock in another sector.

We measure the negative shocks to labor supply in a given sector-country pair with infections in that sector and lockdowns in that country. We assume labor is sector specific and not mobile across sectors. ${ }^{5}$ We allow for changes in labor demand as a result of changes

\footnotetext{
${ }^{3}$ At the time of our initial draft in January 2021, such disruptions were deemed to be temporary and predicted to be smoothed out before mid-2021 upon arrival of vaccines by the end of 2020. See, for example IMF World Economic Outlook (IMF, 2020). Our initial draft as well as the current draft delivers endogenous persistence of supply chain disruptions as long as the pandemic-related "sectoral" shocks keep hitting different country-industry pairs at different times.

${ }^{4}$ We arrived at the 260 percent estimate by dividing our calculated losses of 460 billion USD to 190 billion USD. The latter value is a rough estimate to manufacture enough vaccines to inoculate the world population. This number is projected from COVAX's estimated cost of 38 billion USD in early 2021 to manufacture enough doses of vaccines to vaccinate the vulnerable population.

${ }^{5}$ As shown by Fernald and $\mathrm{Li}$ (2022), labor re-allocation across sectors did not happen in the U.S. during the pandemic.
} 
in consumer demand, say from services to goods. Absent any nominal rigidity, sectoral wages adjust to establish equilibrium in a given sector, where immobile labor keeps labor market segmented across sectors.

Our two-period model is calibrated as follows. The first period is the pre-Covid-19 equilibrium and the second period is the pandemic, where we feed the sectoral shocks into the model. The length of the second period in the calibration determines when the economy goes back to pre-Covid-19 equilibrium and thus the persistence of the supply chain bottlenecks. To capture such granularity and heterogeneity across countries, we divide the second period into 365 days and solve the model daily. We feed in daily sectoral shocks as a function of daily disease dynamics, which are estimated based on the epidemiological model with starting parameters at the onset of the vaccine discovery. Our model features endogenous lockdowns. Labor supply in all non-essential sectors declines endogenously when the number of sick people exceeds a level that would fill a country's ICU bed capacity. We calibrate this threshold from the data. Lack of vaccinations in EMDEs make lockdowns more likely. If a country is fully vaccinated by a given day, we stop feeding-in the sectoral shocks and return to the equilibrium before Covid-19. Thus, the end of the second period is dictated by the vaccination scenarios and endogenous lockdowns in our calibrations.

Our novel identification of sector-level shocks depends on measuring sectoral infections using an epidemiological framework that allows us to convert country-level infections into sector-level. For this conversion, we start with pre-pandemic equilibrium values of employment in a given sector-country pair. We then shock each pair with infection-based demand and supply shocks to quantify the deviation from the equilibrium employment. The labor supply shock depends on the contact intensity of the sector, availability of remote work and lockdowns. In order to capture the compositional change in consumption from goods to services, we model a sectoral demand shifter. To calibrate the sectoral shifts, we use data on sectoral personal consumption expenditures available for the U.S. economy. Using this data, we fit the sectoral changes in consumption as a function of sectoral infections for the U.S. economy. We then project them to the remaining 64 countries using parameters from this exercise and country specific sectoral infections.

We use pre-pandemic global network data as the starting point of our calibration, before the sectoral shocks hit. The data comes from the OECD's latest available Inter-Country Input-Output (ICIO) tables from 2018 as shown in Figure 1a. Out of 65 countries, 39 countries are classified as AEs who have access to vaccines as early as January 2021. The remaining countries (including a residual entity called the "Rest of the World") belong to the set of 
EMDEs who are assumed to be unvaccinated at the onset. These international linkages are comprised of sectoral links. Sectors use inputs from a variety of other sectors as shown in Figure $1 \mathrm{~b} .{ }^{6}$ Once the sectoral shocks hit, each sector's expenditure on other sectors change. The model endogenously solves for these network changes via Domar weights and factor shares. As of the time of this writing, OECD's global network data is not updated to verify how the global trade network changed during the pandemic. There is anecdotal evidence on shipping delays and inventory adjustments during 2021. However, this is far from being sufficient to calculate endogenous changes in global network's expenditure shares, both at intensive and extensive margins. The evidence is thus insufficient to determine whether the firms changed their suppliers and/or how much they buy/sell to/from certain suppliers. Therefore, we provide a range of estimates based on different elasticities of substitution among the global network.

In the baseline calibration, we use low elasticities as estimated by Boehm et al. (2019, forthcoming) to capture the "just-in-time" nature of the global value chains. For robustness, we use higher elasticities as estimated by Caliendo and Parro (2015) that capture the substitution in the medium run. When the countries cannot easily substitute away their imported intermediate inputs from one country to another country in the short-run, the real GDP losses increase, more so for EMDEs. The effect of the degree of integration into trade and production network on the output losses is not trivial because there is a non-linear interaction between openness and elasticises of substitution. An open economy might be more integrated to global production network and hence has higher exposure to shocks. But an open economy might also have many suppliers. Hence, an open economy can also smooth the shocks she is exposed to if she can switch easily between the suppliers. Therefore, conditional on being hit by the same shock, an open economy will do better than a less open economy as the latter operates with few suppliers. This is why, under a low degree of substitution, the real GDP losses increase more for unvaccinated EMDEs who operate with less diversified supply chains (less open, few suppliers), a double whammy effect. Consistently, vaccinated AEs bear a higher share of the total world output loss under a high degree of substitution because losses of EMDEs are relatively lower than that of AEs.

\footnotetext{
${ }^{6}$ According to OECD (2020), the total value of world trade was 18 trillion USD. Within this total, intermediate products constituted 10.6 trillion USD, corresponding to 59 percent of world trade.
} 
Figure 1: Inter-country Inter-industry Trade Linkages

(a) International Trade Linkages

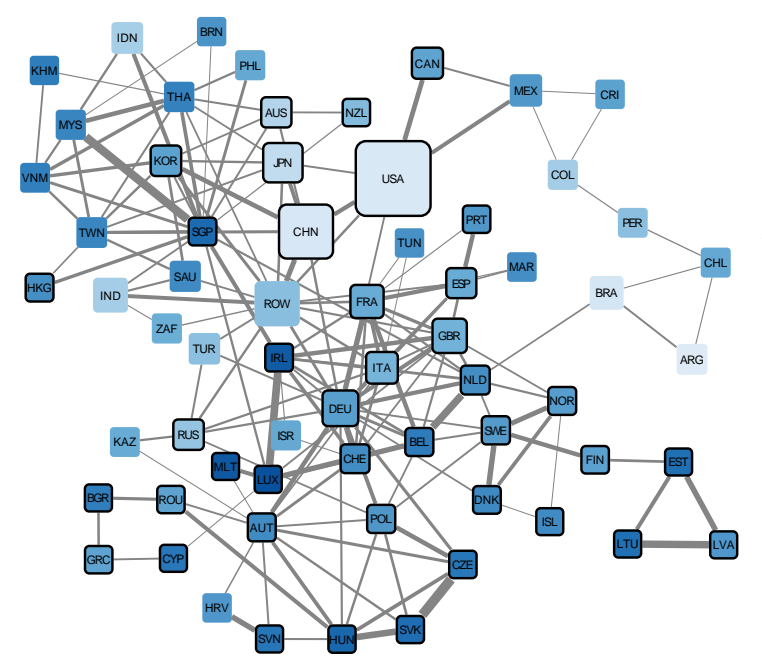

(b) Inter-industry Trade Linkages

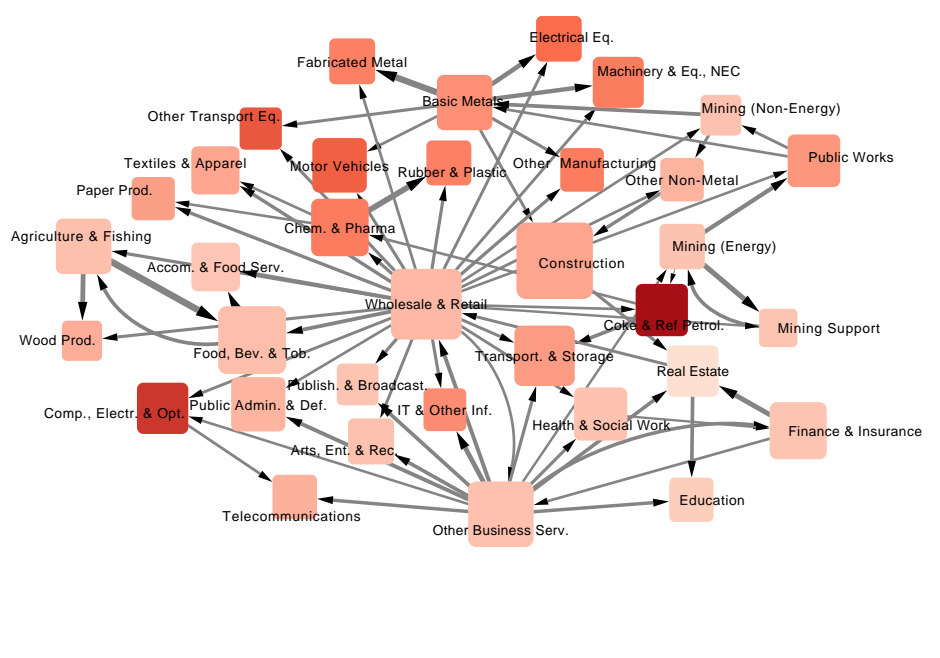

NOTES: Panel (a) shows a summary of international linkages. Each node corresponds to a country, with the node sizes proportional to the GDP of the country. The node color represents the openness of the country where openness is defined as the ratio of imports and exports to GDP. Darker shades represent more openness to trade (See Table C.1). Economies that have access to vaccines are denoted by black borders. The line between nodes gets thicker as bilateral trade increases. In total, there are 65 nodes and 168 lines shown on the network. Panel (b) shows aggregated inter-industry linkages. Each node corresponds to an industry. The node size represents the total intermediate usage of the industry. The smallest node corresponds to 184 billion USD for Mining Support industry and the largest node corresponds to 5.9 trillion USD in Construction industry. The node color represents the share of imported inputs in the industry. The lightest shade represents $5.9 \%$ in Real Estate industry and the darkest shade represents $37 \%$ in the Coke \& Refined Petroleum industry. The thickness of the directed lines from supply to target industry show the strength of the trade based on: (i) the intermediate input from the supply industry constitutes at least 10 percent of the inputs of target industry; or (ii) the supply industry is among the top two suppliers of the target industry. In total, there are 35 nodes and 72 lines shown on the network. Source: ICIO Tables OECD (2020).

The remainder of this paper is organized as follows. In Section 2, we present the model. In Section 3, we describe the data and the parameters for calibration. In Section 4, we report results of our quantitative analysis including robustness. Section 5 concludes.

\section{The Model}

Our model is set up to quantify real GDP changes in a multi-country-multi-sector world, where countries are hit by sectoral supply and demand shocks. We refrain from aggregate shocks and nominal rigidities on purpose. We capture the compositional changes in consumption demand together with labor supply shocks, in the absence of aggregate demand 
and / or productivity shocks. Prices and wages adjust fully and endogenously in response to sectoral shocks. The pandemic-related sectoral shocks can only be eliminated via vaccinations in our model. Under inequitable pace of vaccinations across the world, these sectoral shocks travel via supply chains from unvaccinated to vaccinated countries, allowing us to quantify the associated real output losses.

Our modeling of dis-aggregated sectoral demand and supply shocks propagating through supply chains and creating distinct cyclical conditions in different sectors is similar to Baqaee and Farhi (2022). Our contribution is carrying their structure to open economy framework to capture such imbalances through global supply chains. Our model shares the open economy focus with trade-network model of Baqaee and Farhi (2019), where the authors focus on aggregate effects of trade cost shocks. ${ }^{7}$ Our multi-country model with inter-sectoral linkages also allows us to quantify the losses of rich countries associated with the zero-Covid strategy of the Asian countries. Severe lockdowns in trading partners act as additional sectoral supply shocks in vaccinated countries.

We solve the analytical model by small perturbations/shocks that deliver an exact hatalgebra solution instead of solving the model by log-linearization around the steady state, similar to Baqaee and Farhi (2019). By integrating the response to many successive small shocks, the model can address relatively large shocks as well. Our computational implementation is fast and does not require extreme computational resources as in the case of trade-network models focusing on exact solutions. Compared to Baqaee and Farhi (2019), our solution is also faster, although the shocks we analyze are very different in nature. ${ }^{8}$

Our two period model has both an intratemporal and intertemporal dimension. Within a given period, the intratemporal trade matters. Across the two periods, the consumption adjusts to demand and supply shocks. Because there is no aggregate demand shock (or discount factor shock), the intertemporal side becomes irrelevant in terms of shifting consumption between two periods. The first period is the pre-pandemic year of 2019 and the second period is the pandemic period (2020-2021), where all sectoral shocks take place. Hence, all shocks are unanticipated and render consumers as hand-to-mouth agents consuming their current income in each period.

\footnotetext{
${ }^{7}$ We abstain from that as there were no significant tariff changes by sector during the last two years. Defacto trade barriers observed were an endogenous outcome of pandemic related shocks and policies such as lockdowns.

${ }^{8}$ Our difference from Baqaee and Farhi (2019) is that we hard code all different types of Allen-Uzawa elasticities in our computations, reducing the time needed for full solution in each perturbation.
} 


\subsection{Environment}

Notation. Each country produces and consumes final and intermediate goods and services. These goods and services are produced with factors present in each country, and traded with each other. We denote the set of countries with $\mathcal{C}$ and we index countries with $c, v$ or $m$. The $\mathcal{N}$ denotes the set of industries or sectors (we use these terms interchangeably) which are indexed by $i, j$ or $k$. A sector $i$ in country $c$ is denoted by $i c$ and we denote set of all such pairs with $\mathcal{C N}$. Production in ic can use inputs from sector $j$ in country $m$, i.e., $j m$. For example automotive industry, $i$, in Germany, $c$, imports steel, $j$, from country $m$, Turkey. For convenience, we also introduce the consumption as sector $0 \notin \mathcal{N}$ and consumption in country $c$ is indexed by $0 c$. The set of factors is represented by $\mathcal{F}$ and indexed by $f$ or $g$. Because we work with more than 2 countries, more than 2 sectors, and more than 2 factors, this notation is essential. We denote the set of factors present and owned by country $c$ with $\mathcal{F}_{c}$.

Prices, outputs and expenditures. Suppose the output of industry $i$ in country $c$ is denoted with $y_{i c}$ and its price with $p_{i c}$. The country-sector pair $i c$ uses inputs from other sectors from different countries in addition to labor. We show the inputs used from sector $j$ in country $m$ by sector $i$ in country $c$ with $x_{j m}^{i c}$ and the price of this input is $p_{j m} \cdot{ }^{9}$

Recall that $i=0$ denotes the consumption of households. We assume $y_{0 c}=C_{c}$ is the total consumption in country $c$ and the price index of consumption is denoted by $p_{0 c}$. Let's denote the total nominal expenditure of country $c$ with $E_{c}=p_{0 c} y_{0 c}=p_{0 c} C_{c}$. The total world expenditure is $E \equiv \sum_{c} E_{c}$. We assume that the factors are country and sector specific.

For any factor $f$ in country $c$, we denote the corresponding labor with $L_{f c}$ and its wage with $w_{f c}$. We refer to the specific factor used in country-sector pair ic with $f_{i c}{ }^{10}$

Input-Output Matrix. We use expenditure shares as the building blocks of both consumption and the production sides of the economy given the network linkages. These shares constitute the input-output matrix. The input share of sector $j$ of country $m$ in sector $i$ in

\footnotetext{
${ }^{9}$ We solve the changes in the log prices and quantities to implement hat algebra used in the trade literature (Costinot and Rodríguez-Clare, 2014; Caliendo and Parro, 2015). We assume that the trade costs, specifically tariffs, do not change during the pandemic. The increase in shipping costs enters into the model because transportation sector is represented as a separate entry in the Input-Output matrix. In the model, we initialize the price of each good to be 1 in each country. Hence, when we solve for log-changes, implying price changes for a given variety is same in each country.

${ }^{10}$ We can extend our notation that we used for sectors above to factors with $x_{f_{c}}^{i c} \equiv L_{f c}$ and $p_{f_{c}} \equiv w_{f c}$. Since there is a single factor (labor), we also use $L_{i c}$ to denote the labor used in production of country-sector pair $i c$.
} 
country $c$ is defined by:

$$
\Omega_{j m}^{i c} \equiv \frac{p_{j m} x_{j m}^{i c}}{p_{i c} y_{i c}} .
$$

We express the expenditure share of industry $j m$ in final good consumption as:

$$
\Omega_{j m}^{0 c} \equiv \frac{p_{j m} x_{j m}^{0 c}}{E_{c}}
$$

where $x_{j m}^{0 c}$ denotes the amount of industry jm's output consumed by the households in country $c$. Finally, the value-added (or factor share) in country-sector pair $i c$ is defined as:

$$
\alpha_{i c} \equiv \Omega_{f_{i c}}^{i c} \equiv \frac{w_{f_{i c}} L_{f_{i c}}}{p_{i c} y_{i c}}=1-\sum_{j m \in \mathcal{C} \mathcal{N}} \Omega_{j m}^{i c} .
$$

Nominal Expenditure and Real GDP. In our open economy model, we assume that the trade is balanced. With this assumption, nominal GDP and GNE are equal to each other. Hence, we can write the expenditure of country $c$ in terms of the total factor income of the country:

$$
E_{c}=\sum_{f \in \mathcal{F}_{c}} w_{f} L_{f} .
$$

The real GDP of country $c$ is then denoted by $\mathrm{GDP}_{c}$ and calculated as:

$$
\mathrm{GDP}_{c}=\frac{E_{c}}{p_{0 c}},
$$

which is the total expenditure divided by the consumption price index.

\subsection{Production}

Figure 2 summarizes our production side. Production in each sector $i$ in country $c$ is achieved by combining sector-specific labor and a bundle of intermediate inputs. The assumption of sector-specific labor implies that labor is not mobile between sectors but can fluctuate within a sector over time. The empirical evidence during the pandemic supports the presence of within sector reallocation and the absence of between sector reallocation as shown by Fernald and Li (2022). The intermediate bundle for a sector-country pair ic consists of all the inputs from different sector bundles. These sector bundles, in turn, are 
Figure 2: Production Structure

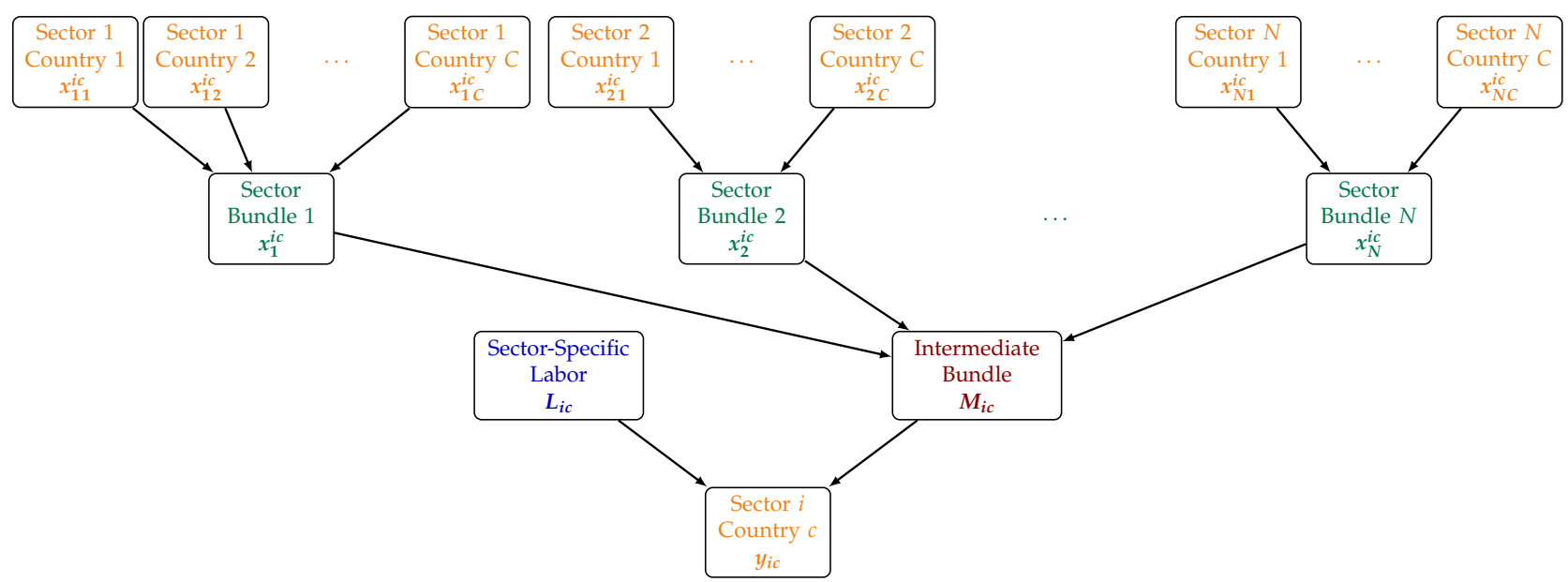

NOTES: This figure presents the inputs used in the production for sector $i$ in country $c$. All the aggregations are done with functions exhibiting constant elasticity of substitution, albeit with different elasticities. Sector varieties, sector bundles, intermediate bundles and labor are all country-sector specific. We show the notation that we use for each input in the last line of each box..

formed by different sector-country varieties.

Let's explain this production structure with an example from German automotive manufacturing. German automobiles are produced by German workers and an intermediate bundle. This intermediate bundle consists of sector bundles such as steel, plastic, textiles, leather, and glass. These sector bundles are aggregates of sector varieties from different countries. The steel bundle for German automotive manufacturing, for instance, is formed by steel from Turkey, India, China, the U.S., etc. We assume that the production follows this three-layer nested CES structure. Next, we formalize each of these steps shown in Figure 2 and highlight the key parameters.

The production in country-sector pair $i c$ is achieved by combining the sector specific labor and the intermediate bundle. We denote the price of this bundle with $p_{M}^{i c}$ and its quantity with $M^{i c}$. We assume that in the production of the final good, the production technology is constant elasticity of substitution (CES) with elasticity $\phi$. The normalized production function can be written as:

$$
y_{i c}=\frac{A_{i c}}{\bar{A}_{i c}}\left[\alpha_{i c}\left(\frac{L_{i c}}{\bar{L}_{i c}}\right)^{\frac{1-\phi}{\phi}}+\left(1-\alpha_{i c}\right)\left(\frac{M_{i c}}{\bar{M}_{i c}}\right)^{\frac{1-\phi}{\phi}}\right]^{\frac{\phi}{1-\phi}} .
$$

where the values with a bar on top denote the pre-pandemic 2019 equilibrium normaliza- 
tion and $A_{i c}$ is the productivity. ${ }^{11}$ The corresponding price index is:

$$
p_{i c}=\left[\alpha_{i c}\left(w_{f_{i c}}\right)^{1-\phi}+\left(1-\alpha_{i c}\right)\left(p_{M}^{i c}\right)^{1-\phi}\right]^{\frac{1}{1-\phi}} .
$$

We assume $0 \leq \phi<1$, i.e., labor and intermediate inputs are complements. As we show in the calibration section, this assumption is supported by the estimates we use from the empirical literature.

The price of the intermediate bundle, $p_{M^{\prime}}^{i c}$ can be written as a function of the price of the sector bundle of $j$ to be used in industry $i c$, which we denote by $p_{j}^{i c}$. Assuming a CES aggregator with elasticity of substitution of $\varepsilon$, we can write the price for the intermediate bundle as:

$$
p_{M}^{i c}=\left[\sum_{j \in \mathcal{N}} \frac{\Omega s_{j}^{i c}}{1-\alpha_{i c}}\left(p_{j}^{i c}\right)^{1-\varepsilon}\right]^{\frac{1}{1-\varepsilon}},
$$

with

$$
\Omega s_{j}^{i c} \equiv \sum_{m \in \mathcal{C}} \Omega_{j m}^{i c} .
$$

$\Omega s_{j}^{i c}$ captures the share of sector $j$ in production of $i c$ and is calculated by summing over the country varieties. We assume that $0 \leq \varepsilon<1$, i.e., all sectoral inputs for the intermediate bundle are complements. This assumption says that the plastic and steel cannot be easily substituted in the auto production, which is backed by the estimates from the empirical literature as we show in our calibration section.

Sector bundles are aggregates of varieties coming from different countries, with a price index:

$$
p_{j}^{i c}=\left[\sum_{m \in \mathcal{C}} \frac{\Omega_{j m}^{i c}}{\Omega s_{j}^{i c}}\left(p_{j m}\right)^{1-\tau_{j}}\right]^{\frac{1}{1-\widetilde{\zeta}_{j}}}
$$

using a CES aggregator with a sector-specific elasticity of substitution $\xi_{j}$. These sector specific elasticities are calculated to create substitutions between varieties coming from different countries in the long term (Costinot and Rodríguez-Clare, 2014; Caliendo and Parro, 2015) such that $\xi_{j} \geq 1$. But in the short term, we assume that it is hard to immediately substitute varieties from different countries to capture what happened during the pandemic. The case of $\xi_{j} \leq 1$ is estimated by Boehm et al. (2019) under earthquake shocks and captures the real-life supply chain bottlenecks associated with the pandemic. Our empirical exercise

\footnotetext{
${ }^{11}$ Here, for the model's completeness, we include the sectoral productivity term. But in our calculations, we do not introduce any productivity shocks.
} 
presents results for the cases of low and high substitution, for the range of $\xi_{j} \leq 1 \& \xi_{j} \geq 1$.

\subsection{Consumption}

There is a representative agent in each country solving a two-period consumption optimization problem. The first period corresponds to the pre-pandemic stage and the second period corresponds to the pandemic stage. In each country $c$, there is a continuum of households. These households collectively own all the primary factors and their source of income is the wage from the factors. The pandemic comes as a total surprise and effectively makes all our households live as hand-to-mouth households consuming what they earn in each period. Hence, in each period, the national expenditure is equal to: $E_{c, t}=\sum_{f \in \mathcal{F}_{c}} L_{f, t} w_{f, t}$. We normalize the world expenditure to 1 in both periods. Therefore, we drop the time-subscript below because we focus on the changes in the production and consumption patterns during the pandemic period.

Next, we turn to the within-period problem. Similar to our multi-layer nested production structure, we also assume a nested structure in consumption as depicted in Figure 3. We assume that a representative consumer in country $c$ enjoy goods and services at the

Figure 3: Consumption Structure

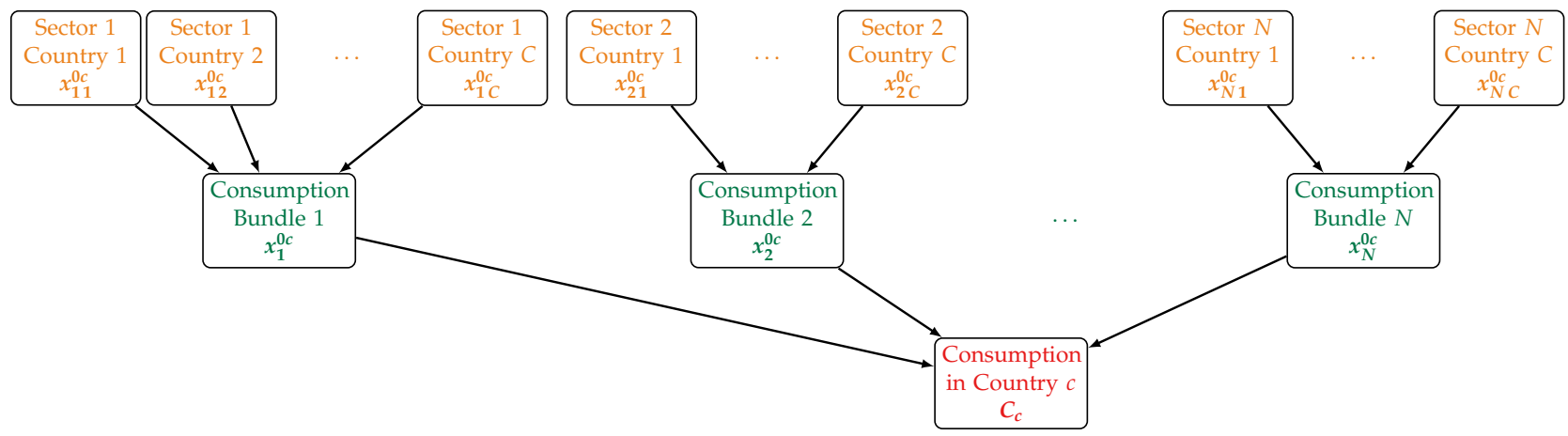

NOTES: This figure presents the consumption choice in country $c$. All the aggregations are done with functions exhibiting constant elasticity of substitution albeit with different elasticities. The consumption bundles are country specific.

sectoral consumption bundle levels. This amounts to optimizing their consumption to:

$$
C_{c}=\left[\sum_{j \in \mathcal{N}} \Omega s_{j}^{0 c}\left(x_{j}^{0 c}\right)^{\frac{\sigma-1}{\sigma}}\right]^{\frac{\sigma}{\sigma-1}},
$$


where $x_{j}^{0 c}$ denotes the (normalized) sectoral consumption and

$$
\Omega s_{j}^{0 c} \equiv \sum_{m \in \mathcal{C}} \Omega_{j m}^{0 c}
$$

denotes the share of industry $j$ in the final consumption of consumers in country $c$. We assume $\sigma$ to be equal to 1 to mimic a Cobb-Douglas preference structure. Similar to the production side, consumption bundles that are comprised of varieties from different countries are optimized by consumers among different country varieties with:

$$
x_{j}^{0 c}=\left[\sum_{m \in \mathcal{C}} \frac{\Omega_{j m}^{0 c}}{\Omega_{j}^{0 c}}\left(x_{j m}^{0 c}\right)^{\frac{\tilde{\xi}_{j}^{\prime}-1}{\bar{\xi}_{j}^{\prime}}}\right]^{\frac{\xi_{j}^{\prime}}{\bar{\xi}_{j}^{\prime}-1}} .
$$

The corresponding consumption price index can be written as:

$$
p_{0 c}=\left[\sum_{j \in \mathcal{N}} \Omega s_{j}^{0 c}\left(p_{j}^{0 c}\right)^{1-\sigma}\right]^{\frac{1}{1-\sigma}},
$$

where $p_{j}^{0 c}$ denotes the price of the consumption bundle $j$ in country $c, \sigma=1$ corresponds to the Cobb-Douglas case with constant expenditure shares, where the price index, in the limit, becomes:

$$
\log p_{0 c}=\sum_{j \in \mathcal{N}} \Omega s_{j}^{0 c} \log p_{j}^{0 c} .
$$

The price equation for consumption bundle $i$ of country $c$ is:

$$
p_{j}^{0 c}=\left[\sum_{m \in \mathcal{C}} \frac{\Omega_{j m}^{0 c}}{\Omega_{j}^{0 c}}\left(p_{j m}\right)^{1-\xi_{j}^{\prime}}\right]^{\frac{1}{1-\xi_{j}^{\prime}}} .
$$

As in the production side, $\xi_{i}^{\prime}$ is the trade elasticity (consumption side), usually taken from Caliendo and Parro (2015) as greater than or equal to one. In our baseline analysis, we use the same $\xi_{i}^{\prime}$ elasticity as sector bundles in the production side consistent with Boehm et al. (forthcoming), but we also study the cases where the elasticities are markedly different from each other. 


\subsection{Equilibrium and Perturbation}

Given the parameters, a general equilibrium consists of good prices, factor prices, outputs, intermediate inputs, factor inputs, and consumption levels such that producers minimize their costs, households maximize their utilities, and good and factor markets clear. The equilibrium is stable in the absence of any shocks. We perturb this equilibrium with sectoral demand and supply shocks. At each point in time, we assume that:

- Good markets clear such that for any industry ic:

$$
y_{i c}=\sum_{m \in \mathcal{C}} \sum_{j \in \mathcal{N}} x_{i c}^{j m}+\sum_{m \in \mathcal{C}} x_{i c}^{0 m} .
$$

The first term corresponds to the intermediate input usage of $i c$ in all country-industry pairs, indexed by $j m$, and the second term corresponds to the final good usage of $i c$ in all countries, indexed by $m$.

- Labor markets clear: all "potential" sector-specific workers are employed.

Initially, we set all prices to 1 and all output of country-sector pairs to their respective share in the total nominal world expenditure. The model is calibrated in such a way that this is the equilibrium. After perturbing with shocks, the prices and outputs will re-equilibriate. Next, we explain how we model pandemic related shocks.

\subsection{Modeling Pandemic Related Shocks}

\subsubsection{Nature of the Shocks}

Covid-19 pandemic affected supply and demand. Importantly, both supply and demand shocks are sector-specific. To model pandemic related sectoral shocks, we start with shocks to "potential" labor supply. People may not necessarily die, but they still cannot go to work either because they are sick, or taking care of sick, or their workplace is not safe, or their employers operate under other restrictions. All these factors will have negative effects on labor supply, making the economy to operate inside the production possibilities frontier.

There is extensive survey-based evidence from the U.S. that Covid-19 is a key reason for not going to work, during 2021, as shown in Figure 4a below. Correlation between Covid19 cases and the number of people not going to work due to self-reported pandemic related reasons is 84 percent from July 2020 to July 2022 based on weekly data. Figure 4b shows 
that labor supply-linked to health-in the U.S. has not recovered to pre-pandemic levels. As shown, the average number of people absent from work due to illness is significantly higher during the pandemic compared to pre-pandemic period.

Figure 4: The Effects of Covid-19 on U.S. Labor Supply

(a) Not working due to Covid-19

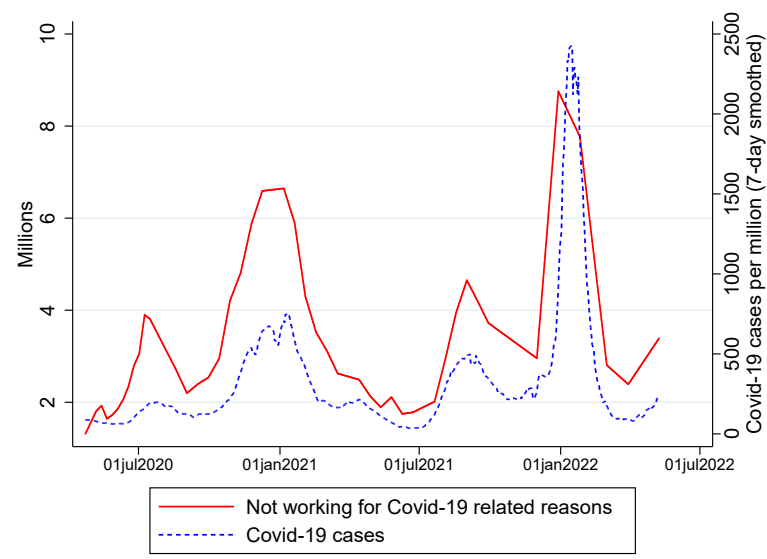

(b) Not working due to own illness over time

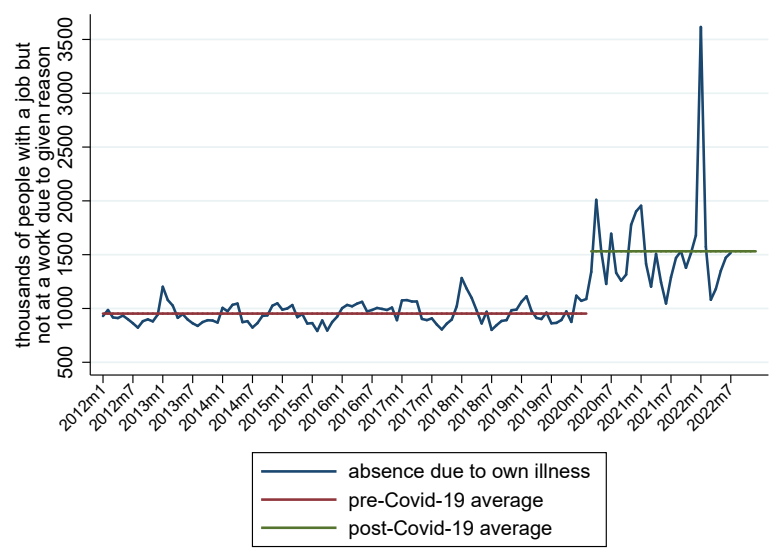

NOTES: (a) This panel plots the co-movement of the weekly number of people who reported that they cannot work due to "pandemic-related reasons" with the weekly number of Covid-19 cases. See Section A.1.1 for the details on the construction of the series obtained from the U.S. Census Bureau Household Pulse Survey. (b) This panel plots the number of employed people in the U.S. labor market who are absent in a given month. The sample period is from January 2012 to July 2022. The U.S. Bureau of Labor Statistics conducts Current Population Survey which provides a monthly measure that tracks the number of employed people who are absent from work due to own illness, injury or medical problems. See Figure A.3 for details in Appendix A.1.2.

Figure 5 illustrates how our model incorporates such labor supply shocks. We denote the equilibrium labor with $L_{f}$. As the pandemic progresses, sectoral labor supply initially declines due to sickness, shown with $L_{f}$ with blue line. During lockdowns, for a nonessential sector, only people in teleworkable jobs can work, so that labor supply is capped with the dotted blue line. During the recovery phase, depending on vaccinations, sectoral demand shifts, and the share of teleworkable jobs, labor can go up and down but never reaches the pre-pandemic full employment level, because the initial shock to labor supply never eases due to the absence of global vaccinations. Notice that the sectoral heterogeneity and segmented labor markets are important here. There can be sectors where labor supply can go back to the pre-pandemic level. However, as long as there are other sectors where this does not happen, global labor supply will not recover. This is consistent with the coexistence of a labor force participation rate that is below the pre-pandemic level and tight labor markets in many countries. 
On the consumption side, it is well known that sectoral consumption shifted from services to goods in the early phase of the pandemic and started shifting back slowly during the recovery phase (e.g see Figure C.5a for the U.S.). di Giovanni et al. (2022) documented different timing of such compositional changes in consumption across several countries. Chetty et al. (2020) emphasize that the fear of contacting the disease as the main source of the decline in spending at the initial stages of the pandemic in contact-intensive sectors. Similarly, using cell phone data to track movements of individuals, Goolsbee and Syverson (2021) show that even though the consumer traffic fell by $60 \%$, only $7 \%$ could be explained by the shutdown restrictions. The authors suggest that the changes in consumer behavior are most likely driven by the fear of infection. We model this as a sectoral demand shifter.

Figure 5: Labor during Pandemic

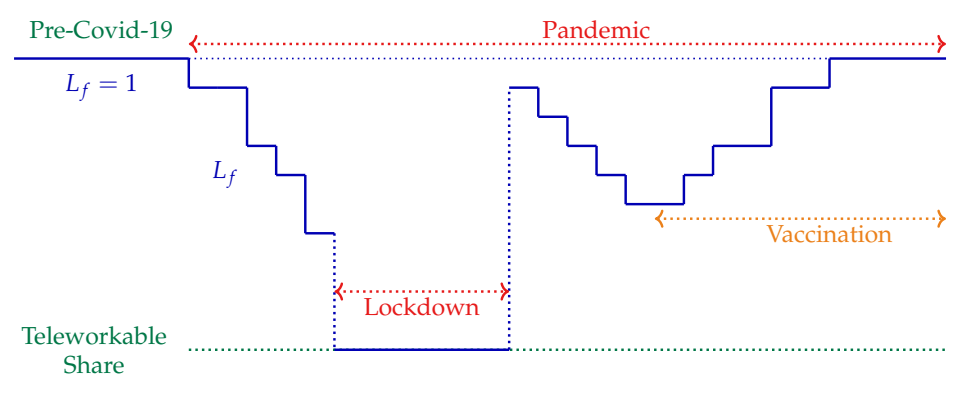

To connect the modeling of pandemic related sectoral consumption demand and labor supply shocks to infections, we use an epidemiological framework. Figure 6 summarizes how we combine our model with an epidemiological framework. The bottom half of the figure describes the supply side and the top half depicts the demand side. On the supply side, the transmission dynamics of the virus would differ depending on whether the workers are on-site or at a remote location like home. Among the professions that need to be carried out on the work site, we assume that the viral transmission depends on the physical proximity between the workers or between the workers and the customers. An on-site worker could be exposed to infection either at work or outside work. Intermediate inputs, including the imported ones, directly affect supply. Production of domestic inputs are affected from domestic labor supply shocks linked to infections. Imported inputs are affected from the evolution of the pandemic in the other countries. On the demand side, both domestic and foreign demand for final and intermediate goods change with consumer preferences depending on the infection levels at home and foreign countries. 
Figure 6: Modelling the effects of the Covid-19 shock in a multi-sector open economy

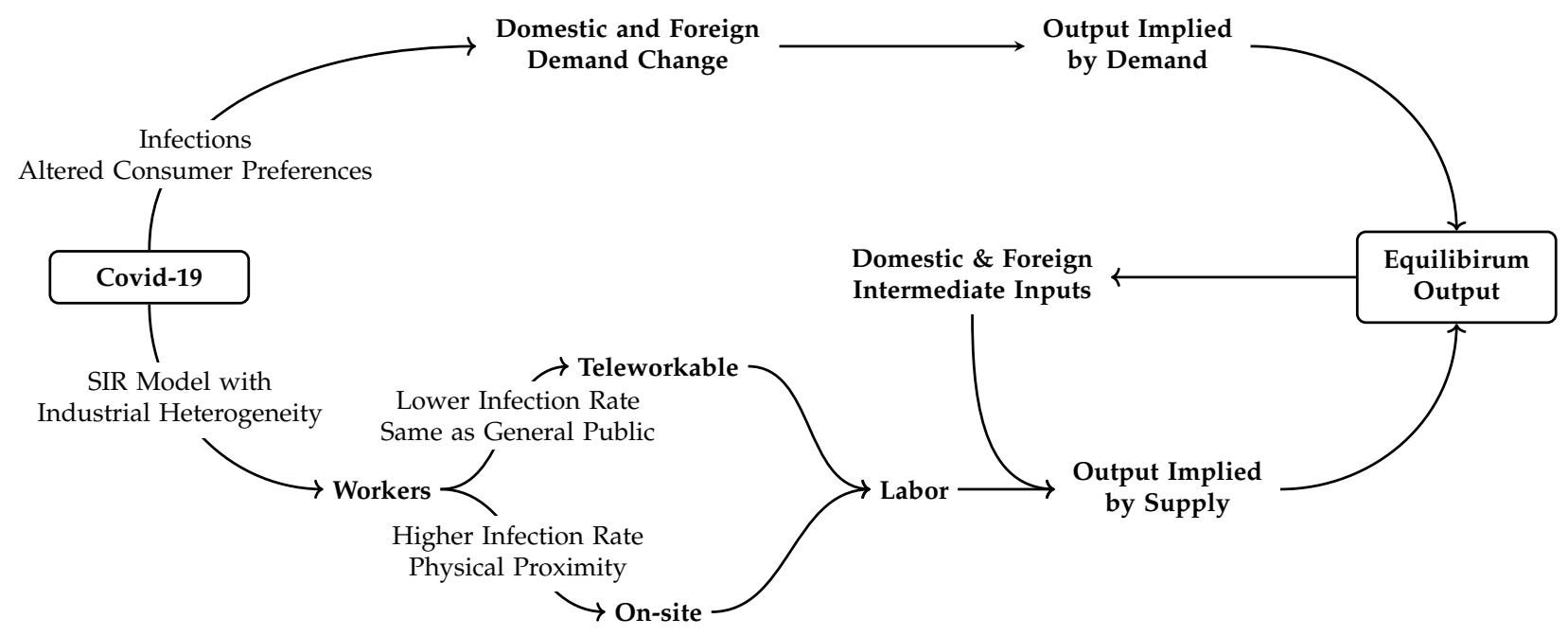

\subsubsection{The Epidemiological SIR Model}

We use the workhorse framework in many epidemiological studies, the Susceptible-InfectedRecovered (SIR) model. ${ }^{12}$ Let's take a population of size $N$. At any given time $t$, we can split the population into three classes of people: Susceptible $\left(S_{t}\right)$, Infected $\left(I_{t}\right)$ and Recovered $\left(R_{t}\right)$. We provide the model for a single country, suppressing the country index for the ease of the demonstration. ${ }^{13}$ The susceptible group does not yet have immunity to the disease, and the individuals in this group have the possibility of getting infected. The recovered group, on the other hand, consists of individuals who are immune to the disease. Immunity can be developed either because the individual goes through the infection or because they get vaccinated. The SIR model builds on the simple principle that a fraction of the infected individuals in the population, $\frac{I_{t-1}}{N}$, can transmit the disease to susceptible ones $S_{t-1}$ with an (structural) infection rate of $\beta$. Therefore, the number of newly infected individuals in the current period is $\beta S_{t-1} \frac{I_{t-1}}{N}$. The newly infected individuals should be deducted from the pool of susceptible individuals in the current period. Meanwhile, in each period, a fraction $\gamma$ of the infected people recover from the disease, which in turn reduces the number of actively infected individuals. To track any changes in the number of individuals in the above-mentioned three groups, the following set of difference equations are used:

$$
\Delta S_{t}=-\beta S_{t-1} \frac{I_{t-1}}{N}
$$

\footnotetext{
${ }^{12}$ See for example Allen (2017) among others.

${ }^{13}$ We do not model cross-country infections due to travel as a source of prolonged pandemics.
} 


$$
\begin{aligned}
\Delta R_{t} & =\gamma I_{t-1} \\
\Delta I_{t} & =\beta S_{t-1} \frac{I_{t-1}}{N}-\gamma I_{t-1}
\end{aligned}
$$

The law of motion for the number of infected individuals shows the trajectory of the pandemic at the aggregate level. Note that, $\Delta S_{t}+\Delta R_{t}+\Delta I_{t}=0$ holds at any given time, assuming that the size of the population remains constant. ${ }^{14}$

We modify the canonical SIR model to allow for sectoral heterogeneity in terms of the size and working conditions that can lead to distinct infection trajectories in each sector. The transmission of the virus accelerates with close physical proximity. Hence, employees working in the industries with higher physical proximity are infected with a higher probability. We assume that the economy is composed of $K$ sectors. We denote the industries by subscript $i=1, \ldots, K$. Each industry has $L_{i}$ workers (since factors are industry specific, we can index them with industry index) and there is also the non-working population which we denote by $N_{N W}$. Each industry has two types of workers: (i) employees who can perform their jobs remotely (i.e., teleworkable) and (ii) employees who need to be on-site to fulfill their tasks. In each industry, we denote the number of employees in the first group with $T W_{i}$ and the second group with $N_{i}$. Hence:

$$
L_{i}=T W_{i}+N_{i}
$$

For the disease propagation, we lump the non-working population and the employees in the teleworkable jobs together, and call them the "at-home" group. We denote the at-home group with index $i=0$. The total number of individuals in this group is, therefore:

$$
N_{0}=N_{N W}+\sum_{i=1}^{K} T W_{i}
$$

Suppose that the infection rate in the at-home group is $\beta_{0}$. In order to account for heterogeneous physical proximities across industries, we compute the rate of infection for each industry $i$, denoted by $\beta_{i}$, as:

$$
\beta_{i}=\beta_{0} \operatorname{Prox}_{i} \quad \text { for } \quad i=1, \ldots, K
$$

\footnotetext{
${ }^{14} \mathrm{~A}$ small fraction of the resolved cases includes deaths due to disease, reducing the population size. In our setting, we suppress the fraction of death cases in the recovery rate parameter $\gamma$, assuming that the population remains fixed for the SIR model to remain tractable. Therefore, throughout the text, we use the terms "resolution rate" and the "recovery rate" interchangeably for the parameter $\gamma$. See also Atkeson (2020), Bendavid and Bhattacharya (2020), Dewatripont et al. (2020), Fauci et al. (2020), Li et al. (2020), and Vogel (2020) on different estimates of recovery and death rates.
} 
where $\operatorname{Prox}_{i}$ is the proximity index for industry $i$ that captures the contact intensive nature of the industry.

Here, $S_{i, t}, I_{i, t}$ and $R_{i, t}$ denote the number of susceptible, infected and recovered individuals, respectively, and $N_{i}=S_{i, t}+I_{i, t}+R_{i, t}$ denotes the total number of on-site individuals in industry $i$ and the at-home group $(i=0)$. Susceptible individuals in the at-home group can get infected from the infected individuals in the entire society:

$$
\Delta S_{0, t}=-\beta_{0} S_{0, t-1} \frac{I_{t-1}}{N}
$$

where $I_{t}=\sum_{i=1}^{K} I_{i, t}+I_{0, t}$ captures the total number of infected individuals. An on-site worker in sector $i$, however, could be exposed to infection either at work, at the rate of $\beta_{i} S_{i, t-1} \frac{I_{i, t-1}}{N_{i}}$, or outside work, that involves all the remaining activities -including family life, shopping and commuting- at the rate $\beta_{0} S_{i, t-1} \frac{I_{t-1}}{N}$. Hence, the number of susceptible individuals among the on-site workers in industry $i$ changes as:

$$
\Delta S_{i, t}=-\beta_{i} S_{i, t-1} \frac{I_{i, t-1}}{N_{i}}-\beta_{0} S_{i, t-1} \frac{I_{t-1}}{N} .
$$

The recovery rate is the same for all types of infected individuals:

$$
\Delta R_{i, t}=\gamma I_{i, t-1}
$$

The number of infected individuals changes as the susceptible individuals get infected and some infected individuals recover from the disease:

$$
\Delta I_{i, t}=-\left(\Delta R_{i, t}+\Delta S_{i, t}\right)
$$

With industrial heterogeneity, we match the employment size weighted average $\beta_{i}{ }^{\prime}$ s of the infected individuals to observed overall $\beta$ in a country. For an on-site worker in industry $i$, the implied $\beta$ parameter can be approximated by $\left(\beta_{0}+\beta_{i}\right)$. For a non-working individual, this parameter is only $\beta_{0}$. Using equation (11), we impose:

$$
\beta_{0} \frac{N_{0}}{N}+\sum_{i=1}^{K}\left(\beta_{0}+\beta_{i}\right) \frac{N_{i}}{N}=\beta_{0}+\beta_{0} \sum_{i=1}^{K} \operatorname{Prox}_{i} \frac{N_{i}}{N}=\beta
$$


Hence, we solve for $\beta_{0}$ in terms of $\beta$, industry size, and the proximity levels as:

$$
\beta_{0}=\beta\left(1+\sum_{i=1}^{K} \frac{\operatorname{Prox}_{i} N_{i}}{N}\right)^{-1} .
$$

Once the parameters are computed, the evolution of infections in the extended multi-sector SIR model can be written as:

$$
\Delta \mathcal{I}_{t}=F \mathcal{I}_{t-1}-\gamma \mathbb{I}_{\mathrm{K}} \mathcal{I}_{t-1}
$$

where $\mathcal{I}_{t}=\left(I_{0, t}, I_{1, t}, \ldots, I_{i, t}, \ldots, I_{K, t}\right)^{\prime}$ and $\mathbb{I}_{\mathbb{K}}$ is the $K$ dimensional identity matrix. $F$ matrix is defined as:

$$
F=\left[\begin{array}{cccccc}
\beta_{0} \frac{S_{0, t-1}}{N} & \beta_{0} \frac{S_{0, t-1}}{N} & \ldots & \ldots & \beta_{0} \frac{S_{0, t-1}}{N} & \beta_{0} \frac{S_{0, t-1}}{N} \\
\beta_{0} \frac{S_{1, t-1}}{N} & \beta_{0} \frac{S_{1, t-1}}{N}+\beta_{1} \frac{S_{1, t-1}}{N} & \beta_{0} \frac{S_{1, t-1}}{N} & \ldots & \ldots & \beta_{0} \frac{S_{1, t-1}}{N} \\
\beta_{0} \frac{S_{2, t-1}}{N} & \beta_{0} \frac{S_{2, t-1}}{N} & \beta_{0} \frac{S_{2, t-1}}{N}+\beta_{2} \frac{S_{2, t-1}}{N} & \beta_{0} \frac{S_{2, t-1}}{N} & \ldots & \beta_{0} \frac{S_{2, t-1}}{N} \\
\vdots & \vdots & & \ddots & & \vdots \\
\vdots & \vdots & & & \ddots & \vdots \\
\beta_{0} \frac{S_{K, t-1}}{N} & \beta_{0} \frac{S_{K, t-1}}{N} & \ldots & \ldots & & \beta_{0} \frac{S_{K, t-1}}{N}+\beta_{K} \frac{S_{K, t-1}}{N_{K}}
\end{array}\right]
$$

Using these system matrices, $R_{0}$ can be computed using the largest eigenvalue of the matrix $F^{-1} v$. Given the initial size of the groups based on employment numbers, the eigenvalue would approximately correspond to the normalization present in equation (12).

\subsubsection{Sectoral Infections and Production}

Sectors are heterogeneous in terms of the share of teleworkable jobs and physical proximity requirements, which results in differential sector specific labor supply shocks during the pandemic. Once the virus hits and spreads among the workers, the total number of workers in a given country-sector pair $i c$ changes to $L_{i c, t}$ as a function of the infections and can be written as:

$$
L_{i c, t}=\left(N_{i c}-I_{i c, t}\right)+T W_{i c}\left(1-\frac{I_{0 c, t}}{N_{0 c}}\right)=L_{i c}-I_{i c, t}-T W_{i c} \frac{I_{0 c, t}}{N_{0 c}}
$$

where $N_{i c}$ is the number of workers in the on-site group in industry-country pair $i c, I_{i c, t}$ is the number of infected workers in the on-site group, and $T W_{i c}$ is the number of workers in the at-home group (i.e., those who can work remotely) in industry-country pair $i c$. The ratio 
$I_{0 c, t} / N_{0 c}$ captures the fraction of infected individuals in the at-home group, which includes the non-working population as well as all workers in the at-home group (i.e., teleworkers) in country $c$.

The sector specific shock for industry ic is a function of the number of infected workers in the on-site group, the share of teleworkable jobs in sector $i$ and lockdowns in country $c$. Let us denote the vector of all infections in the country with $I_{c, t} \cdot{ }^{15}$ The sectoral labor shock can be expressed as:

$$
\Delta_{i c}\left(I_{c, t}\right)= \begin{cases}\frac{L_{i c}-I_{i c, t}-T W_{i c} \frac{I_{0 c, t}}{L_{i c}}}{L_{i c}} & \text { if } c \text { is not under lockdown or } i \text { is essential, } \\ \frac{T W_{i c}-T W_{i c} \frac{I_{0, t}}{N_{0 c}}}{L_{i c}} & \text { if } c \text { is under lockdown and } i \text { is non-essential. }\end{cases}
$$

The lockdown decision is endogenous and triggered when the total active infected cases passes a threshold, i.e., $\sum_{i} I_{i c, t} \leq \rho$. The parameter $\rho$ is calibrated to the ratio of infected people to intensive care units (ICUs). Note that individuals employed in the essential sectors continue to work even under lockdown. The production in the given country-sector pair ic changes as a response to the labor supply shock specific to that pair. It can be expressed as follows:

$$
y_{i c, t}=\frac{A_{i c}}{\bar{A}_{i c}}\left[\alpha_{i c}\left(\frac{\Delta_{i c}\left(I_{c, t}\right) L_{i c}}{\bar{L}_{i c}}\right)^{\frac{1-\phi}{\phi}}+\left(1-\alpha_{i c}\right)\left(\frac{M_{i c}}{\bar{M}_{i c}}\right)^{\frac{1-\phi}{\phi}}\right]^{\frac{\phi}{1-\phi}} .
$$

\subsubsection{Sectoral Infections and Consumption}

As we relate the sectoral demand changes to infection levels, we obtain sectoral demand shocks for each country. We show the details of our calculations in Section 3.2. In our setting, we modify the pricing of the sectoral consumption bundle described in equation (6) to allow for a sector specific demand shifter, denoted as $\delta_{j}^{0 c}$. The demand shifter evolves over time depending on the trajectory of the pandemic as a function of the country specific active infected cases, that is, $\delta_{j}^{0 c}=\delta_{j}^{0 c}\left(I_{c, t}\right)$. The resulting sector specific pricing equation

\footnotetext{
${ }^{15}$ The $i$ th element of $I_{c, t}$ corresponds to $I_{i c, t}$. With a slight abuse of notation, we start indexing the vector with 0.
} 
takes the following form:

$$
p_{0 c, t}=\left[\sum_{j \in \mathcal{N}}\left(\delta_{j}^{0 c}\left(I_{c, t}\right)\right)^{\sigma} \Omega s_{j}^{0 c}\left(p_{j}^{0 c, t}\right)^{1-\sigma}\right]^{\frac{1}{1-\sigma}} .
$$

For each country $c$, the sector specific demand shifters are normalized to satisfy:

$$
\sum_{j \in \mathcal{N}} \delta_{j}^{0 c}\left(I_{c, t}\right) \Omega s_{j}^{0 c}=1
$$

\subsection{Solution}

We solve our model in a similar way to Baqaee and Farhi (2019), where the higher order terms are incorporated in the system via cross-factor and industry elasticities. By applying small perturbations to the system, and following the trajectory of the prices and wages, we arrive at a new equilibrium where the system re-optimizes around the old one. This methodology is akin to the Euler integration method to calculate the solutions of differential equations. Hence, we calculate differential exact hat-algebra to characterize the changes in the system by iterative means.

To perform the exact-hat-algebra, we need to trace through the changes in prices and expenditure shares after we introduce pandemic driven sectoral shocks. To feed these sectoral shocks into the model, we make use of the epidemiological model, since sectoral shocks evolve as a function of sectoral infections. Most infection dynamics models, including Acemoğlu et al. (2021), Alvarez et al. (2021), Farboodi et al. (2021), and Eichenbaum et al. (2021), do not use the sectoral heterogeneity in disease dynamics.

Our model relies on tracing the sectoral pandemic shocks to the supply term $-\Delta_{i c}\left(I_{c, t}\right)$ in Equation (13) - and demand term $-\delta_{j}^{0 c}\left(I_{c, t}\right)$ in Equation (14)- through inter-country inputoutput linkages. Let's define the Domar weight of an industry or a factor $j m$ to be: $\lambda_{j m} \equiv$ $\frac{p_{j m} y_{j m}}{E} .{ }^{16}$ Starting from Equation (7), using the expenditures for each country ( $E_{c}$ defined in Equation 4) we can write :

$$
\begin{aligned}
\lambda_{j m} & \equiv \frac{p_{j m} y_{j m}}{E}=\sum_{c \in \mathcal{C}} \frac{p_{j m} x_{j m}^{0 c}}{E_{c}} \frac{E_{c}}{E}+\sum_{k c \in \mathcal{C} \mathcal{N}} \frac{p_{j m} x_{j m}^{k c}}{E} \\
& =\sum_{c \in \mathcal{C}} \Omega_{j m}^{0 c} \chi_{c}+\sum_{k c \in \mathcal{C} \mathcal{N}} \Omega_{j m}^{k c} \frac{p_{k c} y_{k c}}{E}=\sum_{c \in \mathcal{C}} \Omega_{j m}^{0 c} \chi_{c}+\sum_{k c \in \mathcal{C} \mathcal{N}} \Omega_{j m}^{k c} \lambda_{k c},
\end{aligned}
$$

\footnotetext{
${ }^{16}$ For a factor $f, y_{f}=L_{f}$ and $p_{f}=w_{f}$
} 
where

$$
\chi_{c} \equiv E_{c} / E \equiv \sum_{f \in \mathcal{F}_{c}} \lambda_{f}
$$

denotes the country c's expenditure share in the world. Note that this equality holds for a $j m \in \mathcal{F}$ because $\Omega_{f}^{0 c}=0$.

Suppose $\Omega^{0}$ corresponds the $C \times(C N+C F)$ matrix of consumption (with last $C F$ columns all zeroes) whose entries defined in Equation (2), and $\Omega^{\mathcal{N F}}$ corresponds to the $(C N+C F) \times(C N+C F)$ matrix for the input output linkages between industries and factors whose entries given in Equations (1) and (3). Then Equation (15) can be written as:

$$
\lambda^{\prime}=\chi^{\prime} \Omega^{0}+\lambda^{\prime} \Omega^{\mathcal{N F}},
$$

where $\lambda^{\prime}$ is the $(C N+C F)$ dimensional column vector for the Domar weights of industries and $\chi$ is the $C$ dimensional column vector for country income shares. Solving this equation gives us:

$$
\lambda^{\prime}=\chi^{\prime} \Omega^{0}\left(I-\Omega^{\mathcal{N F}}\right)^{-1}=\chi^{\prime} \Omega^{0} \Psi^{\mathcal{N F}},
$$

where $\Psi^{\mathcal{N F}} \equiv\left(I-\Omega^{\mathcal{N F}}\right)^{-1}$ is the Leontief Inverse. Any small price perturbation for the system, would result in changes in the Domar weights. In particular, we can write: ${ }^{17}$

$$
\begin{aligned}
d \lambda^{\prime} & =d \chi^{\prime} \Omega^{0} \Psi^{\mathcal{N F}}+\chi^{\prime} d \Omega^{0} \Psi \mathcal{N \mathcal { F }}+\chi^{\prime} \Omega^{0} d \Psi^{\mathcal{N F}} \\
& =\left(d \chi^{\prime} \Omega^{0}+\chi^{\prime} d \Omega^{0}+\lambda^{\prime} d \Omega^{\mathcal{N F}}\right) \Psi^{\mathcal{N F}}
\end{aligned}
$$

On the cost side, with Shepard's Lemma, we can write all the price changes in terms of wage changes. Using our underlying assumptions about the production and consumption functions, we can write the changes in the $\Omega \mathrm{s}$ in terms of changes in prices and wages, which in turn could be written as function of wages of factors.

Focusing on the Domar weights of factors, we can also relate them to changes in prices

${ }^{17}$ The last equality comes from the fact that:

$$
d \Psi^{\mathcal{N F}}=d\left(I-\Omega^{\mathcal{N F}}\right)^{-1}=\Psi^{\mathcal{N} \mathcal{F}} d \Omega^{\mathcal{N F}} \Psi^{\mathcal{N F}} .
$$

To see this (dropping the superscripts):

$d\left(\Psi \Psi^{-1}\right)=0=d \Psi \Psi^{-1}+\Psi d \Psi^{-1} \Rightarrow d \Psi \Psi^{-1}=-\Psi d \Psi^{-1} \Rightarrow d \Psi=-\Psi d \Psi^{-1} \Psi=-\Psi d(I-\Omega) \Psi=\Psi d \Omega \Psi$. 
and labor supply changes with:

$$
d \lambda_{f}=\lambda_{f}\left(d \log w_{f}+d \log L_{f}\right)
$$

We can also write the changes in the country income level in terms of the changes in factor income:

$$
d \chi_{c}=\sum_{f \in \mathcal{F}_{c}} d \lambda_{f}
$$

Hence, we can write both sides of Equation (17) as a function of changes in wages and labor supply. Consequently, we can pin down the changes in wages for any factor using the following proposition:

Proposition 1 Given the supply shock $d \log L_{f}$, which is a small step towards the overall supply shock $\Delta_{f}\left(I_{c_{f}, t}\right)$, where $c_{f}$ denotes the country that factor $f$ belongs to, and the demand shock $\omega_{j}^{0 c}$, which is also a small step toward the overall demand shock $\delta_{j}^{0 c}\left(I_{c, t}\right)$, the changes in the wages can be calculated as:

$$
d \log w_{f}=\sum_{j m \in \mathcal{C} \mathcal{N}} \sum_{c \in \mathcal{C}} \chi_{c} \Omega_{j m}^{0 c} \sigma d \log \omega_{j}^{0 c} \Psi_{f}^{j m} / \lambda_{f}
$$

Line 2: $\quad+\sum_{j m \in \mathcal{C} \mathcal{N}} \sum_{c \in \mathcal{C}} \sum_{g \in \mathcal{F}_{c}} \lambda_{g}\left[d \log w_{g}+d \log L_{g}\right] \Omega_{j m}^{0 c} \Psi_{f}^{j m} / \lambda_{f}$

Line 3: $\quad+\sum_{j m \in \mathcal{C} \mathcal{N}} \sum_{g \in \mathcal{F}} \sum_{c \in \mathcal{C}} \chi_{c} \Omega_{j m}^{0 c}\left(1-\xi_{j}^{\prime}\right) \Psi_{g}^{j m} d \log w_{g} \Psi_{f}^{j m} / \lambda_{f}$

Line 4: $\quad+\sum_{j m \in \mathcal{C} \mathcal{N}} \sum_{c \in \mathcal{C}} \sum_{v \in \mathcal{C}} \sum_{g \in \mathcal{F}} \chi_{c} \Omega_{j m}^{0 c}\left(\frac{\xi_{j}^{\prime}-\sigma}{\Omega s_{j}^{0 c}}\right) \Omega_{j v}^{0 c} \Psi_{g}^{j v} d \log w_{g} \Psi_{f}^{j m} / \lambda_{f}$

Line 5: $\quad+\sum_{j m \in \mathcal{C} \mathcal{N}} \sum_{c \in \mathcal{C}} \sum_{i v \in \mathcal{C} \mathcal{N}} \sum_{g \in \mathcal{F}} \chi_{c} \Omega_{j m}^{0 c}(\sigma-1) \Omega_{i v}^{0 c} \Psi_{g}^{i v} d \log w_{g} \Psi_{f}^{j m} / \lambda_{f}$

Line 6: $\quad+\sum_{j m \in \mathcal{C} \mathcal{N}} \sum_{k c \in \mathcal{C} \mathcal{N}} \sum_{g \in \mathcal{F}} \lambda_{k c} \Omega_{j m}^{k c}\left(1-\xi_{j}\right) \Psi_{g}^{j m} d \log w_{g} \Psi_{f}^{j m} / \lambda_{f}$

Line 7: $\quad+\sum_{j m \in \mathcal{C} \mathcal{N}} \sum_{v \in \mathcal{C}} \sum_{k c \in \mathcal{C} \mathcal{N}} \sum_{g \in \mathcal{F}} \lambda_{k c} \Omega_{j m}^{k c}\left(\frac{\xi_{j}-\varepsilon}{\Omega s_{j}^{k c}}\right) \Omega_{j v}^{k c} \Psi_{g}^{j v} d \log w_{g} \Psi_{f}^{j m} / \lambda_{f}$

Line 8: $\quad+\sum_{j m \in \mathcal{C} \mathcal{N}} \sum_{k c \in \mathcal{C} \mathcal{N}} \sum_{i v \in \mathcal{C} \mathcal{N}} \sum_{g \in \mathcal{F}} \lambda_{k c} \Omega_{j m}^{k c}\left(\frac{\varepsilon-\phi}{1-\alpha_{k c}}\right) \Omega_{i v}^{k c} \Psi_{g}^{i v} d \log w_{g} \Psi_{f}^{j m} / \lambda_{f}$

Line 9: $\quad+\sum_{j m \in \mathcal{C} \mathcal{N}} \sum_{k c \in \mathcal{C} \mathcal{N}} \sum_{g \in \mathcal{F}} \lambda_{k c} \Omega_{j m}^{k c}(\phi-1) \Psi_{g}^{k c} d \log w_{g} \Psi_{f}^{j m} / \lambda_{f}$ 


$$
\text { Line 10: } \quad+(1-\phi) d \log w_{f}+(\phi-1) \sum_{g \in \mathcal{F}} \Psi_{g}^{i_{f}} d \log w_{g}-d \log L_{f} \text {. }
$$

The proof of this proposition is provided in the online Appendix and requires careful differentiation at each step, combined with the Shepard's Lemma. The intuition behind each line is as follows:

Line 1: Direct effect of the sectoral demand shock on prices, which is further translated into a factor price change via Leontief inverse.

Line 2: Consequence of changes in income shares of countries on the wages of other countries from the demand side.

Line 3: Substitution between two factors that are used in the same industry in consumption. Since our factors are sector-specific, only the term with $g=f=f_{j m}$ would survive for every $j m \in \mathcal{C N}$, where $f_{j m}$ is the factor used in industry $j m$.

Line 4: Substitution between two varieties coming from different countries in the same industry in the consumption bundles.

Line 5: Overall substitution between any two industries in consumption.

Line 6: Substitution between two factors that are used in the same industry in production. Since our factors are sector-specific, only the term with $g=f=f_{j m}$ would survive for every $j m \in \mathcal{C N}$, where $f_{j m}$ is the factor used in industry $j m$.

Line 7: Substitution between two varieties coming from different countries in the same industry in industry bundles in production.

Line 8: Substitution between two industries in intermediate bundles.

Line 9: Substitution between two industries in overall production.

Line 10: First term captures the direct effect of wage changes. The second term comes from the substitution between two factors in production. The last term captures the effect of labor loss on the wages.

Given the supply and demand shocks, unknowns in this equation are $d \log w_{f}$ terms. All can be combined to form:

$$
d \log w=A d \log w+B
$$


where $A$ and $B$ are matrices summarizing all linear relationships in Equation (18). However, the equations are not all independent from each other. To solve the system, we impose a normalization to the overall world expenditure such that:

$$
E=1 \Rightarrow d E=0=\sum_{f \in \mathcal{F}} d \lambda_{f}=\sum_{f \in \mathcal{F}} \lambda_{f}\left(d \log w_{f}+d \log L_{f}\right)
$$

Since $\lambda_{f}$ is known and $d \log L_{f}$ is an exogenous shock, this equation only scales changes in factor prices with a multiplicative factor but does not affect real output changes in sectors. As we do not compare prices between pre-Covid-19 and Covid-19 epochs, this normalization does not affect our results presented below. With the normalization, we can solve for $d \log w_{f}$ using this linear system. From the changes in log-wages, we can calculate all other log-changes in Domar weights, prices, $\Omega$ matrix, Leontief-inverse matrix and real GDP. From changes in factor prices and changes in labor supply changes, we can calculate changes in factor Domar weights. From the changes in factor Domar weights, we can calculate the changes in each country's income share, $\chi$ vector. On the other hand, using the Shepard's Lemma, we can easily find the changes good prices:

$$
d \log p_{j m}=\sum_{f \in \mathcal{F}} \Psi_{f}^{j m} d \log w_{f} .
$$

From changes in good prices and changes in factor prices, we can calculate the changes in consumption shares, input-output shares and value-added share for each industry using the underlying elasticities in our nested production and consumption functions. Once we have these changes as well, we can calculate the changes in Domar weights for goods using Equation (17). Given the price change and the Domar weight change, we can calculate the real production change in each industry as:

$$
d \log Y_{i c}=d \log \lambda_{i c}-d \log p_{i c}
$$

Similarly, we can express the real GDP change of country $c$ (defined in Equation 5) using the model primitives $\chi_{c}$ (defined in Equation 16) and $p_{0 c}$ (defined in Equation 6) as:

$$
d \log \mathrm{GDP}_{c}=d \log \chi_{c}-d \log p_{0 c}
$$

which is the total expenditure of country $c$ divided by the consumption price index of country $c, p_{0 c}$. Hence, the real GDP change that we measure at the country level is endogenously determined by the model. To compare the post-pandemic real GDPs with the pre-pandemic 
levels, we use Tönrqvist price index. This is a convenient tool to adapt for our framework because we chain small shocks to obtain a solution as in Baqaee and Farhi (2019). We provide the detailed calculations in the Section D of the Online Appendix.

\section{Data and Parameters}

\subsection{Network and Employment}

We use OECD ICIO Tables as of 2018 for 65 countries and 35 sectors, as depicted in Figure 1 in the introduction. ${ }^{18}$ These tables report expenditure shares of each sector to/from other sectors across countries. Hence they capture global input-output links or sectoral exports/imports. We obtain sectoral employment data from the OECD's Trade in employment (TiM) database (Horvàt et al., 2020). ${ }^{19}$

To calculate the industry level teleworkable share and the physical proximity measures, we use the occupational composition of the industries. We use the list provided by Dingel and Neiman (2020) for the occupations which can fulfill their tasks remotely. For the workers that continue to perform their jobs on-site, we assume that the infection rate depends on the physical proximity that is required in their workplace. To calculate the proximity requirements for the occupations, we use the self-reported Physical Proximity values available in the Work Context section of the O*NET database. O*NET collects the physical proximity information through surveys with following categories: (1) I don't work near other people (beyond $100 \mathrm{ft}$.); (2) I work with others but not closely (e.g., private office); (3) Slightly close (e.g., shared office); (4) Moderately close (at arm's length); (5) Very close (near touching). We divide the category values by 3 to make category (3) our benchmark. Specifically, a proximity value larger than 1 indicates a closer proximity than the 'shared office' level and a value smaller than 1 corresponds to less-dense working conditions. We calculate the proximity values after removing the teleworkable portion from the employees. We create a single proximity value for each occupation by weighting the normalized score with the percentage of the answers in each category.

\footnotetext{
${ }^{18}$ As the industrial classification, OECD uses an aggregation of 2-digit ISIC Rev 4 codes to 36 sectors. The last sector, "Private households with employed persons," does not have any linkages with other industries. We drop that sector from our analysis when we measure international inter-industry linkages. This leaves us with 35 sectors. Throughout our analysis, we will make use of this classification labeled as OECD ISIC Codes.

${ }^{19}$ For 14 countries that have missing data in the TiM database, we obtained the total employment data from the World Development Indicators database of the World Bank. We then gathered the value added per employer data from the closest geographical aggregation and used this information to distribute the employment to industries of these 14 countries.
} 
To obtain industry-level teleworkable share and proximity values, we calculate the weighted average of the values corresponding to the occupations in each industry using the Occupational Employment Statistics (OES) provided by the U.S. Bureau of Labor Statistics (BLS). OES data follows four-digit NAICS codes to classify industries. In order to convert proximity data to OECD ISIC codes, we make use of the correspondence table between 2018 NAICS and ISIC Revision 4 Industry Codes, provided by the U.S. Census Bureau. We show the teleworkable share and the proximity index for the industries in Table C.3 of the Appendix.

\subsection{Consumption}

OECD ICIO Tables provides us with input demand of industry $i$ in country $c$ from any industry in any country. The final demand vector has 2340 entries indexed by $i c$, corresponding to each country-industry combination. By dividing the rows of ICIO matrix with the total output of industry $i c$, we obtain the direct requirements matrix $\Omega$. This matrix summarizes the usage of each intermediate input to generate $\$ 1$ worth of output. Output of each industry is either used as an intermediate input or consumed as final output.

For the changes in the composition of consumption, that is the sector specific demand shifter, $\delta_{j}^{0 c}\left(I_{c, t}\right)$, in equation (14), we use sectoral personal consumption data for the U.S. and predict the changes as a function of infections. The data is at the monthly frequency and obtained from the Bureau of Economic Analysis (BEA) for the period from February 2020 until the end of 2020 (See Figure C.5a). We do not have the corresponding data for the other countries. Therefore, we first run a non-parametric regression for the U.S. sectoral personal consumption on the U.S. sectoral infections (a second order polynomial). Using these estimates, we calculate the sector specific consumption changes in the other countrysectors using their own sectoral infections. ${ }^{20}$

We performed two robustness checks on these estimated sectoral consumption data for the other countries. The results are shown in the Appendix. First, we report the $\mathrm{R}^{2}$ to see if more of the variation in consumption is explained by the infections in services sectors. Indeed, this turns out to be the case as shown in Figure C.5b. Second, for Turkey where we have detailed credit card spending data by sector, we compare our estimated sectoral consumption changes based on infections, with the consumption changes based on credit

\footnotetext{
${ }^{20}$ We perform several robustness checks given the sensitivity of non-parametric estimation to functional forms and extreme values in infections data, including higher-order polynomials to capture any remaining nonlinear patterns in the consumption-infection relationship.
} 
card spending data. There is a high correlation between the two as shown in Figure C.5c.

\subsection{Elasticities of Substitution: Consumption, Production and Trade}

Figure 7 shows the nested CES structure of our model both on the production side and on the consumption side, combining Figures 2 and 3. For the closed economy, we use the same elasticities as in Baqaee and Farhi (2019). Producing "Good (Varieties)" requires combining labor and intermediate goods, which are complements. We set this elasticity to $\theta=0.6$. The "intermediate bundle" captures the aggregation of alternative inputs such as steel and textiles, which are also complementary to each other. This elasticity is set to be $\varepsilon=0.2$. This value is consistent with the estimates in the literature (Atalay, 2017) who find strong complementarities at this stage.

For an open economy, the "sector bundle" captures substitution of input varieties from different countries, such as Turkish steel, Chinese steel and the U.S. steel from the perspective of the U.S.. We use industry-specific trade elasticities measured by Caliendo and Parro (2015), in which all varieties are substitutes. We also use the elasticity estimates from Boehm et al. (2019) that make the input varieties complements. Given our multiple layer CES structure, with the use of these different elasticities we can capture both substitution / complementarity between Turkish steel and Canada lumber imported by U.S. construction industry and also substitution/complementarity between Turkish and Chinese steel for the U.S. construction sector ("sector bundle" in Figure 7). Similarly on the consumption side, we can separate between consumption of Turkish cars vs German cars by the U.S. consumers ("consumption bundle" in Figure 7).

Figure 7: Model Schematic with Nested Constant Elasticity of Substitution (CES)

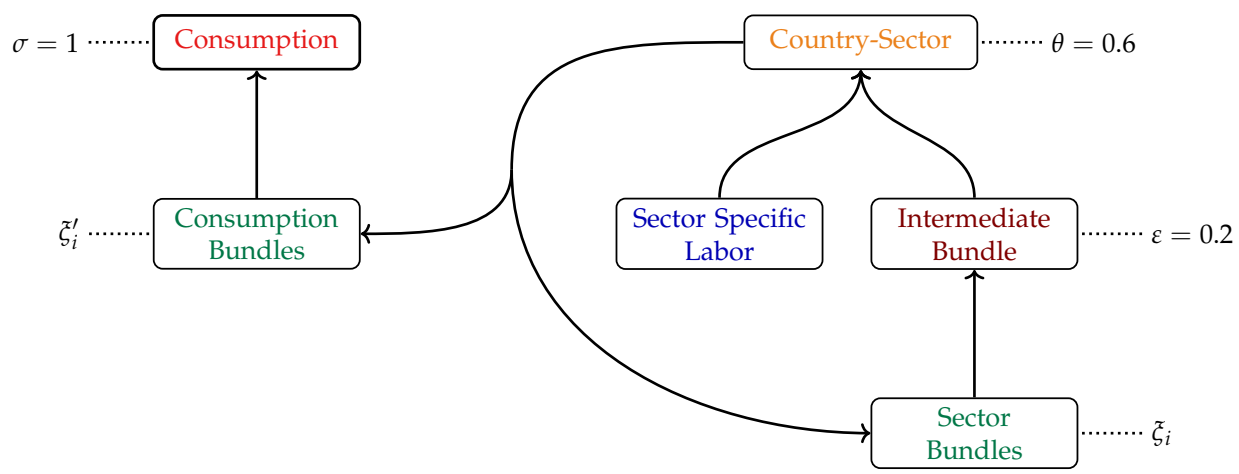




\subsection{SIR Parameters}

Each of the 65 countries in our sample have a distinct experience regarding the course of the pandemic. In the SIR model, the two fundamental structural parameters, the resolution, and the infection rates, define the pandemic's trajectory. The resolution rate is a diseasespecific structural parameter that does not vary much across the countries. According to the report by the $\mathrm{WHO}$, the median recovery time for the mild cases is approximately two weeks. ${ }^{21}$ The mean recovery time could be longer when we include severe cases. In this paper, we err on the optimistic side and set $\gamma=1 / 14 \approx 0.07$ to establish a mean recovery time of 14 days. However, the infection rate might vary across countries depending on each country's success in containing it. Furthermore, since the onset of the pandemic, the infection rate exhibits a varying pattern over time. This time variation arises because of the various lockdown measures adopted by the countries to reduce the transmission rate of the virus.

For the calibration of $\beta$, we make use of publicly available data sets to trace this variation across countries and across time. ${ }^{22}$ For each country, we estimate a SIR model described in equations (8)-(10) using official data to reproduce the variation in the trajectory of the pandemic across countries. In order to capture the variation within each country over time, we extend the SIR model to allow for time variation in the infection rate, i.e., $\beta_{t}$. Specifically, we employ the methodology proposed in Çakmaklı and Şimşek (2020) to capture the changes in the rate of infection throughout the pandemic for the countries in our sample. This methodology involves estimating a SIR model with time-varying parameters in a statistically coherent way to accommodate various non-pharmaceutical interventions, including lockdowns.

There are abundant studies that estimate the SIR model using fixed parameters (See, for example, Wu et al. (2020); Hortaçsu et al. (2021); Zhang et al. (2020)). In contrast, models that allow for time variation in parameters are scarce. Kucharski et al. (2020) uses a variant of the SIR model framework allowing the infection rate to follow a geometric random walk. Similarly, Yang et al. (2020), and Fernández-Villaverde and Jones (2022) allow for time variation in the rate of infection. The advantages of the time-varying parameters SIR model of Çakmaklı and Şimşek (2020) are twofold. First, the framework is statistically consistent with the typical count data structure related to the pandemic. This contrasts with the mod-

\footnotetext{
${ }^{21}$ https://www.who.int/docs/default-source/coronaviruse/who-china-joint-mission-on-covid-19-finalreport.pdf

${ }^{22}$ The data is downloaded from GitHub, Covid-19 Data Repository by the Center for Systems Science and Engineering (CSSE), at Johns Hopkins University.
} 
els that either employ least-squares or likelihood-based inference using normal distribution. Second, it is computationally easier, unlike the models that are statistically consistent but computationally costly, such as the particle filter. Because we exploit a wide number of countries with typically daily data, this computational efficiency is critical in our estimation process. For each country, the data spans the period from the day the number of active infections exceeds 1000 until the end of December 2020. Consequently, we use the parameter values, country-specific $\beta_{t}$, and $\gamma$ estimated as of the end of December 2020. These values reflect the stance of the pandemic for each country when the vaccines were discovered in December 2020. Using these values, we simulate the pandemic's evolution in each country over the next year. $R_{0}$ values that we use are reported in Table C.1 of the Appendix.

Our model allows for endogenous lockdown when the number of cases are over a threshold, We calibrate this threshold based on the ICU capacity of the country. The data for ICU capacities are obtained from various sources including the WHO, the JHU data repository and the health ministries of the countries. In the case of full lockdowns, the infection rate declines to zero during the lockdown, reflecting the containment of the virus. Each lockdown is imposed for 14 days. This implies that during this time, the number of Covid-19 patients decline to $36 \%$ of the value before the lockdown was imposed. Once the lockdown is removed, we assume that it takes 90 days for the infections to reach the reproduction number prior to the lockdown. Under full lockdown, only a few industries are active. We use the list of industries that are closed during lockdowns based on international examples of government decrees (see Table C.3). From these industries and using the employment data at 4 digits, we calculate the share of each OECD ISIC industry that would remain active during the lockdown. Finally, we calculate the share of public employees that are not affected by the lockdown using the publicly available information.

\section{Quantitative Results}

\subsection{Reduced Form Evidence on Supply Shocks}

Before showing results from the calibration of our model, we present two types of reduced form evidence that support the measurement of our shocks. First, we compare our infections-based sectoral supply shocks against actual data on workers who do not go to work due to pandemic related reasons. The red line in Figure 8 shows the number of these 
workers. ${ }^{23}$ Specifically, the share of the U.S. households who reported "pandemic-related" reasons for not working is 47 percent. The blue line shows our labor supply shocks for the U.S. economy. Not surprisingly, the correlation between the two series is 0.82 .

Figure 8: Labor Supply Shock

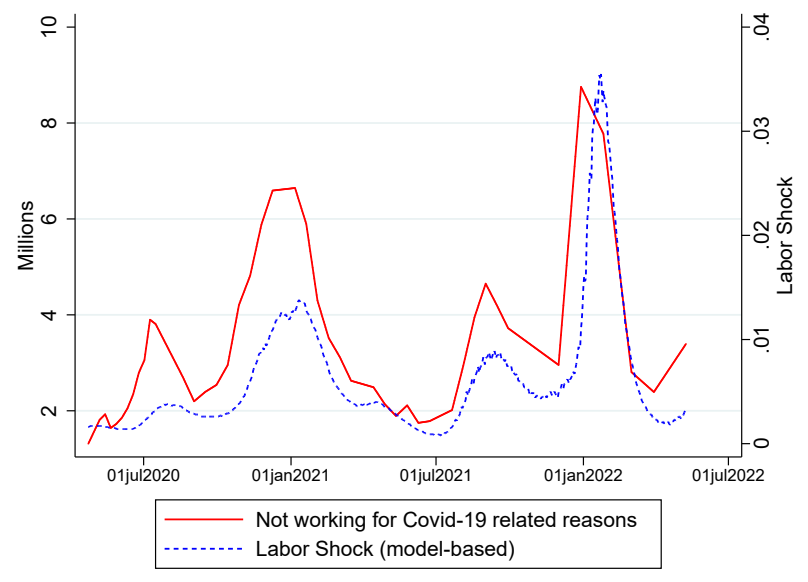

NOTES: This figure plots the co-movement of the weekly number of people who reported that they cannot work due to "pandemic-related reasons" with our labor supply shocks for the U.S. derived from the sectoral epidemiological model over the period from April 23, 2020 to May 9, 2022. See Section A.1.1 for the details on the construction of the series obtained from the U.S. Census Bureau Household Pulse Survey.

As a second robustness check, we investigate how our labor shocks co-move with supply chain disruptions observed in real-time data. For this purpose, we use the Suppliers' Delivery Time Index-a sub-index of the Purchasing Managers' Index (PMI)- and correlate it with our model-based labor shock, which is calculated by aggregating over the manufacturing industries of 26 PMI countries using trade shares as weights. We obtain a high correlation of 0.78. In Appendix Section A, we show additional evidence from other surveys on the shortage of labor and intermediate inputs for the U.S. and the Euro area countries.

\footnotetext{
${ }^{23}$ In June 2022, the Census Bureau added four questions about the current status and the duration of Covid-19 symptoms to its Household Pulse Survey, giving researchers a better understanding of the prevalence of Covid-19 symptoms. A recent Brookings Report develops a back-of-the envelope estimation to assess the impact of long Covid on the U.S. labor market, using the responses obtained from Health Table 11. According to their findings, around 16 million working-age Americans (those aged between 18 to 65) have long Covid as of August 2022. Of those, 2 to 4 million people are out of work due to long Covid. Please see https://www.brookings.edu/research/new-data-shows-long-covid-is-keeping-as-manyas-4-million-people-out-of-work/ for details of the report.
} 


\subsection{Vaccination Scenarios}

To calibrate the model we have to assume a vaccination scenario first, which determines the sectoral shocks that we feed into the model.Under full vaccination, there are no sectoral shocks. For any other scenario, there are sectoral shocks. After feeding in the sectoral shocks, we calculate the relative reduction in GDP of each country using Equation (19) as explained in detail in Section 2.6. In order to aggregate country level losses to the countrygroup-level (i.e., AEs vs EMDEs), we weight each country's output change by 2019 GDP.

We calibrate our model under two different vaccination scenarios. Table 1 summarizes these scenarios. ${ }^{24}$ The first scenario is a counterfactual scenario, where we assume that the AEs are vaccinated immediately at the onset of vaccine discovery, which corresponds to the start of our second period in the model. The EMDEs do not get vaccinated in this counterfactual scenario. The second scenario is meant to capture the real life vaccine rollout, where the AEs get vaccinated faster than the EMDEs throughout the period. The AEs complete their vaccinations sometime in the middle of the period, whereas the EMDEs, although getting vaccinated, are not able to fully inoculate their populations by the end of the period. This scenario depicts the real world rather closely as of the end of 2021.

How do we map our two period model to the rich dynamics of the second period? Our first period is pre-pandemic. We divide the second pandemic period into 365 days to mimic the heterogeneity during 2021 and solve the model daily, feeding in daily sectoral supply and demand shocks. Thanks to fast vaccination in the AEs, we stop feeding the shocks by the 120th day. As of that day, mid-2021, average number of infections decline to low levels across the AEs.

Table 1: Vaccination Scenarios

\begin{tabular}{ccc}
\hline \hline Scenario & AEs & EMDEs \\
\hline I & Immediate Complete Vaccination & No Vaccination \\
\hline II & Fast Vaccination & Slow Vaccination \\
\hline
\end{tabular}

\footnotetext{
${ }^{24}$ In both our vaccination scenarios, we assume that the vaccination is done in the same pace in different segments of the society.
} 


\subsubsection{Scenario I: Immediate Vaccination in AEs and No Vaccination in EMDEs}

With this counterfactual scenario, our goal is to illustrate the costs incurred by fully vaccinated AEs that are solely due to their trade connections with the unvaccinated world, even though they completely eliminate the pandemic at home. Thus, there are no sectoral demand and supply shocks in AEs. In EMDEs the pandemic evolves without access to any vaccine. It can only be contained by lockdowns. Countries impose lockdowns when the number of Covid-19 patients that require ICUs exceeds the number of ICUs that are reserved for Covid-19 patients.

We show the estimated real output losses for the world and by country groups in Table 2. We set both the consumption and production trade elasticities to be the same value and increase them together from row (1) to row (5) under Scenario I. Focusing on row (1), we observe that the total world real output loss can be as high as 2.2 percent of the prepandemic GDP. The first row constitutes an extreme case where the trade elasticities are set as 0.5. As the trade elasticities increase and the countries can substitute goods and inputs that are imported from different countries, overall costs decrease for everyone. The costs incurred by EMDEs are significantly higher compared to AEs because EMDEs are not vaccinated under this scenario. The share of the world output loss that is borne by the AEs go down steadily from 26 percent to 13 percent as the elasticity of substitution increases. Evidently, when there are no domestic sectoral shocks, the losses from international linkages are smaller for AEs when the elasticity of substitution is higher.

Table 2: GDP Decline Relative to the Pre-Pandemic World under Scenario I (percent)

\begin{tabular}{cccccc} 
& & \multicolumn{4}{c}{ Scenario I } \\
\hline \hline $\begin{array}{c}\text { Consumption \& Production } \\
\text { Trade Elasticities }\end{array}$ & World & AE & EMDE & $\begin{array}{c}\text { Share of } \\
\text { AEs (\%) }\end{array}$ \\
\hline (1) & $\xi_{i}^{\prime}, \xi_{i}=0.50$ & 2.149 & 0.561 & 1.588 & 26.1 \\
$(2)$ & $\xi_{i}^{\prime}, \xi_{i}=0.60$ & 1.347 & 0.312 & 1.035 & 23.2 \\
$(3)$ & $\xi_{i}^{\prime}, \xi_{i}=0.70$ & 0.996 & 0.189 & 0.806 & 19.0 \\
$(4)$ & $\xi_{i}^{\prime}, \xi_{i}=0.80$ & 0.898 & 0.140 & 0.758 & 15.6 \\
$(5)$ & $\xi_{i}^{\prime}, \xi_{i}=0.90$ & 0.857 & 0.112 & 0.744 & 13.1 \\
\hline \hline
\end{tabular}

NOTES: This table presents the real GDP losses under Scenario I. In this counterfactual scenario, we assume that the pandemic is fully contained in AEs immediately. In EMDEs the pandemic evolves at its natural course. We assume there are endogenous lockdowns in EMDEs, when the number of Covid-19 patients that require ICUs exceed the numbers of ICUs that are reserved for Covid-19 patients. Consumption and production trade elasticities, $\xi^{\prime}$ and $\xi^{\prime}$ have the same value in each row, where we increase both to capture the effect of higher substitution from row (1) to (5). 
Figure 9: Relative Decline in GDPs under Scenario I (percent)

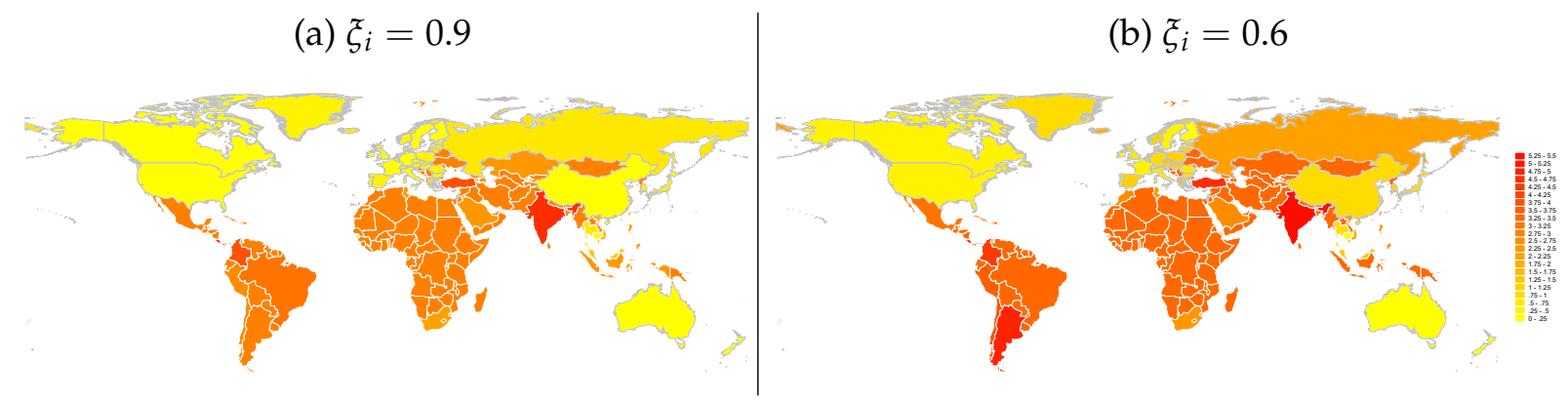

NOTES: This figure shows the reductions in relative GDP under Scenario I for two alternative trade elasticities in Panels (a)-(b), respectively. Vaccinated countries are distinguished by light gray borders.

Figure 9 displays the reductions in countries' annual real GDPs for 2021, relative to 2019, in percentage terms. Darker shades illustrate higher real output loss. Vaccinated countries are denoted by gray borders. We report two sets of estimates for comparison. High trade elasticity (the panel on the left) corresponds to values that are reported in row (5) of Table 2. Low trade elasticity (the right panel) corresponds to row (2) in the same table. As we move from a high trade elasticity to a relatively low trade elasticity, we observe that the relative reduction in real GDP increases, reflected by the darker shades. This is consistent with the intuition that lower trade elasticity reflects the difficulty in substituting among suppliers, leading to more expensive and limited imports of intermediate inputs for AEs.

\subsubsection{Scenario II: Fast Vaccination in AEs and Slow Vaccination in EMDEs}

This scenario aims to resemble the actual vaccination roll-out in real life. AEs are not fully vaccinated immediately. In addition, EMDEs have access to the vaccine, albeit at a slower rate. AEs start vaccination quite early, with the half of the susceptible population getting vaccinated in the first 30 days and the remaining half getting vaccinated in the following 90 days. Therefore, we assume that the vaccination of all susceptible population will be accomplished within 120 days in AEs. In contrast, EMDEs are not able to inoculate their susceptible populations fully, due to lack of sufficient vaccines, as was the case in 2021. Their vaccination program starts at the same time as the AEs, but it takes a full year to vaccinate half of the susceptible population. 
Table 3: GDP Decline Relative to the Pre-Pandemic World under Scenario II (percent)

Scenario II

\begin{tabular}{|c|c|c|c|c|c|}
\hline \multicolumn{2}{|c|}{$\begin{array}{c}\text { Consumption \& Production } \\
\text { Trade Elasticities }\end{array}$} & \multirow{2}{*}{$\begin{array}{c}\text { World } \\
2.687\end{array}$} & \multirow{2}{*}{$\begin{array}{c}\mathrm{AE} \\
1.038\end{array}$} & \multirow{2}{*}{$\begin{array}{c}\text { EMDE } \\
1.649\end{array}$} & \multirow{2}{*}{$\begin{array}{c}\begin{array}{c}\text { Share of } \\
\text { AEs (\%) }\end{array} \\
38.6\end{array}$} \\
\hline (1) & $\xi_{i}^{\prime}, \xi_{i}=0.50$ & & & & \\
\hline$(2)$ & $\xi_{i}^{\prime}, \xi_{i}=0.60$ (Baseline) & 1.296 & 0.592 & 0.705 & 45.6 \\
\hline (3) & $\xi_{i}^{\prime}, \xi_{i}=0.70$ & 1.125 & 0.560 & 0.565 & 49.8 \\
\hline (4) & $\xi_{i}^{\prime}, \xi_{i}=0.80$ & 1.072 & 0.550 & 0.523 & 51.2 \\
\hline (5) & $\xi_{i}^{\prime}, \xi_{i}=0.90$ & 1.049 & 0.545 & 0.504 & 51.9 \\
\hline (6) & Caliendo and Parro (2015) & 1.018 & 0.523 & 0.495 & 51.4 \\
\hline
\end{tabular}

NOTES: This table presents the real GDP losses under Scenario II. AEs follow the vaccination calendar to vaccinate the full population within four months, whereas EMDEs follow a more gradual vaccination calendar with only the half of the population getting vaccinated in one year. Consumption and production trade elasticities, $\xi^{\prime}$ and $\xi^{\prime}$ have the same value in each row, where we increase both to capture the effect of higher substitution from row (1) to (5). In the last row, we set trade elasticities to the values calculated by Caliendo and Parro (2015); these values are in general bigger than 4, implying a very high degree of substitution.

Table 3 shows the results. We observe the highest cost in row (1) with the total world real output loss of 2.6 percent of the pre-pandemic GDP. We set the two trade elasticities to 0.60 , shown in row (2), as our baseline in rest of our exercises. The baseline value of 0.6 is chosen to set the elasticities equal to the elasticity between value added and intermediate labor $(\theta)$. In our baseline case, the global output loss is close to 1.3 percent. As the trade elasticities increase, it gets easier to substitute the varieties from different countries, lowering the output losses.

When the trade elasticities exceed 0.7, the relative costs of AEs exceed more than half of the total global costs, despite the fact that they are vaccinated faster. Evidently, when both AEs and EMDEs deal with their own domestic shocks, a higher degree of substitution decreases the losses of EMDEs more than the AEs. What is the underlying intuition for this finding? According to our model, the economic costs that arise from supply chain disruptions are inversely related to: (i) The number of suppliers: The diversity of suppliers allows for alternative trade routes. Ceteris paribus, if one supplier is suffering from the pandemic, the presence of alternative trade partners allows the importer to switch to another supplier. (ii) The exposure of the suppliers to the health shock: If you have more suppliers that are vaccinated, then you are subject to less disruptions in your supply chains.

What does the data tell us? As shown in Figure 1, the EMDEs are relatively more closed economies compared to AEs. This means that EMDEs operate with fewer suppliers to begin 
with. In addition, AEs tend to trade more with the other AEs. What are the implications of these observations for the mechanics of our model summarized by (i) and (ii) above? Given that EMDEs already have less suppliers, and a larger fraction of these suppliers are unvaccinated (compared to AEs), potential disruptions in major suppliers are more likely for the EMDEs. Consequently, at low levels of elasticity of substitution, it will hit them harder because they have limited options to find alternative suppliers. The story is rather different among AEs. The exposure of AEs to a specific supplier is already less, thanks to their more diversified integration to international trade network. In a way, they do not put all their eggs in the same basket. Furthermore, because a larger fraction of their trade partners is vaccinated, they are exposed to less health shocks. Thus, at any given level of elasticity of substitution, AEs not only have a lower likelihood of health shock exposure but also they have the ability to diversify (and hence reduce) the size of the health shock. This helps them mitigate the economic costs of the pandemic.

As we increase the elasticity of substitution, EMDEs now have the flexibility to switch to another supplier when they experience major disruptions in their lifelines. This lowers their costs substantially. For the AEs, increasing the elasticity of substitution does not reduce their costs as much, because their diversified set of suppliers already reduce their reliance to a single supplier. Furthermore, because they have more vaccinated trade partners, their need to substitute (and the consequent gains from substitution) is relatively lower. Consequently, the share of AEs' costs in world GDP loss increases for higher elasticities in Scenario II, since under those parameters EMDEs' losses are lower. This is an important result highlighting the interaction between international linkages and domestic shocks and points to the fact that nationalizing supply chain may not be panacea and can even be counter-productive.

Row (6) of Table 3 shows the results for the trade elasticity values that are estimated in Caliendo and Parro (2015). These values can be perceived as long run elasticities with a higher degree of substitution. In spite of such a high degree of substitution, we obtain a similar result in terms of AEs share in world GDP losses, 50\%, compared to elasticities lower than 1 . This is due to the same compositional effect, where high degree of substitution helps EMDEs more. ${ }^{25}$

Figure 10 displays the relative reduction in countries' real GDPs under scenario II in percentage terms, again for low and high trade elasticities. The colors in both panels are

\footnotetext{
${ }^{25}$ There are endogenous lockdowns in both set of countries here. We also consider another counterfactual scenario where we do not allow for any lockdown measures. The results for this scenario are presented in Table C.5 in the Appendix.
} 
darker for AEs, reflecting higher costs. Compared to Scenario I, AEs face additional factors that work in opposite directions. On the one hand, their vaccination calendar is now slower, which increases their domestic losses. On the other hand, their EMDE trade partners now have access to the vaccine, which reduces their losses that are coming from international linkages. Comparing Figure 9 and Figure 10, AEs' slower pace of domestic vaccinations at home outweigh the trade partners' access to the vaccine, yielding a net negative impact for AEs, as we move from Scenario I to Scenario II. This is an important result that shows the right path for equitable global vaccinations: not to share limited supplies of AEs, since then domestic pandemic will stay, but rather invest in global production of vaccines.

Turning to the EMDEs, the sharp reduction in the costs borne by EMDEs is immediately noticeable with the lighter shades. This is because EMDEs also have access to the vaccine in this scenario. The darker shades for Turkey and Russia are interesting. Both of these countries are major exporters to AEs. Hence, when the AEs are subject to a slower pace of vaccinations, the economies of Turkey and Russia are also adversely affected from lower demand in AEs for their exports.

Figure 10: Relative Decline in GDPs under Scenario II (percent)

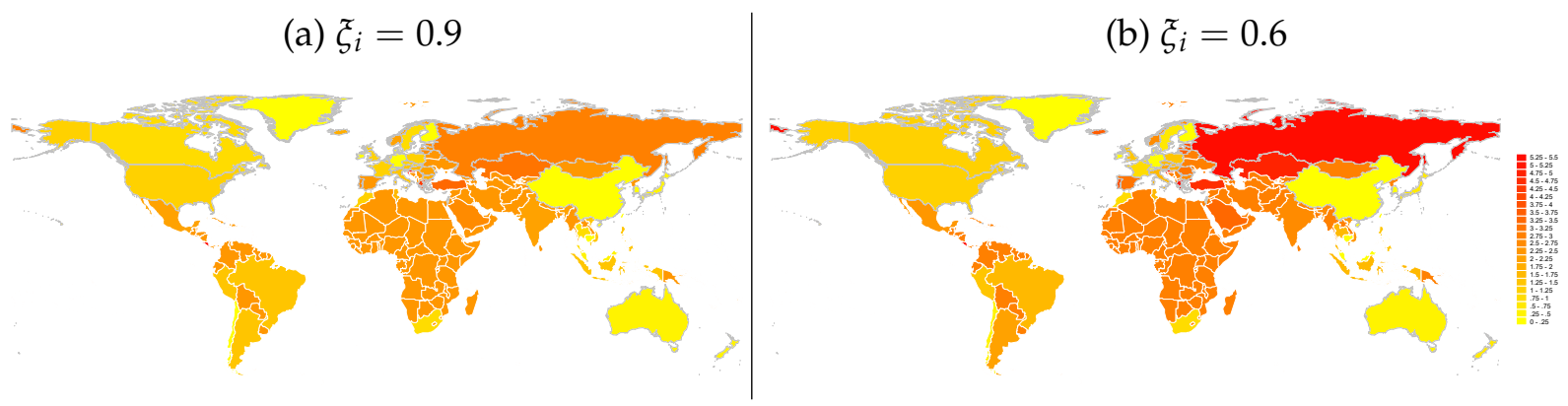

NOTES: This figure shows the reductions in relative GDP under Scenario II for two alternative trade elasticities in Panels (a)-(b), respectively. Vaccinated countries are highlighted with light gray borders.

We investigate whether the sectoral output losses are consistent with the key mechanism of the model, which should yield higher prices in supply constrained sectors. As we show in Figure C.4, the sectors where we observe larger factor price increases are the tradeable sectors, whereas services sectors see smaller price changes. To relate these model-implied changes to the actual input shortages observed in the real world, we use data available for sub-sectors of manufacturing in 21 European countries. We show that input shortages in the sector-level data of the European countries, model-implied sectoral output declines, and model-implied price increases are all significantly correlated. Table C.4 shows these sector-country regression results. Consistent with our expectations, input shortages in the 
data show a significant negative correlation with the model-implied output declines and a positive correlation with the model-implied factor price changes.

\subsection{Robustness: Counterfactual Shocks and Linkages}

We consider two counterfactuals. In the first one, we assume there is no international production network (no IPN). In the second one, we assume that there are no relative demand shocks to change the composition of consumption across sectors. The only shocks are to labor supply.

Table 4: GDP Decline Relative to the Pre-Pandemic World under Scenario II (percent): The Role of International Linkages and Demand Shocks

\begin{tabular}{lrrrc}
\multicolumn{5}{c}{ Scenario II } \\
\hline \hline & World & AE & EMDE & $\begin{array}{l}\text { Share of } \\
\text { AEs (\%) }\end{array}$ \\
\hline Baseline & 1.296 & 0.592 & 0.705 & 45.6 \\
(1) No IPN & 1.045 & 0.556 & 0.490 & 53.2 \\
(2) No DS & 0.892 & 0.398 & 0.495 & 44.5 \\
\hline \hline
\end{tabular}

NOTES: "No IPN" stands for the case where there are no International Production Networks. "No DS" stands for no sectoral demand shocks. Our Baseline scenario is with consumption and production trade elasticities of $\xi_{i}^{\prime}=0.6, \xi_{i}=0.6$, respectively. "No DS" case also uses these elasticities, whereas "No IPN" case is closed to international trade.

Table 4 displays the results for these two counterfactuals. We report the baseline findings for Scenario II for comparison purposes (top row). Row (1) shows the first counterfactual case of no international production network (no IPN). In this analysis, there are no international I-O linkages, so there is no trade for consumption and production inputs (and final goods), but only domestic I-O linkages exist. The share of AEs' costs increase to 53.2 $\%$. Why is this a higher number when we shut down the amplification role of the global network? This is because of the fact that production in AEs needs the network.

Row (2) of Table 4 shows the results from the second counterfactual of no demand shocks (no DS). We observe that in the absence of demand shocks there is a decline in real output loss both for the AEs and EMDEs, compared to the baseline. This is an interesting result. Compared to the baseline, we keep the supply shocks exactly the same and only remove the demand shocks, obtaining lower output losses. This shows that relative sectoral demand shocks amplifies the negative effects of sectoral supply shocks. 
The intuition for this result comes from the segmented labor markets. The sectors that face higher demand cannot attract more labor from other sectors, however, they do take the inputs from other sectors. Due to diminishing returns on the inputs, this results in a loss in real output. This finding is independent of complementarities and an example based on a two-sector stylized economy is provided in the Appendix Section B.

\subsection{Robustness: Asymmetric Consumption and Production Trade Elas- ticities with Domestic Complementarities}

Table 5: GDP Decline Relative to the Pre-Pandemic World (percent): The Role of Production Elasticities

\begin{tabular}{lrrrrc} 
& & \multicolumn{4}{c}{ Scenario II } \\
\hline \hline \multicolumn{2}{c}{$\begin{array}{c}\text { Consumption \& Production } \\
\text { Trade Elasticities }\end{array}$} & World & AE & EMDE & $\begin{array}{c}\text { Share of } \\
\text { AEs (\%) }\end{array}$ \\
\hline Baseline & $\xi_{i}^{\prime}=0.6, \xi_{i}=0.6$ & 1.296 & 0.592 & 0.705 & 45.6 \\
$(1)$ & $\xi_{i}^{\prime}=1.1, \xi_{i}=0.5$ & 1.607 & 0.726 & 0.881 & 45.2 \\
$(2)$ & $\xi_{i}^{\prime}=1.1, \xi_{i}=0.6$ & 1.151 & 0.585 & 0.566 & 50.8 \\
$(3)$ & $\xi_{i}^{\prime}=1.1, \xi_{i}=0.9$ & 1.039 & 0.541 & 0.498 & 52.1 \\
\hline \hline
\end{tabular}

NOTES: This table presents real output losses under the second scenario, where AEs follow a vaccination calendar to inoculate the full population within four months, whereas EMDEs follow a more gradual vaccination calendar with only the half of the population getting vaccinated. We change $\xi$, trade elasticity for the production side, but kept, $\xi^{\prime}$, the trade elasticity for the consumption side constant.

In rows (1) to (3) of Table 5 we display robustness results, where we increase the elasticity of substitution on the production side for traded inputs as before, but we leave the trade elasticity of the consumption of final goods $\xi_{i}^{\prime}$ constant, exceeding 1 . Intuitively, this exercise depicts an environment where a consumer can switch from an imported car to a domestic car under shocks. But, on the production side, if domestic car production needs foreign parts, there can be varying degrees of substitution on these parts. We obtain the same qualitative result as before that a higher degree of substitution of intermediate inputs trade helps EMDEs more than AEs and the share of AEs costs increase from rows (1) to (3), regardless of ease of substitution of final consumption goods.

Next, we undertake robustness for complementarities within the countries. Table 6, rows (1) and (2), changes the value of $\sigma$, which controls the elasticity of consumption between different domestic sectors. The baseline uses $\sigma=1$. With higher $\sigma$, output losses increase everywhere. This is due to the amplification effect of sectoral demand shocks on 
supply shocks as we have shown above under "No DS" counterfactual. When the consumers can easily substitute domestically, that relative shift intensifies the domestic supply constraints, increasing the losses. Interestingly, when $\sigma$ is lower, the losses for EMDEs increase even more. We do not have a good intuition for this result but we suspect it is due to the interaction between more severe lockdowns in EMDEs during which difficulty in consumption substitution across sectors can have more negative effects.

Table 6: GDP Decline Relative to the Pre-Pandemic World (percent): The Role of Production and Consumption Elasticities

\begin{tabular}{llcccc} 
& & \multicolumn{4}{c}{ Scenario II } \\
\hline \hline & Elasticities & World & AE & EMDE & $\begin{array}{c}\text { Share of } \\
\text { AEs (\%) }\end{array}$ \\
\hline Baseline & $\xi_{i}^{\prime}, \xi_{i}=0.60$ & 1.296 & 0.592 & 0.705 & 45.6 \\
$(1)$ & $\xi_{i}^{\prime}, \xi_{i}=0.60, \sigma=1.5$ & 1.387 & 0.658 & 0.730 & 47.4 \\
$(2)$ & $\xi_{i}^{\prime}, \xi_{i}=0.60, \sigma=0.5$ & 1.555 & 0.589 & 0.966 & 37.9 \\
$(3)$ & $\xi_{i}^{\prime}, \xi_{i}=0.60, \theta=1.5$ & 0.928 & 0.469 & 0.460 & 50.5 \\
$(4)$ & $\xi_{i}^{\prime}, \xi_{i}=0.60, \varepsilon=0.5$ & 1.131 & 0.552 & 0.580 & 48.7 \\
$(5)$ & $\xi_{i}^{\prime}, \xi_{i}=0.60, \varepsilon=1.5$ & 0.411 & 0.233 & 0.177 & 56.8 \\
$(6)$ & Cobb-Douglas & 0.818 & 0.441 & 0.377 & 53.9 \\
$(7)$ & Leontief-like & 3.448 & 1.675 & 1.773 & 48.6 \\
\hline \hline
\end{tabular}

NOTES: This table presents real output losses under the second scenario, where AEs follow the a vaccination calendar to vaccinate the full population within four months, whereas EMDEs follow a more gradual vaccination calendar with only the half of the population getting vaccinated. We change elasticities in each row. Cobb-Douglas refers to the setup where all elasticities are set to 1. Leontief refers to the setup where all elasticities are set close to 0.2 . In theory, this number could be made smaller but the non-linearity associated with such a choice makes the results unstable. Therefore, we consider this feasible lower limit as the Leontief-like setting.

In row (3), we change the value of $\theta$, which controls the substitution between labor and the intermediate bundle. In this case, when we increase the elasticity from 0.6 to 1.5 , the costs decline for everyone as the negative labor supply shock is more easily substituted by intermediate inputs. When we increase $\varepsilon$, in rows (4) and (5), which is the elasticity of substitution between sector bundles forming the intermediate bundle, from 0.2 to 0.5 (row (4)) and later to 1.5 (row (5)), the costs decrease with higher substitutability but again increasing the share of AEs in world GDP losses.

In rows (6) and (7), we show the results for two extremes, Cobb-Douglas and Leontieflike, for both consumption and production trade elasticities. ${ }^{26}$ Both AE and EMDE losses are much lower under Cobb-Douglas than Leontief-like, with a much higher degree of substitution under Cobb-Douglas. However the share of the world GDP losses borne out by

\footnotetext{
${ }^{26} \sigma=1, \theta=0.6$, and $\varepsilon=0.2$ here as in the baseline.
} 
AEs is higher under Cobb-Douglas, again as substitutability helps EMDEs more.

\section{Conclusion}

A global distribution of vaccines in the face of a global pandemic is primarily a humanitarian responsibility. Our paper makes the economic case for it. Using a structural model combined with economic and epidemiological data, we show that increasing the production of vaccines and providing it to the whole world produces significant economic benefits for the AEs who had the early access to the vaccines. Such investments provide a rate of return of 2.6 for the AEs, smoothing out the economic impact of the pandemic everywhere.

Our multi-sector-multi-country global network model is calibrated using sectoral supply and demand shocks for 65 countries and 35 sectors. These shocks are measured with predicted changes in sectoral consumption demand and labor supply as a function of sectoral infections. We measure sectoral infections using an epidemiological framework combined with country-level disease dynamics, allowing for endogenous lockdowns triggered by lack of vaccinations. Sectoral shocks travel through the global trade and production network from unvaccinated to vaccinated countries resulting in supply chain disruptions. We provide upper and lower bound estimates for the negative output effects of global supply chain disruptions, depending on the degree of complementarity across domestic and foreign factors of production and consumption of final goods.

If there are strong complementarities in the global network, even if AEs vaccinate themselves immediately, they account for a quarter of the world's real output loss. If complementarities within the network are weak, however, AEs account for only 13 percent of the world's real output loss, under the same scenario. In a realistic case where AEs are fully vaccinated by mid-year, they account for half of the world real output loss, even when factors of production and consumption goods are substitutes. Sectoral demand and supply shocks have non-linear effects, where sectoral demand shocks amplify sectoral supply shocks, regardless of elasticity of substitution. Complementarity within the global network by itself amplifies supply shocks and smooths out demand shocks.

Early in the pandemic, World Health Organization (WHO) Director Dr. Tedros Ghebreyesus and the President of the European Commission Dr. Ursula von der Leyen noted that "None of us will be safe until everyone is safe." Our findings extend this argument to the economies by showing that no economy fully recovers unless every economy recovers. We show that globalization acts both as an amplifier but also as a smoother of sectoral 
shocks. Hence, de-globalization by reducing the number of suppliers can be counterproductive. The sufferings from other people's losses is summarized by John Donne's eloquent expression that "No man is an island." Our findings in this paper reveal an economic counterpart to this expression where "No economy is an island."

\section{References}

Acemoğlu, Daron, Victor Chernozhukov, Iván Werning, and Michael D Whinston, "Optimal targeted lockdowns in a multigroup SIR model," American Economic Review: Insights, 2021, 3 (4), 487-502.

Allen, Linda JS, "A primer on stochastic epidemic models: Formulation, numerical simulation, and analysis," Infectious Disease Modelling, 2017, 2 (2), 128-142.

Alvarez, Fernando, David Argente, and Francesco Lippi, "A simple planning problem for COVID-19 lock-down, testing, and tracing," American Economic Review: Insights, 2021, 3 (3), 367-82.

Atalay, Enghin, "How important are sectoral shocks?," American Economic Journal: Macroeconomics, 2017, 9 (4), 254-80.

Atkeson, Andrew, "How Deadly is COVID-19? Understanding the Difficulties with Estimation of its Fatality Rate," Working Paper 26965, National Bureau of Economic Research 2020.

Baqaee, David and Emmanuel Farhi, "Networks, barriers, and trade," Working Paper 26108, National Bureau of Economic Research 2019.

_ and _ , "Supply and demand in disaggregated keynesian economies with an application to the COVID-19-19 crisis," American Economic Review, 2022, 112 (5), 1397-1436.

Bendavid, Eran and Jay Bhattacharya, "Is Coronavirus as Deadly as They Say?," Wall Street Journal, 2020, 24.

Boehm, Christoph E, Aaron Flaaen, and Nitya Pandalai-Nayar, "Input linkages and the transmission of shocks: Firm-level evidence from the 2011 Tōhoku earthquake," Review of Economics and Statistics, 2019, 101 (1), 60-75.

_, Andrei A Levchenko, and Nitya Pandalai-Nayar, "The long and short (run) of trade elasticities," American Economic Review, forthcoming.

Çakmaklı, Cem and Yasin Şimşek, "Bridging the COVID-19 Data and the Epidemiological Model using Time Varying Parameter SIRD Model," Working Paper series 20-23, Rimini Centre for Economic Analysis July 2020. 
Caliendo, Lorenzo and Fernando Parro, "Estimates of the Trade and Welfare Effects of NAFTA," The Review of Economic Studies, 2015, 82 (1), 1-44.

Chetty, Raj, John N Friedman, Nathaniel Hendren, Michael Stepner, and The Opportunity Insights Team, "How Did COVID-19 and Stabilization Policies Affect Spending and Employment? A New Real-Time Economic Tracker Based on Private Sector Data," Working Paper 27431, National Bureau of Economic Research 2020.

Costinot, Arnaud and Andrés Rodríguez-Clare, "Trade theory with numbers: Quantifying the consequences of globalization," in "Handbook of International Economics," Vol. 4, Elsevier, 2014, pp. 197-261.

Dewatripont, Mathias, Michel Goldman, Eric Muraille, and Jean-Philippe Platteau, "Rapidly identifying workers who are immune to COVID-19 and virus-free is a priority for restarting the economy," Working Paper, VOX CEPR Policy Portal 2020.

di Giovanni, Julian, Şebnem Kalemli-Özcan, Alvaro Silva, and Muhammed Ali Yıldırım, "Global Supply Chain Pressures, International Trade, and Inflation," Working Paper 30240, National Bureau of Economic Research 2022.

Dingel, Jonathan I and Brent Neiman, "How many jobs can be done at home?," Journal of Public Economics, 2020, 189, 104235.

Eichenbaum, Martin S, Sergio Rebelo, and Mathias Trabandt, "The macroeconomics of epidemics," The Review of Financial Studies, 2021, 34 (11), 5149-5187.

Farboodi, Maryam, Gregor Jarosch, and Robert Shimer, "Internal and external effects of social distancing in a pandemic," Journal of Economic Theory, 2021, 196, 105293.

Fauci, AS, HC Lane, and RR Redfield, "COVID-19-Navigating the Uncharted.," The New England Journal of Medicine, 2020, 382 (13), 1268-1269.

Fernald, John and Huiyu Li, "The Impact of COVID on Productivity and Potential Output," 2022. Jackson Hole Economic Symposium.

Fernández-Villaverde, Jesús and Charles I Jones, "Estimating and simulating a SIRD model of COVID-19 for many countries, states, and cities," Journal of Economic Dynamics and Control, 2022, p. 104318.

Goolsbee, Austan and Chad Syverson, "Fear, lockdown, and diversion: Comparing drivers of pandemic economic decline 2020," Journal of Public Economics, 2021, 193, 104311.

Guerrieri, Veronica, Guido Lorenzoni, Ludwig Straub, and Iván Werning, "Macroeconomic Implications of COVID-19: Can Negative Supply Shocks Cause Demand Shortages?," American Economic Review, 2022, 112 (5), 1437-74.

Hortaçsu, Ali, Jiarui Liu, and Timothy Schwieg, "Estimating the fraction of unreported infections in epidemics with a known epicenter: An application to COVID-19," Journal of Econometrics, 2021, 220 (1), 106-129. 
Horvàt, Peter, Colin Webb, and Norihiko Yamano, "Measuring employment in global value chains," 2020. https://www.oecd-ilibrary.org/content/paper/00f7d7db-en.

IMF, “World Economic Outlook, October 2020: A Long and Difficult Ascent," 2020. International Monetary Fund, https://www.imf.org/en/Publications/WEO/Issues/2020/09/ 30/world-economic-outlook-october-2020.

Krugman, Paul R, Maurice Obstfeld, and Marc Melitz, International Economics: Theory and Policy, Pearson, 2022.

Kucharski, Adam J, Timothy W Russell, Charlie Diamond, Yang Liu, John Edmunds, Sebastian Funk, Rosalind M Eggo, Fiona Sun, Mark Jit, James D Munday, Nicholas Davies, Amy Gimma, Kevin van Zandvoort, Hamish Gibbs, Joel Hellewell, Christopher I Jarvis, Sam Clifford, Billy J Quilty, Nikos I Bosse, Sam Abbott, Petra Klepac, and Stefan Flasche, "Early dynamics of transmission and control of COVID-19: a mathematical modelling study," The Lancet Infectious Diseases, 2020, 20 (5), 553 - 558.

Li, Ruiyun, Sen Pei, Bin Chen, Yimeng Song, Tao Zhang, Wan Yang, and Jeffrey Shaman, "Substantial undocumented infection facilitates the rapid dissemination of novel coronavirus (SARS-CoV2)," Science, 2020.

OECD, “OECD Inter-Country Input-Output (ICIO) Tables,” 2020. https:/ /www.oecd.org/ sti/ind/inter-country-input-output-tables.htm.

Vogel, Gretchen, "New blood tests for antibodies could show true scale of coronavirus pandemic," Science, March 192020.

Wu, Joseph T, Kathy Leung, and Gabriel M Leung, "Nowcasting and forecasting the potential domestic and international spread of the 2019-nCoV outbreak originating in Wuhan, China: a modelling study," The Lancet, 2020, 395 (10225), 689-697.

Yang, Qihui, Chunlin Yi, Aram Vajdi, Lee W Cohnstaedt, Hongyu Wu, Xiaolong Guo, and Caterina M Scoglio, "Short-term forecasts and long-term mitigation evaluations for the COVID-19 epidemic in Hubei Province, China," Infectious Disease Modelling, 2020, 5, 563-574.

Zhang, Yuan, Chong You, Zhenhao Cai, Jiarui Sun, Wenjie Hu, and Xiao-Hua Zhou, "Prediction of the COVID-19 outbreak in China based on a new stochastic dynamic model," Scientific reports, 2020, 10 (1), 1-10. 


\section{APPENDIX}

\section{A Reduced Form Evidence}

The EU Commission's Business and Consumer Survey provides time series data from the business managers on the factors limiting production. ${ }^{27}$ Panel A of Figure A.1 plot these factors as a weighted average of all EU countries for manufacturing, construction, and services sectors. Historically, especially during the 2008-2009 crisis, we observe that insufficient demand (blue line) is the most important factor limiting production. During times of low demand, labor and/or material shortages turn out to be nonbinding constraints. This is consistent with the intuition that when the economy is demand constrained, there is a limited role for supply chain bottlenecks. What is unique about the Covid-19 shock is that shortage of material and equipment (the green line), and labor (red line) which both became the key reasons for limiting production in all sectors, including services.

Figure A.1: Factors Limiting Production in Europe

(a1) Manufacturing

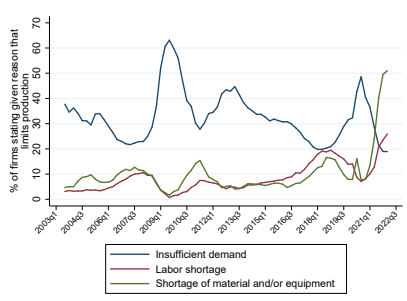

(b1) Manufacturing

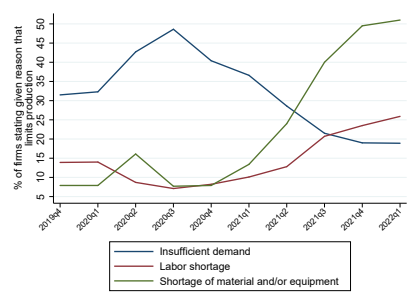

Panel A: 2003q3-2022q1

(a2) Construction

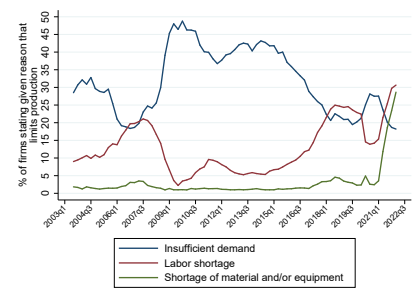

Panel B: 2019q4-2022q1

(b2) Construction

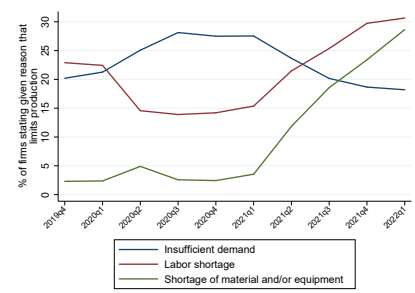

(a3) Services

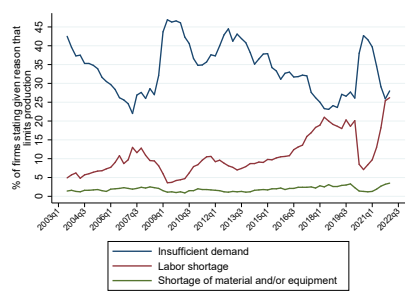

(b3) Services

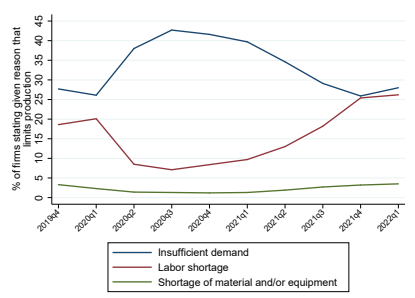

NOTES: Figure A.1 plots the replies of business managers to the question "What main factors are currently limiting your production?" as a weighted average using country shares in EU's total gross-value added. These series are smoothed by calculating a two-year moving average and they are seasonally adjusted.

\footnotetext{
${ }^{27}$ See https://ec.europa.eu/info/business-economy-euro/indicators-statistics/economic-databases / business-and-consumer-surveys_en for more information about business and consumer surveys.
} 
Figure A.1 also plots the period after $2019 \mathrm{q} 4$ to zoom-in on the asymmetric dynamics across sectors. As can be seen from Panel B of this figure, the recovery of demand, thanks to vaccinations and economic stimulus packages, worsened the labor and material shortages throughout 2021. This is consistent with the framework depicted in Guerrieri et al. (2022), who note the asymmetric nature of the pandemic and the associated spillovers among sectors. Manufacturing, a tradeable sector, registered higher shortages of inputs due to the overlap of supply chain bottlenecks and recovery in demand. The adverse effects of the pandemic were rather noticeable in this sector because most of its inputs are imported. In comparison, non-tradeable sectors such as construction and services suffer relatively more from labor shortages. Given the complementarity of intermediate inputs and labor in our framework, our estimates can capture this notion of switching from a primarily demandconstrained world to a mostly supply-constrained world during the pandemic's course.

Figure A.2: Factors Limiting Production during the Pandemic in U.S. (August 2020-May 2022)

(a1) Manufacturing

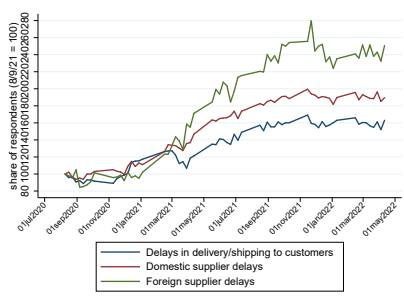

(b1) Manufacturing

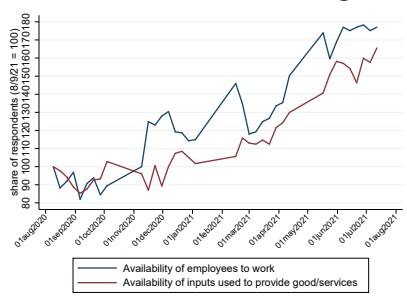

Panel A: Supplier Delays

(a2) Construction

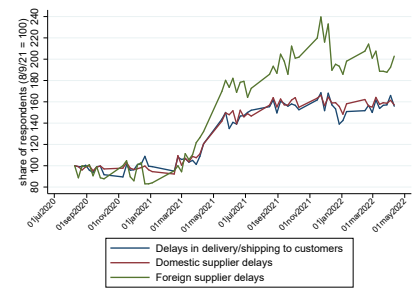

Panel B: Material and Labor Shortage

(b2) Construction

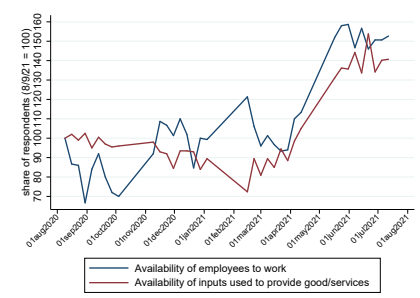

(a3) Services

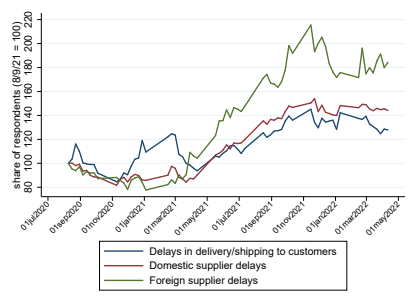

(b3) Services

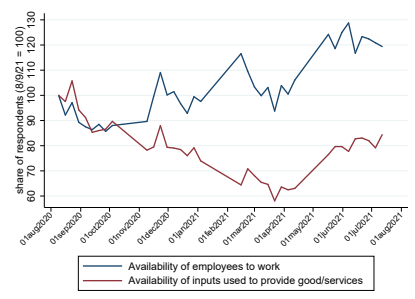

Notes: Figure A.2 plots the replies of small business managers in the U.S. Pulse Survey to the question "In the last week, was this business affected by any of the following?" This figure provides a cross-sectoral comparison of the factors that limit U.S. production during the Covid-19 era.

An analogous survey for the U.S. illustrates the factors limiting production as shown in Figure A.2. This figure highlights the importance of global supply chain bottlenecks even for the non-tradeable sectors of construction and services in panels (a2) \& (b2) and (a3) \& 
(b3), with the most important delays coming from foreign suppliers.

\section{A.1 Data Details}

\section{A.1.1 Household Pulse Survey}

The U.S. Census Bureau designed the Household Pulse Survey (HPS) with the aim of understanding the individuals' experiences of Covid-19 as well as providing timely information essential in terms of employment status, food security, housing, physical and mental health, access to health care and educational disruption. ${ }^{28}$

For the purpose of our analysis, we focus on the national level information in terms of employment status that is available in the Employment Section. ${ }^{29}$ This section asks the U.S. households the reasons for not working and counts the responses for each reason reported. Using the counts available, we first linearly interpolate the weekly/bi-weekly series into daily series. We then group the reasons for not working into the following three categories, listed in the below.

(i) Individual pandemic related reasons $(47.1 \%)$

- I was caring for someone or sick myself with coronavirus symptoms

- I did not work because I am/was caring for children not in school or daycare

- I was concerned about getting or spreading the coronavirus

- I did not work because I am/was caring for an elderly person

- I am/was sick (not coronavirus related) or disabled

(ii) Business related reasons (15\%)

- I was laid off or furloughed due to coronavirus pandemic

- My employment went out of business due to the coronavirus pandemic

\footnotetext{
${ }^{28}$ As of May 2022, the HPS had three phases: In Phase 1 U.S. households were surveyed on a weekly basis over the time period from April 23, 2020 to July 21, 2020; In Phase 2 U.S. households were surveyed on a bi-weekly basis over the time period from August 19, 2020 to October 26, 2020; In Phase 3, U.S. households were surveyed on a bi-weekly basis over the time period from October 28, 2020 to May 9, 2022.

${ }^{29}$ The HPS uses a national representative sample to produce estimates at three geographic levels: (1) 15 largest Metropolitan Statistical Areas, (2) each of the 50 states plus the District of Columbia, and (3) the national level. Each round of HPS provides "Table 3. Educational Attainment for Adults Not Working at Time of Survey, by Main Reason for Not Working and Source Used to Meet Spending Needs."
} 
(iii) Government mandated reasons (4.7\%)

- My employment closed temporarily due to the coronavirus pandemic

For reasons for not being employed, $47.1 \%$ of the U.S. population aged 18 years and older reported individual pandemic related reasons, 15\% reported business related reasons and $4.7 \%$ reported government mandated reasons. ${ }^{30}$

\section{A.1.2 Current Population Survey}

U.S. Bureau of Labor Statistics conducts the monthly household labor force survey, Current Population Survey (CPS), to track the number of employed people who missed work during the survey reference week. Specifically, this survey provides the following measures: i) people who did not work at all in the survey reference week, ii) people who usually work full time but were at work part time (1 to 34 hours) during the reference week.

Figure A.3: Reasons for Not Working, 2012m1-2022m7

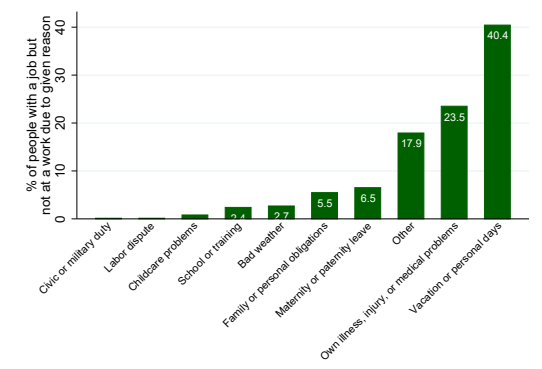

(a) averages over the sample period 2012m1-2022m7

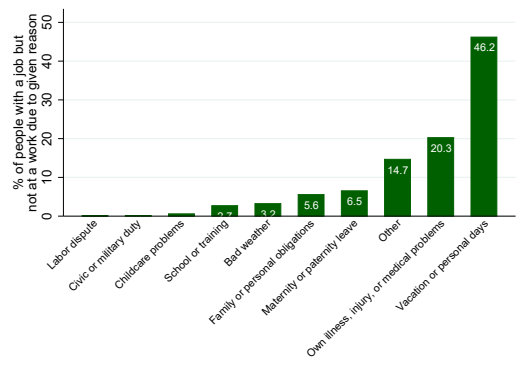

(b) pre-Covid-19 averages

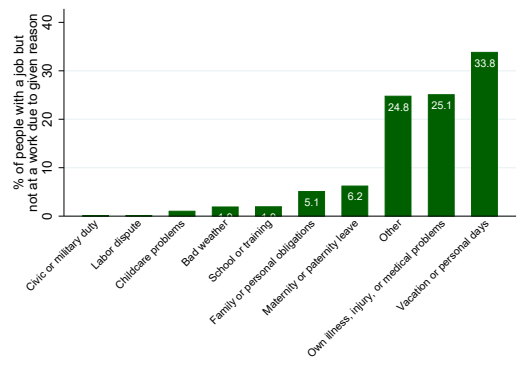

(c) post-Covid-19 averages

NOTES: Figure A.3 displays the fraction of "employed" households in each of the given categories, based on their expressed reasons for not being at work. The numbers may not be added up to 100 because of rounding in the computation of the corresponding averages.

Different from the HPS explained above, this survey provides time series data that goes until 2012. This feature enables us to compare post-Covid-19 employment figures with preCovid-19 ones. For the purpose of our analysis, we utilize the time series data on employed

\footnotetext{
${ }^{30}$ From the total U.S. population, we excluded the households who stated the reason for not working as the retirement at the time of survey. We calculate the shares by collapsing daily values into yearly values and taking the average of the resulting yearly responses over the period from 2020 to 2022. The numbers do not add up to 100 because the households stating the reason for not working as "Other" and "No response" are not considered in the computation.
} 
people with a job not at work by selected reasons. Figure A.3 displays the fraction of "employed" households in each of the given categories, based on their expressed reasons for not being at work.

\section{B Sectoral Shocks with Sector Specific Labors}

Figure B.4: Factor Levels during Pandemic

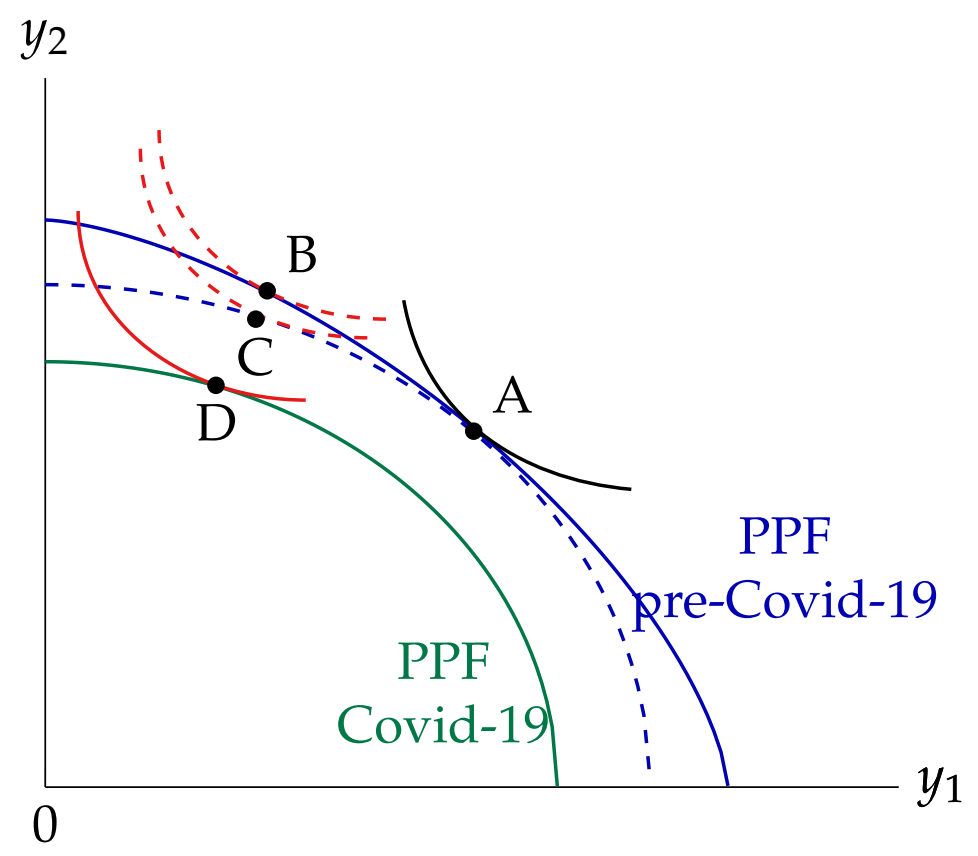

In this section, we provide an intuitive perspective to illustrate our results using a stylized two-sector economy. Figure B.4 shows the Production Possibility Frontiers (PPF) and the indifference curves of the economy under different scenarios. Before Covid-19, PPF is denoted by the blue line and the utility maximization by the indifference curve denoted by black curve, yielding an equilibrium at point A. For this PPF, we assume that the labor is mobile between the sectors. Once we make the labor supply sector specific, we arrive at the PPF represented by the dashed blue line. The dashed blue PPF lies below the pre-Covid (solid blue) PPF. This is because the dashed line corresponds to the case with an additional constraint of immobile factor (see, e.g., the specific factors model in Chapter 4 of Krugman et al., 2022). Up to this stage, the equilibrium remains intact at pre-Covid equilibrium, point A. After we introduce a sectoral demand shock, however, the utility function changes such 
that we would be optimizing along the red indifference curves. If the labor was not sector specific, the equilibrium would have moved to point $\mathrm{B}$ following the demand shock. Instead, the new equilibrium moves to point C. Furthermore, since there is also a labor supply shock in both sectors, the new PPF shifts inwards to the one represented by the green line and the equilibrium moves to point $\mathrm{D}$. With standard assumptions on indifference curves, one can deduce that the output in point $D$ is lower than point $C$, which is lower than point $B$. In standard models, pure demand shocks as moving from point $A$ to point $B$ will not deliver real output effects. But since point $C$ and point $D$ are worse off compared to point $B$, point $C$ is worse off than point $B$. To quantitatively compare the real output levels between points $A$ and $C$, we use the Törnqvist index.

\section{Additional Figures and Tables}

\section{List of Figures and Tables:}

- Figure C.1: The Structure of OECD Inter-Country Input-Output (ICIO) Table

- Figure C.2: Combined Input-Output Matrix

- Figure C.3: Proximity Index and Teleworkable Share by Industry

- Figure C.4: Distribution of Factor Price Changes

- Figure C.5: Demand Changes Across Industries

- Table C.1: Country Settings for Various Scenarios

- Table C.2: ICU Bed Capacities

- Table C.3: List of Essential Sectors during Lockdowns

- Table C.4: Relationship with Input Shortage

- Table C.5: GDP Decline Relative to the Pre-Pandemic World (percent): No Endogenous Lockdowns 
Figure C.1: The Structure of OECD Inter-Country InPut-Output Table

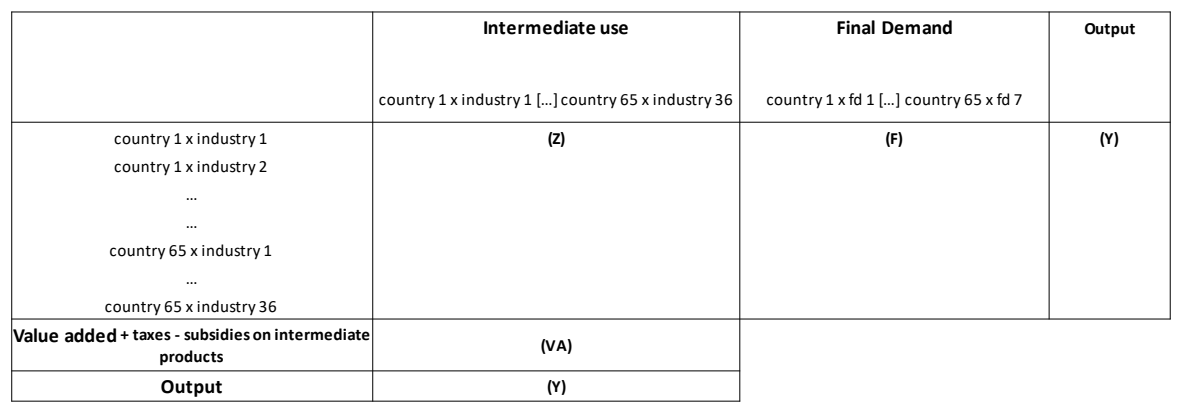

NotEs: This figure illustrates the structure of OECD Inter-Country Input-Output Table (ICIO), which represents the breakdown of output corresponding to 36 industries and 65 countries, giving us a matrix of $2340 \times 2340$ entries. In any industry-country combination, the output $(Y)$ equals intermediate use $(Z)$ plus final demand $(\mathrm{F})$ of 36 industries in 65 countries. Industry list can be found in Figure C.3. Further, in any industry-country combination, final demand sums the following components of expenditures over 65 countries. fd1: Households Final Consumption Expenditure (HFCE); fd2: Non-Profit Institutions Serving Households (NPISH); fd3: General Government Final Consumption (GGFC); fd4: Gross Fixed Capital Formation (GFCF); fd5: Change in Inventories and Valuables (INVNT); fd6: Direct purchases by non-residents (NONRES); fd7: Statistical Discrepancy (DISC).

Figure C.2: COMBINED INPUT-OUTPUT MATRIX

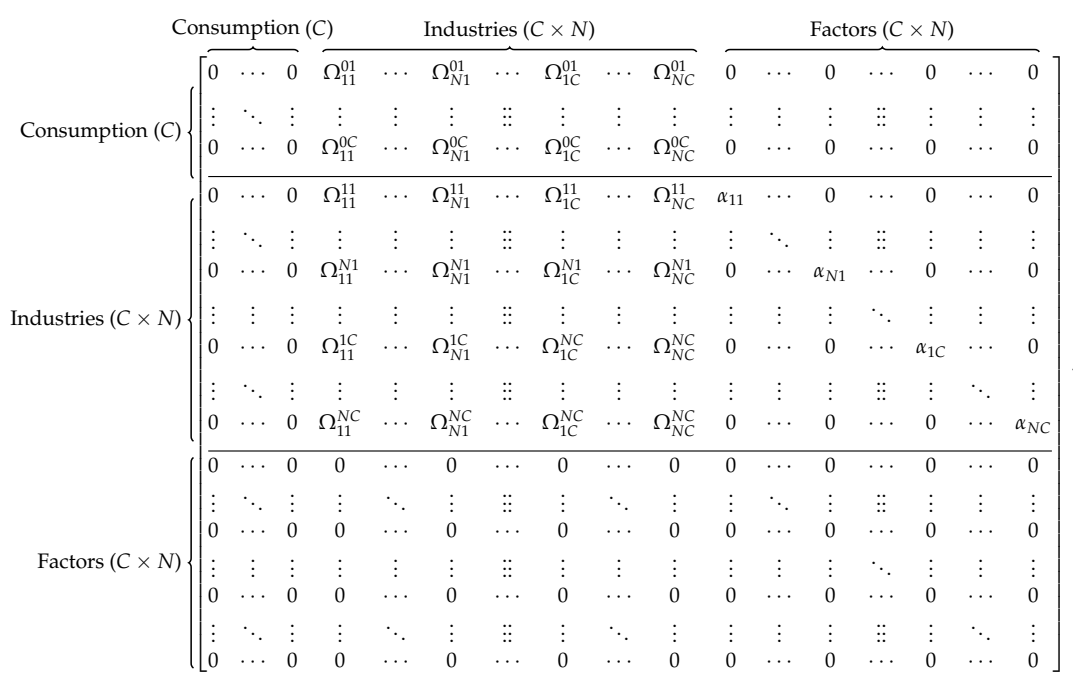

Notes: The structure of Combined Input-Output table $(\Omega)$ that we use. It includes the consumption, production and factors in a single matrix. 
Figure C.3: Proximity Index and Teleworkable Share by Industry

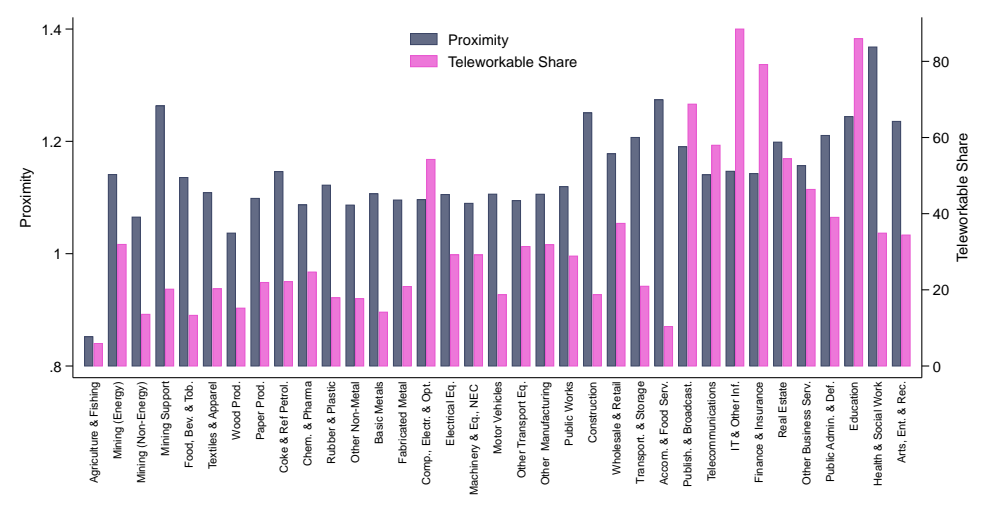

NOTES: In this figure, we present the physical proximity index, the share of teleworkable employees as well as demand changes in a given industry, which is categorized based on OECD ISIC Codes. In comparing proximity values across differential sectors listed in the first column, we use weighted average of occupation-specific proximity values in those sectors. Specifically, an occupation of a given industry is assigned with a proximity value that is smaller than 1 if it has sparse working conditions. An occupation of a given industry is assigned with a proximity value that is larger than 1 if it requires closer proximity than the "shared office" level. We calculate the proximity values for a given industry after removing the teleworkable share of the employees of that industry. Doing so, we follow Dingel and Neiman (2020)'s list of teleworkable occupations to determine the share of employees that can work remotely in each industry.

\section{Figure C.4: Factor Price Changes}

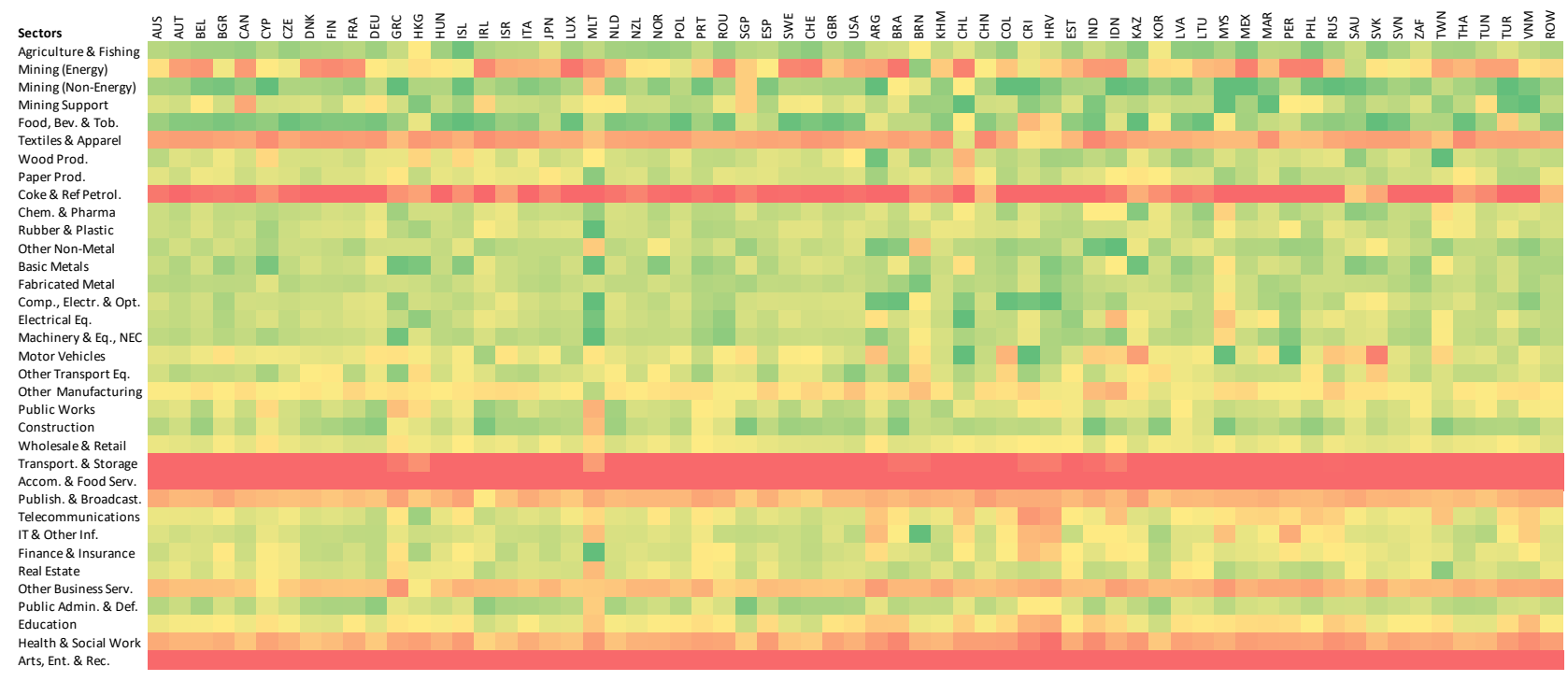

NOTES: This figure represents the distribution of factor price changes across countries for our baseline model. The sectors are shown on the rows while the countries are shown on the columns. We normalize the factor prices by subtracting the mean and dividing by the standard deviation of the changes within a country. The mean values are similar to the ones shown in Figure 10b in the main text. The color scheme is from green (starting from -1.5), to yellow (0) and to red (1.5). 
Figure C.5: Demand Shocks

(a) Sectoral Demand in the U.S.

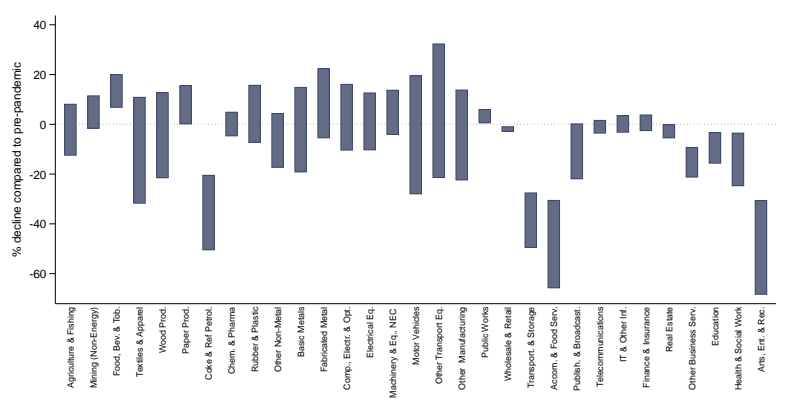

(b) Goodness-of-Fit

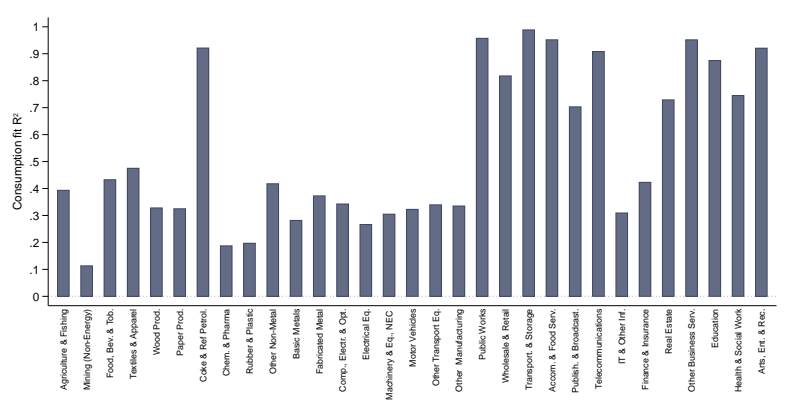

(c) Sectoral Demand-Credit Card Data

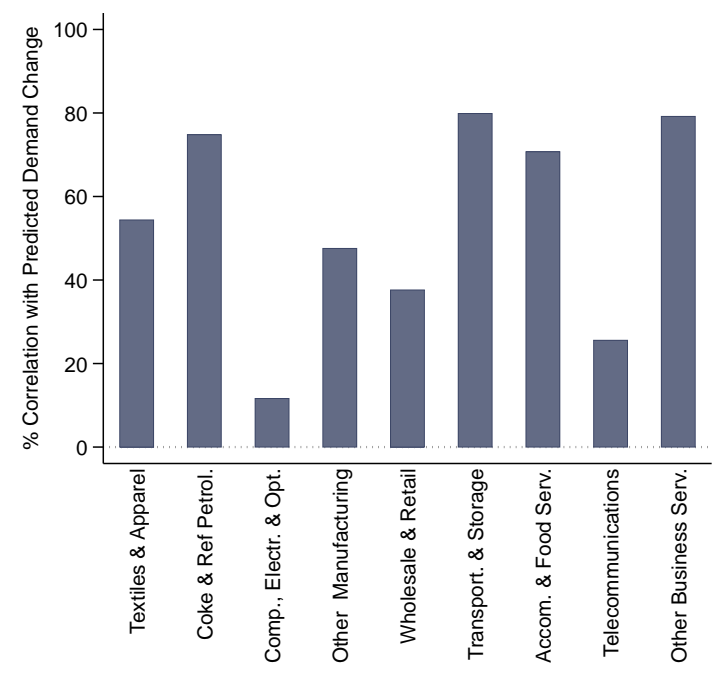

NOTES: Panel (a) shows the range of changes in sectoral personal consumption data for the U.S. (minimum and maximum observed values). The data is at monthly frequency and obtained from the Bureau of Economic Analysis (BEA) for the period from March 2020 until the end of 2021. Following Baqaee and Farhi (2022), we calculate the monthly changes in consumption relative to February 2020. To calculate demand changes, we first run regressions to fit declines in demands with a second degree polynomial of the infections. Panel (b) shows the goodness of the fit for each sector through the $\mathrm{R}^{2}$ values. There is no variation in the reported data for Mining and extraction of energy producing products, Mining support service activities, Construction and Public administration and defence; compulsory social security sectors. For expositional convenience, we do not show these industries. Panel (c) shows correlations of predicted demand changes with real-time demand changes by sector for the period from 2020w11 to $2020 \mathrm{w} 26$ (corresponding to the first three months of pandemic since the first Covid-19 case was announced in Turkey). For Turkey, we estimate predicted demand changes following the computation method explained in Section 3.2. We calculate weekly changes in credit card spending relative to four-week average value corresponding to the period from 2020w7 to 2020w10. Following the mapping between CBRT industry codes and OECD ISIC industries, we exclude the sectors for which credit card is not the common means of payment, hence the coverage is limited. 
Table C.1: COUnTRY SETTINGS FOR VARIOUS ScENARIOS

\begin{tabular}{|c|c|c|c|c|c|c|}
\hline Country & $\begin{array}{c}\text { ICU capacity } \\
\text { for } \\
\text { Covid-19 patients }\end{array}$ & $\begin{array}{l}\text { Reproduction } \\
\text { rate } \mathrm{R}_{0}\end{array}$ & $\begin{array}{c}\text { GDP } 2019 \\
\text { (Billion USD) }\end{array}$ & $\begin{array}{c}\text { Share of } \\
\text { vaccinated } \\
\text { population }\end{array}$ & $\begin{array}{l}\text { Duration of } \\
\text { vaccination } \\
\text { (days) }\end{array}$ & $\begin{array}{l}\text { Openness } \\
\text { Index }\end{array}$ \\
\hline Australia & 1665 & 0.7 & 1,393 & $100 \%$ & $120(30-90)$ & 35 \\
\hline Austria & 1000 & 1.1 & 446 & $100 \%$ & $120(30-90)$ & 81 \\
\hline Belgium & 2756 & 1.1 & 530 & $100 \%$ & $120(30-90)$ & 164 \\
\hline Canada & 2713 & 1.3 & 1,736 & $100 \%$ & $120(30-90)$ & 52 \\
\hline Chile & 1383 & 1.3 & 282 & $50 \%$ & 330 & 49 \\
\hline Czechia & 4151 & 1.1 & 247 & $100 \%$ & $120(30-90)$ & 153 \\
\hline Denmark & 925 & 1.2 & 348 & $100 \%$ & $120(30-90)$ & 60 \\
\hline Estonia & 338 & 1.2 & 31 & $100 \%$ & $120(30-90)$ & 109 \\
\hline Finland & 220 & 1.1 & 269 & $100 \%$ & $120(30-90)$ & 55 \\
\hline France & 8000 & 1.1 & 2,716 & $100 \%$ & $120(30-90)$ & 45 \\
\hline Germany & 28000 & 1.1 & 3,846 & $100 \%$ & $120(30-90)$ & 71 \\
\hline Greece & 704 & 1.1 & 210 & $100 \%$ & $120(30-90)$ & 48 \\
\hline Hungary & 1094 & 1.1 & 161 & $100 \%$ & $120(30-90)$ & 151 \\
\hline Iceland & 163 & 1.1 & 24 & $100 \%$ & $120(30-90)$ & 49 \\
\hline Ireland & 248 & 1.1 & 389 & $100 \%$ & $120(30-90)$ & 69 \\
\hline Israel & 4900 & 1.3 & 395 & $100 \%$ & $120(30-90)$ & 34 \\
\hline Italy & 7700 & 1.1 & 2,001 & $100 \%$ & $120(30-90)$ & 50 \\
\hline Japan & 3996 & 1.3 & 5,082 & $100 \%$ & $120(30-90)$ & 28 \\
\hline Korea & 5481 & 1.3 & 1,642 & $100 \%$ & $120(30-90)$ & 64 \\
\hline Latvia & 186 & 1.1 & 34 & $100 \%$ & $120(30-90)$ & 102 \\
\hline Lithuania & 451 & 1.1 & 54 & $100 \%$ & $120(30-90)$ & 127 \\
\hline Luxembourg & 91 & 1.1 & 71 & $100 \%$ & $120(30-90)$ & 57 \\
\hline Mexico & 4211 & 1.1 & 1,258 & $50 \%$ & 330 & 74 \\
\hline Netherlands & 1161 & 1.1 & 909 & $100 \%$ & $120(30-90)$ & 148 \\
\hline New Zealand & 585 & 0.7 & 207 & $100 \%$ & $120(30-90)$ & 40 \\
\hline Norway & 455 & 1.1 & 403 & $100 \%$ & $120(30-90)$ & 47 \\
\hline Poland & 3074 & 1.1 & 592 & $100 \%$ & $120(30-90)$ & 89 \\
\hline Portugal & 455 & 1.1 & 238 & $100 \%$ & $120(30-90)$ & 66 \\
\hline Slovakia & 570 & 1.1 & 105 & $100 \%$ & $120(30-90)$ & 170 \\
\hline Slovenia & 377 & 1.1 & 54 & $100 \%$ & $120(30-90)$ & 166 \\
\hline Spain & 4566 & 1.1 & 1,394 & $100 \%$ & $120(30-90)$ & 51 \\
\hline Sweden & 365 & 1.1 & 531 & $100 \%$ & $120(30-90)$ & 60 \\
\hline Switzerland & 1012 & 1.1 & 703 & $100 \%$ & $120(30-90)$ & 84 \\
\hline Turkey & 16850 & 1.3 & 754 & $50 \%$ & 330 & 52 \\
\hline United Kingdom & 7018 & 1.1 & 2,827 & $100 \%$ & $120(30-90)$ & 41 \\
\hline US & 84676 & 1.1 & 21,370 & $100 \%$ & $120(30-90)$ & 20 \\
\hline Argentina & 8404 & 1.1 & 450 & $50 \%$ & 330 & 25 \\
\hline Brazil & 43466 & 1.1 & 1,840 & $50 \%$ & 330 & 22 \\
\hline Brunei & 57 & 1.1 & 13 & $50 \%$ & 330 & 90 \\
\hline Bulgaria & 1347 & 1.1 & 68 & $100 \%$ & $120(30-90)$ & 104 \\
\hline Cambodia & 495 & 1.1 & 27 & $50 \%$ & 330 & 131 \\
\hline China & 50328 & 0.6 & 14,340 & $100 \%$ & $120(30-90)$ & 32 \\
\hline Colombia & 5286 & 1.3 & 324 & $50 \%$ & 330 & 28 \\
\hline Costa Rica & 136 & 1.1 & 62 & $50 \%$ & 330 & 45 \\
\hline Croatia & 277 & 1.3 & 60 & $50 \%$ & 330 & 75 \\
\hline Cyprus & 126 & 1.1 & 25 & $100 \%$ & $120(30-90)$ & 51 \\
\hline India & 32784 & 1.3 & 2,875 & $50 \%$ & 330 & 28 \\
\hline Indonesia & 7306 & 1.1 & 1,119 & $50 \%$ & 330 & 30 \\
\hline Hong Kong & 533 & 1.3 & 366 & $100 \%$ & $120(30-90)$ & 304 \\
\hline Kazakhstan & 3943 & 1.1 & 180 & $50 \%$ & 330 & 53 \\
\hline Malaysia & 1086 & 1.3 & 365 & $50 \%$ & 330 & 122 \\
\hline Malta & 70 & 1.1 & 15 & $100 \%$ & $120(30-90)$ & 68 \\
\hline Morocco & 2100 & 1.3 & 119 & $50 \%$ & 330 & 67 \\
\hline Peru & 943 & 1.1 & 227 & $50 \%$ & 330 & 40 \\
\hline Philippines & 2378 & 1.1 & 377 & $50 \%$ & 330 & 49 \\
\hline Romania & 1500 & 1.1 & 250 & $100 \%$ & $120(30-90)$ & 69 \\
\hline Russia & 17500 & 1.1 & 1,700 & $100 \%$ & $120(30-90)$ & 40 \\
\hline Saudi Arabia & 7813 & 1.1 & 793 & $50 \%$ & 330 & 52 \\
\hline Singapore & 650 & 1.2 & 372 & $100 \%$ & $120(30-90)$ & 202 \\
\hline South Africa & 2323 & 1.1 & 351 & $50 \%$ & 330 & 56 \\
\hline Taiwan & 6725 & 1.1 & 611 & $50 \%$ & 330 & 101 \\
\hline Thailand & 7241 & 1.1 & 544 & $50 \%$ & 330 & 89 \\
\hline Tunisia & 479 & 1.1 & 39 & $50 \%$ & 330 & 94 \\
\hline Vietnam & 251 & 1.1 & 262 & $50 \%$ & 330 & 198 \\
\hline ROW & 57225 & 1.1 & 7,276 & $50 \%$ & 330 & 48 \\
\hline
\end{tabular}

NoTES: This table reports the ICU capacities (see Table C.2 for details), estimated reproduction rates, GDP figures (obtained from World Development Indicators, 2019 current dollars), shared of population getting vaccine (for scenario 3), duration of vaccination days (for scenario 3) and openness index, which is defined as the ratio of imports and exports to GDP. 
Table C.2: ICU BED CAPACITIES

\begin{tabular}{|c|c|c|c|}
\hline ISO-3 & Country & ICU Covid-19 & Reference \\
\hline AUS & Australia & 1665 & https://www.mja.com.au/journal/2020/surge-capacity-australian-intensive-care-units-associated-covid-19-admissions \\
\hline AUT & Austria & 1000 & https://www.covid19healthsystem.org/countries/austria/livinghit.aspx?Section=2.1\%20Physical\%20infrastructure\&Type=Section \\
\hline BEL & Belgium & 2756 & https://www.covid19healthsystem.org/countries/belgium/livinghit.aspx?Section=2.1\%20Physical\%20infrastructure\&Type=Section \\
\hline CAN & Canada & 2713 & https://www.covid19healthsystem.org/countries/canada/livinghit.aspx?Section=2.1\%20Physical\%20infrastructure\&Type=Section \\
\hline CHL & Chile & 1383 & https://www.oecd.org/coronavirus/en/data-insights/intensive-care-beds-capacity \\
\hline CZE & Czech Republic & 4151 & https://www.covid19healthsystem.org/countries/czechrepublic/livinghit.aspx?Section=2.1\%20Physical\%20infrastructure\&Type=Section \\
\hline DNK & Denmark & 925 & https://www.sst.dk/-/media/Nyheder/2020/ITA_COVID_19_220320.ashx?la=da\&hash=633349284353F4D8559B231CDA64169D327F1227 \\
\hline EST & Estonia & 338 & https://www.ncbi.nlm.nih.gov/pmc/articles/PMC7472675/ \\
\hline FIN & Finland & 220 & https://www.covid19healthsystem.org/countries/finland/livinghit.aspx?Section=2.1\%20Physical\%20infrastructure\&Type=Section \\
\hline FRA & France & 8000 & https://www.covid19healthsystem.org/countries/france/livinghit.aspx?Section=2.1\%20Physical\%20infrastructure\&Type=Section \\
\hline DEU & Germany & 28000 & https://www.covid19healthsystem.org/countries/germany/livinghit.aspx?Section=2.1\%20Physical\%20infrastructure\&Type=Section \\
\hline GRC & Greece & 704 & https://www.covid19healthsystem.org/countries/greece/livinghit.aspx?Section=2.1\%20Physical\%20infrastructure\&Type=Section \\
\hline HUN & Hungary & 1094 & https://www.oecd.org/coronavirus/en/data-insights/intensive-care-beds-capacity \\
\hline ISL & Iceland & 163 & https://europepmc.org/article/med/32796182 \\
\hline IRL & Ireland & 248 & https://www.thejournal.ie/icu-bed-numbers-5217685-Sep2020/ \\
\hline ISR & Israel & 4900 & https://www.covid19healthsystem.org/countries/israel/livinghit.aspx?Section=2.1\%20Physical\%20infrastructure\&Type=Section \\
\hline ITA & Italy & 7700 & https://apnews.com/article/international-news-virus-outbreak-italy-barcelona-france-d7a43368a17f0abaff4d563151b84127 \\
\hline JPN & Japan & 3996 & https://journals.lww.com/ccmjournal/Fulltext/2020/05000/Critical_Care_Bed_Capacity_in_Asian_Countries_and.6.aspx \\
\hline KOR & Korea, Rep. & 5481 & https://journals.lww.com/ccmjournal/Fulltext/2020/05000/Critical_Care_Bed_Capacity_in_Asian_Countries_and.6.aspx \\
\hline LVA & Latvia & 186 & https://www.covid-19.no/critical-care-bed-numbers-in-europe \\
\hline LTU & Lithuania & 451 & https://www.ncbi.nlm.nih.gov/pmc/articles/PMC7472675/ \\
\hline LUX & Luxembourg & 91 & https://www.ncbi.nlm.nih.gov/pmc/articles/PMC7472675/ \\
\hline MEX & Mexico & 4211 & https://www.oecd.org/coronavirus/en/data-insights/intensive-care-beds-capacity \\
\hline NLD & Netherlands & 1161 & https://www.oecd.org/coronavirus/en/data-insights/intensive-care-beds-capacity \\
\hline NZL & New Zealand & 585 & https://www.nzherald.co.nz/nz/covid-19-coronavirus-new-zealands-intensive-care-unit-capacity-revealed/GYQ2FXOYHJECZAHU2YKHXYFW \\
\hline NOR & Norway & 455 & https://www.oecd.org/coronavirus/en/data-insights/intensive-care-beds-capacity \\
\hline POL & Poland & 3074 & https://www.ncbi.nlm.nih.gov/pmc/articles/PMC7472675/ \\
\hline PRT & Portugal & 455 & https://www.covid-19.no/critical-care-bed-numbers-in-europe \\
\hline SVK & Slovak Republic & 570 & https://www.ncbi.nlm.nih.gov/pmc/articles/PMC7472675/ \\
\hline SVN & Slovenia & 377 & https://www.ncbi.nlm.nih.gov/pmc/articles/PMC7472675/ \\
\hline ESP & Spain & 4566 & https://www.covid-19.no/critical-care-bed-numbers-in-europe \\
\hline SWE & Sweden & 365 & https://www.ncbi.nlm.nih.gov/pmc/articles/PMC7472675/ \\
\hline CHE & Switzerland & 1012 & https://www.oecd.org/coronavirus/en/data-insights/intensive-care-beds-capacity \\
\hline TUR & Turkey & 16850 & https://dosyasb.saglik.gov.tr/Eklenti/36164,siy2018en2pdf.pdf?0 \\
\hline GBR & United Kingdom & 7018 & https://www.oecd.org/coronavirus/en/data-insights/intensive-care-beds-capacity \\
\hline USA & United States & 84676 & https://www.oecd.org/coronavirus/en/data-insights/intensive-care-beds-capacity \\
\hline ARG & Argentina & 8404 & https://www.oecd-ilibrary.org/sites/63d94877-en/index.html?itemId=/content/component/63d94877-en \\
\hline BRA & Brazil & 43466 & https://www.oecd-ilibrary.org/sites/63d94877-en/index.html?itemId=/content/component/63d94877-en \\
\hline BRN & Brunei Darussalam & 57 & https://journals.lww.com/ccmjournal/Fulltext/2020/05000/Critical_Care_Bed_Capacity_in_Asian_Countries_and.6.aspx \\
\hline BGR & Bulgaria & 1347 & https://www.covid19healthsystem.org/countries/bulgaria/livinghit.aspx?Section=2.1\%20Physical\%20infrastructure\&Type=Section \\
\hline KHM & Cambodia & 495 & Selected to be close to the minimum observed levels. \\
\hline $\mathrm{CHN}$ & China & 50328 & https://journals.lww.com/ccmjournal/Fulltext/2020/05000/Critical_Care_Bed_Capacity_in_Asian_Countries_and.6.aspx \\
\hline $\mathrm{COL}$ & Colombia & 5286 & https://www.oecd-ilibrary.org/sites/63d94877-en/index.html?itemId=/content/component/63d94877-en \\
\hline CRI & Costa Rica & 136 & https://www.oecd-ilibrary.org/sites/63d94877-en/index.html?itemId=/content/component/63d94877-en \\
\hline HRV & Croatia & 277 & https://www.ncbi.nlm.nih.gov/pmc/articles/PMC7472675/ \\
\hline CYP & Cyprus & 126 & https://in-cyprus.philenews.com/coronavirus-seven-patients-in-intensive-care/ \\
\hline IND & India & 32784 & https://journals.lww.com/ccmjournal/Fulltext/2020/05000/Critical_Care_Bed_Capacity_in_Asian_Countries_and.6.aspx \\
\hline IDN & Indonesia & 7306 & https://journals.lww.com/ccmjournal/Fulltext/2020/05000/Critical_Care_Bed_Capacity_in_Asian_Countries_and.6.aspx \\
\hline HKG & Hong Kong SAR, China & 533 & https://journals.lww.com/ccmjournal/Fulltext/2020/05000/Critical_Care_Bed_Capacity_in_Asian_Countries_and.6.aspx \\
\hline KAZ & Kazakhstan & 3943 & https://journals.lww.com/ccmjournal/Fulltext/2020/05000/Critical_Care_Bed_Capacity_in_Asian_Countries_and.6.aspx \\
\hline MYS & Malaysia & 1086 & https://journals.lww.com/ccmjournal/Fulltext/2020/05000/Critical_Care_Bed_Capacity_in_Asian_Countries_and.6.aspx \\
\hline MLT & Malta & 70 & https://www.covid19healthsystem.org $/$ countries $/$ malta $/$ livinghit.aspx?Section $=2.1 \% 20 \mathrm{Physical} \% 20$ infrastructure\&Type $=$ Section \\
\hline MAR & Morocco & 2100 & https://northafricapost.com/39786-covid-19-morocco-expands-hospital-capacity.html \\
\hline PER & Peru & 943 & https://www.oecd-ilibrary.org/sites/63d94877-en/index.html?itemId=/content/component/63d94877-en \\
\hline PHL & Philippines & 2378 & https://journals.lww.com/ccmjournal/Fulltext/2020/05000/Critical_Care_Bed_Capacity_in_Asian_Countries_and.6.aspx \\
\hline ROU & Romania & 1500 & https://www.covid19healthsystem.org/countries/romania/livinghit.aspx?Section=2.1\%20Physical\%20infrastructure\&Type=Section \\
\hline RUS & Russian Federation & 17500 & https://tass.com/world/1162077 \\
\hline SAU & Saudi Arabia & 7813 & https://journals.lww.com/ccmjournal/Fulltext/2020/05000/Critical_Care_Bed_Capacity_in_Asian_Countries_and.6.aspx \\
\hline SGP & Singapore & 650 & https://journals.lww.com/ccmjournal/Fulltext/2020/05000/Critical_Care_Bed_Capacity_in_Asian_Countries_and.6.aspx \\
\hline ZAF & South Africa & 2323 & https://www.samrc.ac.za/news/covid-19-surge-investing-heavily-icu-capacity-not-only-option \\
\hline TWN & Taiwan & 6725 & https://journals.lww.com/ccmjournal/Fulltext/2020/05000/Critical_Care_Bed_Capacity_in_Asian_Countries_and.6.aspx \\
\hline THA & Thailand & 7241 & https://journals.lww.com/ccmjournal/Fulltext/2020/05000/Critical_Care_Bed_Capacity_in_Asian_Countries_and.6.aspx \\
\hline TUN & Tunisia & 479 & https://www.medrxiv.org/content/10.1101/2020.06.02.20120147v1.full.pdf \\
\hline VNM & Vietnam & 251 & https://www.who.int/docs/default-source/wpro---documents/countries/viet-nam/covid-19/vnm-moh-who-covid-19-sitrep4.pdf \\
\hline ROW & Rest of the World & 57225 & Selected to be close to the minimum observed levels. \\
\hline
\end{tabular}

NOTES: This table provides the resources from which we built the ICU capacities dedicated for Covid-19 patients in each country. If there is a direct number for the ICU beds for Covid-19 in a resource, we used that number. Otherwise we assigned 70\% of the total ICU beds to Covid-19 patients. We estimated this ratio from the countries that we have the information about dedicated ICU beds to Covid-19 patients. 
Table C.3: LIST OF ESSENTIAL SECTORS DURING LOCKDOWNS

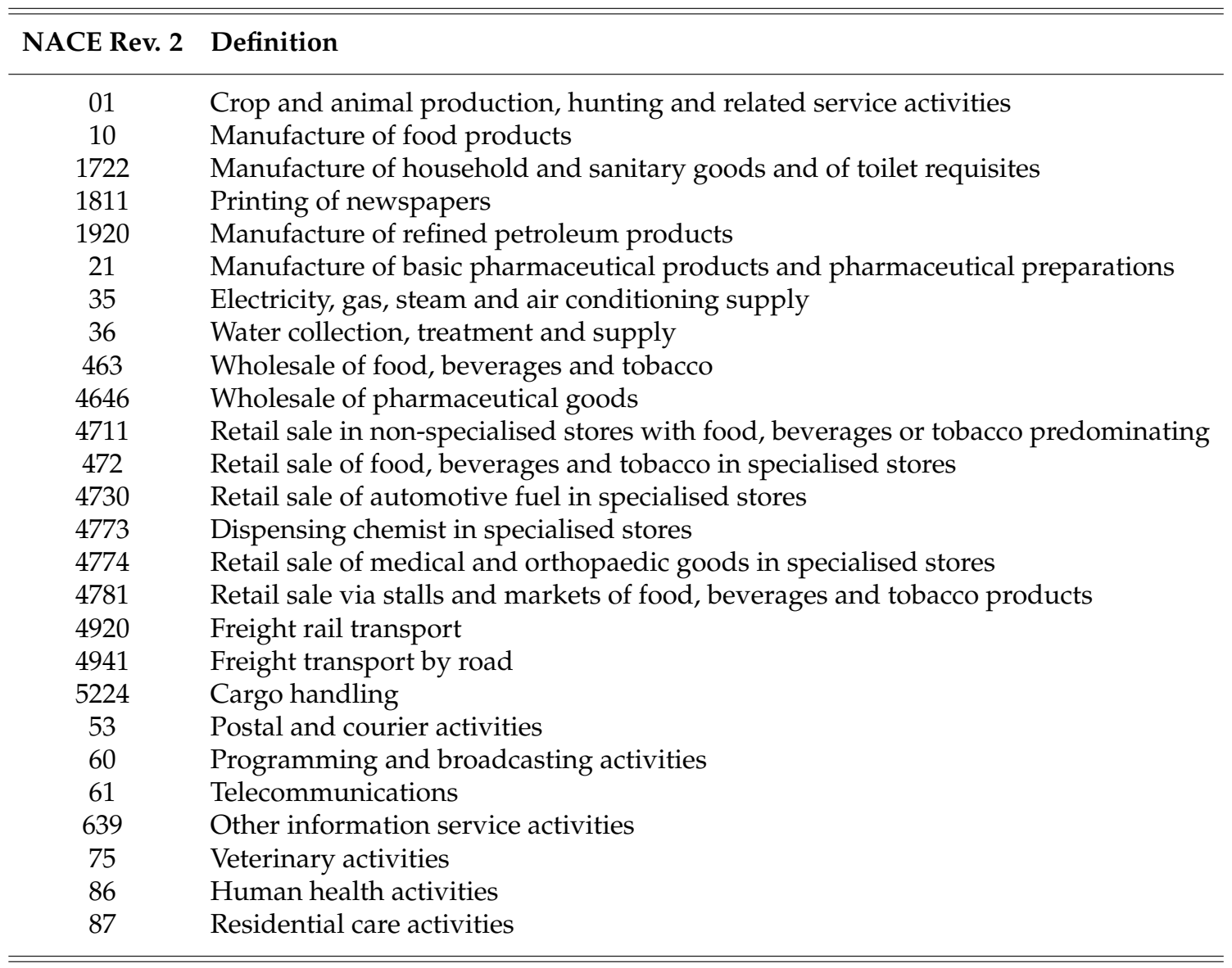

Notes: This table provides the list of the essential sectors that we consider for the implementation of lockdowns under Scenario I \& Scenario II. These sectors are identified by the full lockdown practices of countries. Turkish Ministry of Interior, for example, issued a decree on April 10, 2020 indicating the list of essential sectors. 


\section{Table C.4: MODEL-IMPliED CHANGES AGAINST DATA}

\begin{tabular}{lcc}
\hline \hline Dep. Var. & $\% \Delta \mathrm{Y}($ Model $)$ & $\% \Delta w_{f}$ (Model) \\
& $(1)$ & $(2)$ \\
\hline Input Shortage (Data) & $-0.2362^{* *}$ & $0.5448^{* *}$ \\
& $(0.086)$ & $(0.202)$ \\
Country FE & yes & yes \\
Obs. & 319 & 319 \\
$\mathrm{R}^{2}$ & 0.083 & 0.26 \\
\hline
\end{tabular}

NotES: Table C.4 presents the relationship between input shortages in the manufacturing sector of 21 European countries with the output changes $(\% \Delta \mathrm{Y})$ and factor price changes $\left(\% \Delta w_{f}\right)$ based on our model. We measure the severity of the input shortages using the responses of business managers collected by the EU Commission's Business and Consumer Survey. In the survey, we focus on the input shortage as a factor limiting production. Specifically, for any given quarter in the period 2003q1-2021q4, we quantify the severity of the input shortages in a given country-sector pair by the standard deviations above its country-specific average value over the period 2003q1-2021q4. As an additional robustness check to determine the relationship between input shortages and the actual data, we regress the percentage change in industrial production from December 2020 to December 2021 onto the change in input shortages observed in 2021q4 (relative to 2020q4). There is a negative and significant correlation of 0.12 between the severity of input shortages observed in the data and the decline in actual industrial production. Heteroskedastic-consistent standard errors are reported in parentheses. ${ }^{* * *}, * *$, and ${ }^{*}$ indicate significance at the $1 \%, 5 \%$, and $10 \%$ levels, respectively.

Table C.5: GDP Decline Relative to the Pre-Pandemic World (percent): No Endogenous Lockdowns

\begin{tabular}{ll|rrrc} 
& \multicolumn{3}{c}{ Baseline Scenarios } \\
\hline \hline & Parameter & World & AE & EMDE & $\begin{array}{l}\text { Share of } \\
\text { AEs (\%) }\end{array}$ \\
& Setup & 1.296 & 0.592 & 0.705 & 45.6 \\
\hline$(1)$ & $\xi_{i}^{\prime}, \xi_{i}=0.60$ (With lockdown) & 0.757 & 0.159 & 0.598 & 21.0 \\
$(2)$ & $\xi_{i}^{\prime}, \xi_{i}=0.60$ (Without Lockdown) & (Whot & \\
\hline \hline
\end{tabular}

NOTES: This table presents the total economic cost associated with the Covid-19 pandemic for the World, AEs, and EMDEs calculated under the scenario where lockdowns are not allowed. In this scenario, we assume that the pandemic is fully contained in AEs immediately. In EMDEs, the pandemic evolves at its natural course, but they could not impose any lockdown measures. We estimate the total economic cost of each of these scenarios under different trade elasticities set as 0.6 , which is the baseline scenario. $\xi$ denotes the trade elasticity for the production side, and $\xi^{\prime}$ denotes the trade elasticity for the consumption side; see Figure 7 for details. 


\section{Model Calculations}

\section{Notation and IO matrices}

Before moving into individual equations, let's define the variables and operations that we will use. The variables that we will use are summarized in Table D.1.

\section{Table D.1: NOTATION USED IN THE MODEL}

\begin{tabular}{l|l|l} 
Var. & Dimensions & Explanation \\
\hline$C$ & 1 & Number of countries. \\
$N(F)$ & 1 & Number of industries (factors). \\
$C N(C F)$ & 1 & Number of country-industry (country-factor) pairs. \\
$\Omega$ & $(C+C N+C F) \times(C+C N+C F)$ & Input shares (See Eq. D.1 for its structure). \\
$\Omega^{\mathcal{N}}$ & $C N \times C N$ & Intermediate input shares. \\
$\Omega^{\mathcal{N}}$ & $C N \times N$ & Sectoral aggregate input shares (calculated from $\left.\Omega^{\mathcal{N}}\right)$. \\
$\Omega^{\mathcal{F}}$ & $C N \times C F$ & Factor input shares. \\
$b \equiv \Omega^{0}$ & $C \times C N$ & Expenditure shares. \\
$b s \equiv \Omega^{0} S$ & $C \times N$ & Sectoral aggregate expenditure shares. \\
$\alpha$ & $C N \times 1$ & Sectoral value-added (VA) shares. \\
$\omega^{0}$ & $C \times N$ & Sectoral demand shocks. \\
$\Psi$ & $(C+C N+C F) \times(C+C N+C F)$ & Leontief Inverse: $(I-\Omega)$ (See Eq. D.2 for its structure). \\
$\Psi \mathcal{N}$ & $C N \times C N$ & Leontief Inverse for goods: $\left(I-\Omega^{\mathcal{N}}\right)^{-1}$. \\
$\Psi^{\mathcal{F}}$ & $C N \times C F$ & Leontief Inverse for factors: $\Psi \mathcal{N} \Omega^{\mathcal{F}}$. \\
$\lambda$ & $(C+C N+C F) \times 1$ & Domar weights. \\
$\lambda^{\mathcal{N}}$ & $C N \times 1$ & Domar weights for goods. \\
$\lambda^{\mathcal{F}}$ & $C F \times 1$ & Domar weights for factors. \\
$L$ & $C F \times 1$ & Factor size. \\
$\chi$ & $C \times 1$ & Expenditure/ Income shares of countries. \\
$p$ & $(C+C N+C F) \times 1$ & Prices (equals to wages for factors). \\
$w$ & $C F \times 1$ & Wages (price for factors). \\
$\mathcal{F}$ & $N \times 1$ & Elasticity of substitution (EoS) within sectors. \\
$\sigma$ & scalar & Consumption EoS across sectors. \\
$\mathcal{E}$ & scalar & EoS across input-bundles. \\
$\phi$ & scalar & EoS across VA and intermediate input bundle. \\
$1_{n}$ & $n$ & Vector of ones of dimension $n$. \\
$\mathbb{1}_{x}$ & $\operatorname{logical}$ & 1 if $x$ is true, 0 otherwise. \\
$I_{n}$ & $n \times n$ & Identity Matrix of size $n$. \\
\hline &
\end{tabular}

Here, we will explicitly show the structure of $\Omega$ and $\Psi$ matrices and their components. In particular, $\Omega$ matrix can be written as:

$$
\Omega=\left[\begin{array}{c|c|c}
0_{(C \times C)} & b=\Omega^{0} & 0_{(C \times C F)} \\
\hline 0_{(C N \times C)} & \Omega^{\mathcal{N}} & \Omega^{\mathcal{F}} \\
\hline 0_{(C F \times C)} & 0_{(C F \times C N)} & 0_{(C F \times C F)}
\end{array}\right]
$$


$\Omega^{\mathcal{N}}$ is $C N \times C N, \Omega^{\mathcal{F}}$ is $C N \times C F$ and $b$ is $C \times C N$ matrix. In our case, $\Omega^{\mathcal{F}}$ is a diagonal matrix whose diagonal elements are the value-added share in an industry. The corresponding $\Psi$ matrix is given by:

$$
\Psi=(I-\Omega)^{-1}=\left[\begin{array}{c|c|c}
I_{(C \times C)} & b \Psi \mathcal{N} & b \Psi \mathcal{N} \Omega^{\mathcal{F}} \\
\hline 0_{(C N \times C)} & \Psi \mathcal{N} & \Psi \mathcal{N} \Omega^{\mathcal{F}} \\
\hline 0_{(C F \times C)} & 0_{(C F \times C N)} & I_{(C F \times C F)}
\end{array}\right]
$$

Hence, if we just obtain $\Psi^{\mathcal{N}}$, the other parts of $\Psi$ matrix can be easily obtained.

\section{Solving for Shocks}

Let's denote the total expenditure in country $c$ with $E_{c} \equiv \sum_{i c^{\prime}} E_{i c^{\prime}}^{0 c}$ and its expenditure share in the world with $\chi_{c} \equiv E_{c} / E$. $C$ dimensional $\chi$ is the vectoral representation of the country shares. For expositional convenience and to highlight the difference between the consumption side and the production side, we will denote $b_{j m}^{c} \equiv \Omega_{j m}^{0 c}$. Hence, $b$ is a $C \times(C N+C F)$ matrix whose first $C N$ columns represent share of goods in final expenditure and last $C F$ columns are 0 . In this section, $(C N+C F) \times(C N+C F)$ dimensional $\Omega$ matrix represents the input-output relations between goods and factors only. For any $j m \in \mathcal{C N} \cup \mathcal{F}$, we can write:

$$
\begin{aligned}
\lambda_{j m} & =\frac{p_{j m} y_{j m}}{E} \\
& =\sum_{c \in \mathcal{C}} \frac{p_{j m} x_{j m}^{0 c}}{E_{c}} \frac{E_{c}}{E}+\sum_{k c} \frac{p_{j m} x_{j m}^{k c}}{E} \\
& =\sum_{c \in \mathcal{C}} b_{j m}^{c} \chi_{c}+\sum_{k c} \Omega_{j m}^{k c} \frac{p_{k c} y_{k c}}{E} \\
& =\sum_{c \in \mathcal{C}} b_{j m}^{c} \chi_{c}+\sum_{k c} \Omega_{j m}^{k c} \lambda_{k c} .
\end{aligned}
$$

In matrix notation:

$$
\lambda^{\prime}=\chi^{\prime} b+\lambda^{\prime} \Omega
$$

Inverting this equation yields:

$$
\lambda^{\prime}=\chi^{\prime} b \Psi
$$

$\Psi \equiv(I-\Omega)^{-1}$ is the $(N+F) \times(N+F)$ dimensional Leontief inverse matrix and does not include the final consumers. Taking the derivative of this equations yields:

$$
d \lambda^{\prime}=d \chi^{\prime} b \Psi+\chi^{\prime} d b \Psi+\chi^{\prime} b d \Psi .
$$


Since $\Psi=I+\Omega+\Omega^{2}+\Omega^{3}+\ldots$, we can write:

$$
\begin{aligned}
d \Psi & =d \Omega+\Omega d \Omega+d \Omega \Omega+\Omega^{2} d \Omega+\Omega d \Omega \Omega+d \Omega \Omega^{2}+\ldots \\
& =\left(I+\Omega+\Omega^{2}+\Omega^{3}+\ldots\right) d \Omega\left(I+\Omega+\Omega^{2}+\Omega^{3}+\ldots\right) \\
& =\Psi d \Omega \Psi .
\end{aligned}
$$

Alternatively:

$d\left(\Psi \Psi^{-1}\right)=0=d \Psi \Psi^{-1}+\Psi d \Psi^{-1} \Rightarrow d \Psi \Psi^{-1}=-\Psi d \Psi^{-1} \Rightarrow d \Psi=-\Psi d \Psi^{-1} \Psi=\Psi d \Omega \Psi$.

Therefore:

$$
\begin{aligned}
d \lambda^{\prime} & =d \chi^{\prime} b \Psi+\chi^{\prime} d b \Psi+\chi^{\prime} b d \Psi \\
& =d \chi^{\prime} b \Psi+\chi^{\prime} d b \Psi+\chi^{\prime} b \Psi d \Omega \Psi \\
& =d \chi^{\prime} b \Psi+\chi^{\prime} d b \Psi+\lambda^{\prime} d \Omega \Psi \\
& =\left(d \chi^{\prime} b+\chi^{\prime} d b+\lambda^{\prime} d \Omega\right) \Psi
\end{aligned}
$$

For the first term, we use the fact that $\chi_{c}=\sum_{f_{c}} \lambda_{f c}$. Hence:

$$
d \chi_{c}=\sum_{f c \in \mathcal{F}_{c}} d \lambda_{f_{c}}
$$

Our observed expenditure shocks are at the industry level. Let's denote a multiplicative expenditure shock to industry $j$ as $\omega_{j}^{0 c}$. Let's define industry $i^{\prime}$ s share in expenditure by consumers in country $c$ with $b s_{c j} \equiv \sum_{m} b_{j m}^{c}$. After the shock, sectoral expenditure shares change to $\omega_{j}^{0 c} b s_{c j}$. In the price index, this shock will be raised to power $\sigma$. From the consumption CES, we can write:

$$
\begin{aligned}
\frac{p_{j m} x_{j m}^{0 c}}{p_{0 c} Y_{c}}=\frac{p_{j m} x_{j m}^{0 c}}{E_{c}} & =b_{j m}^{c}\left(\omega_{j}^{0 c}\right)^{\sigma}\left(\frac{p_{j m}}{p_{j}^{0 c}}\right)^{-\xi_{i}}\left(\frac{p_{j}^{0 c}}{p_{0 c}}\right)^{-\sigma}\left(\frac{p_{j m}}{p_{0 c}}\right) \\
& =b_{j m}^{c}\left(\omega_{j}^{0 c}\right)^{\sigma}\left(p_{j m}\right)^{1-\xi_{i}}\left(p_{j}^{0 c}\right)^{\xi_{i}-\sigma}\left(p_{0 c}\right)^{\sigma-1} .
\end{aligned}
$$

We assume that we start with $\omega_{j}^{0 c}=1$. We can write the elements of $d b^{\prime}$ with:

$$
\begin{aligned}
d b_{j m}^{c} & =b_{j m}^{c} d \log b_{j m}^{c}=b_{j m}^{c} d \log \left(\frac{p_{j m} x_{j m}^{0 c}}{p_{0 c} E_{c}}\right) \\
& =b_{j m}^{c}\left(1-\xi_{j}\right) d \log p_{j m}+b_{j m}^{c}\left(\frac{\xi_{j}-\sigma}{b s_{c j}}\right) \sum_{v \in \mathcal{C}} b_{j v}^{c} d \log p_{j v} \\
& +b_{j m}^{c}(\sigma-1) \sum_{i v \in \mathcal{C} \mathcal{N}} b_{i v}^{c} d \log p_{i v}+b_{j m}^{c} \sigma d \log \omega_{j}^{0 c}
\end{aligned}
$$


For good production, corresponding input shares for goods are given by:

$$
\begin{aligned}
\frac{p_{j m} x_{j m}^{k c}}{p_{k c} y_{k c}} & =\Omega_{j m}^{k c}\left(\frac{p_{j m}}{p_{j}^{k c}}\right)^{-\xi_{i}}\left(\frac{p_{j}^{k c}}{p_{M}^{k c}}\right)^{-\varepsilon}\left(\frac{p_{M}^{k c}}{p_{k c}}\right)^{-\phi}\left(\frac{p_{j m}}{p_{k c}}\right) \\
& =\Omega_{j m}^{k c}\left(p_{j m}\right)^{1-\xi_{i}}\left(p_{j}^{k c}\right)^{\xi_{i}-\varepsilon}\left(p_{M_{k c}}\right)^{\varepsilon-\phi}\left(p_{k c}\right)^{\phi-1} .
\end{aligned}
$$

Let's define $\Omega s_{j}^{k c} \equiv \sum_{m} \Omega_{j m}^{k c}$ and the value added share as $\alpha_{k c} \equiv 1-\sum_{j m} \Omega_{j m}^{k c}$. We assume that the factors are industry-specific. Let's denote the factor used in industry $k c$ with $f_{k c} \equiv$ $k c+C N$. For that factor, $\Omega_{f_{k c}}^{k c}=\alpha_{k c}$. We can express the changes in non-zero elements of $\Omega$ :

$$
\begin{aligned}
d \Omega_{j m}^{k c} & =\Omega_{j m}^{k c} d \log \Omega_{j m}^{k c}=\Omega_{j m}^{k c} d \log \left(\frac{p_{j m} x_{j m}^{k c}}{p_{k c} y_{k c}}\right) \\
& =\Omega_{j m}^{k c}\left(1-\xi_{j}\right) d \log p_{j m}+\Omega_{j m}^{k c}\left(\frac{\xi_{j}-\varepsilon}{\Omega s_{j}^{k c}}\right) \sum_{v \in \mathcal{C}} \Omega_{j v}^{k c} d \log p_{j v} \\
& +\Omega_{j m}^{k c}\left(\frac{\varepsilon-\phi}{1-\alpha_{k c}}\right) \sum_{i v \in \mathcal{C N}} \Omega_{i v}^{k c} d \log p_{i v}+\Omega_{j m}^{k c}(\phi-1) d \log p_{k c} .
\end{aligned}
$$

Factor shares, on the other hand, are given by:

$$
\begin{aligned}
\frac{w_{f m} x_{j f}^{k c}}{p_{k c} y_{k c}} & =\Omega_{f m}^{k c}\left(\frac{w_{f m}}{p_{k c}}\right)^{-\phi}\left(\frac{w_{f m}}{p_{k c}}\right) \\
& =\Omega_{f m}^{k c}\left(w_{f m}\right)^{1-\phi}\left(p_{k c}\right)^{\phi-1} .
\end{aligned}
$$

We know that $\Omega_{f m}^{k c}$ is non-zero only if $f m=f_{k c}$. For this factor, we can write:

$$
\begin{aligned}
d \Omega_{f_{k c}}^{k c} & =\Omega_{f_{k c}}^{k c} d \log \Omega_{f_{k c}}^{k c}=\alpha_{k c} d \log \left(\frac{w_{f_{k c}} L_{f_{k c}}}{p_{k c} y_{k c}}\right) \\
& =\alpha_{k c}(1-\phi) d \log w_{f_{k c}}+\alpha_{k c}(\phi-1) d \log p_{k c} .
\end{aligned}
$$

$d \Omega_{f m}^{k c}=0$ for all other factors $f m \neq f_{k c}$.

\section{Sanity Check}

Since rows of $b$ matrix are normalized, $\sum_{j m \in \mathcal{C N}} d b_{j m}^{c}$ should be equal to 0 .

$$
\sum_{j m \in \mathcal{C} \mathcal{N}} d b_{j m}^{c}=\sum_{j m \in \mathcal{C N}} b_{j m}^{c}\left(1-\xi_{j}\right) d \log p_{j m}
$$




$$
\begin{aligned}
& +\sum_{j m \in \mathcal{C} \mathcal{N}} b_{j m}^{c}\left(\frac{\xi_{j}-\sigma}{b s_{c j}}\right) \sum_{v \in \mathcal{C}} b_{j v}^{c} d \log p_{j v} \\
& +\sum_{j m \in \mathcal{C} \mathcal{N}} b_{j m}^{c}(\sigma-1) \sum_{i v \in \mathcal{C} \mathcal{N}} b_{i v}^{c} d \log p_{i v} \\
& +\sum_{j m \in \mathcal{C} \mathcal{N}} b_{j m}^{c} \sigma d \log \omega_{j}^{0 c} .
\end{aligned}
$$

Rearranging the terms:

$$
\begin{aligned}
\sum_{j m \in \mathcal{C} \mathcal{N}} d b_{j m}^{c} & =\sum_{j m \in \mathcal{C} \mathcal{N}} b_{j m}^{c}\left(1-\xi_{j}\right) d \log p_{j m} \\
& +\sum_{j v \in \mathcal{C}} b_{j v}^{c} d \log p_{j v}\left(\frac{\xi_{j}-\sigma}{b s_{c j}}\right) \sum_{m \in \mathcal{C}} b_{j m}^{c} \\
& +\sum_{i v \in \mathcal{C} \mathcal{N}} b_{i v}^{c} d \log p_{i v}(\sigma-1) \sum_{j m \in \mathcal{C} \mathcal{N}} b_{j m}^{c} \\
& +\sum_{j m \in \mathcal{C} \mathcal{N}} b_{j m}^{c} \sigma d \log \omega_{j}^{0 c} .
\end{aligned}
$$

Since:

$$
\sum_{m \in \mathcal{C}} b_{j m}^{c}=b s_{c j} \quad \text { and } \quad \sum_{j m \in \mathcal{C} \mathcal{N}} b_{j m}^{c}=1
$$

We find that

$$
\begin{aligned}
\sum_{j m \in \mathcal{C} \mathcal{N}} d b_{j m}^{c} & =\sum_{j m \in \mathcal{C} \mathcal{N}} b_{j m}^{c} d \log p_{j m}\left[\left(1-\xi_{j}\right)+\left(\xi_{j}-\sigma\right)+(\sigma-1)\right] \\
& +\sum_{j m \in \mathcal{C} \mathcal{N}} b_{j m}^{c} \sigma d \log \omega_{j}^{0 c}=\sum_{j m \in \mathcal{C} \mathcal{N}} b_{j m}^{c} \sigma d \log \omega_{j}^{0 c}=0 .
\end{aligned}
$$

In last line, we use the fact that we normalize the shocks such that this term is zero.

Since rows of $\Omega$ are normalized, $\sum_{j m \in \mathcal{C N} \cup \mathcal{F}} d \Omega_{j m}^{k c}$ should be equal to 0 .

$$
\begin{aligned}
\sum_{j m \in \mathcal{C N} \cup \mathcal{F}} d \Omega_{j m}^{k c} & =\sum_{j m \in \mathcal{C} \mathcal{N}} \Omega_{j m}^{k c}\left(1-\xi_{j}\right) d \log p_{j m} \\
& +\sum_{j m \in \mathcal{C N}} \Omega_{j m}^{k c}\left(\frac{\xi_{j}-\varepsilon}{\Omega s_{j}^{k c}}\right) \sum_{v \in \mathcal{C}} \Omega_{j v}^{k c} d \log p_{j v} \\
& +\sum_{j m \in \mathcal{C N}} \Omega_{j m}^{k c}\left(\frac{\varepsilon-\phi}{1-\alpha_{k c}}\right) \sum_{i v \in \mathcal{C} \mathcal{N}} \Omega_{i v}^{k c} d \log p_{i v} \\
& +\sum_{j m \in \mathcal{C N}} \Omega_{j m}^{k c}(\phi-1) d \log p_{k c} \\
& +\alpha_{k c}(1-\phi) d \log w_{f_{k c}}+\alpha_{k c}(\phi-1) d \log p_{k c} .
\end{aligned}
$$


Rearranging the terms:

$$
\begin{aligned}
\sum_{j m \in \mathcal{C N} \cup \mathcal{F}} d \Omega_{j m}^{k c} & =\sum_{j m \in \mathcal{C N}} \Omega_{j m}^{k c}\left(1-\xi_{j}\right) d \log p_{j m} \\
& +\sum_{j v \in \mathcal{N}} \Omega_{j v}^{k c} d \log p_{j v}\left(\frac{\xi_{j}-\varepsilon}{\Omega s_{j}^{k c}}\right) \sum_{m \in \mathcal{C}} \Omega_{j m}^{k c} \\
& +\sum_{i v \in \mathcal{C} \mathcal{N}} \Omega_{i v}^{k c} d \log p_{i v}\left(\frac{\varepsilon-\phi}{1-\alpha_{k c}}\right) \sum_{j m \in \mathcal{C} \mathcal{N}} \Omega_{j m}^{k c} \\
& +\alpha_{k c}(1-\phi) d \log w_{f_{k c}}+(\phi-1) d \log p_{k c}\left(\sum_{j m \in \mathcal{C} \mathcal{N}} \Omega_{j m}^{k c}+\alpha_{k c}\right)
\end{aligned}
$$

Since:

$$
\sum_{m \in \mathcal{C}} \Omega_{j m}^{k c}=\Omega s_{j}^{k c} \quad \text { and } \quad \sum_{j m \in \mathcal{C N}} \Omega_{j m}^{k c}=1-\alpha_{k c}
$$

we can write:

$$
\begin{aligned}
\sum_{j m \in \mathcal{C N} \cup \mathcal{F}} d \Omega_{j m}^{k c} & =\sum_{j m \in \mathcal{C N}} \Omega_{j m}^{k c}\left[\left(1-\xi_{j}\right)+\left(\xi_{j}-\varepsilon\right)+(\varepsilon-\phi)\right] d \log p_{j m} \\
& +\alpha_{k c}(1-\phi) d \log w_{f_{k c}}+(\phi-1) d \log p_{k c} \\
& =(1-\phi)\left(\sum_{j m \in \mathcal{C} \mathcal{N}} \Omega_{j m}^{k c} d \log p_{j m}+\alpha_{k c} d \log w_{f_{k c}}-d \log p_{k c}\right) .
\end{aligned}
$$

By Shepard's Lemma:

$$
d \log p_{k c}=\sum_{j m \in \mathcal{C} \mathcal{N}} \Omega_{j m}^{k c} d \log p_{j m}+\alpha_{k c} d \log w_{f_{k c}} .
$$

Hence

$$
\sum_{j m \in \mathcal{C} \mathcal{N} \cup \mathcal{F}} d \Omega_{j m}^{k c}=0 .
$$

\section{Combining Differentials}

Recall:

$$
d \lambda^{\prime}=\left(d \chi^{\prime} b+\chi^{\prime} d b+\lambda^{\prime} d \Omega\right) \Psi
$$

. The first term in the parenthesis is:

$$
d \chi^{\prime} b=\sum_{c} \sum_{g \in F_{c}} d \lambda_{g} b_{j m}^{c} .
$$


Each element of the second term, $\chi^{\prime} d b$, is given by:

$$
\begin{aligned}
\sum_{c \in \mathcal{C}} \chi_{c} d b_{j m}^{c} & =\sum_{c \in \mathcal{C}} \chi_{c} b_{j m}^{c}\left(1-\xi_{j}\right) d \log p_{j m} \\
& +\sum_{c \in \mathcal{C}} \chi_{c} b_{j m}^{c}\left(\frac{\xi_{j}-\sigma}{b s_{c j}}\right) \sum_{v \in \mathcal{C}} b_{j v}^{c} d \log p_{j v} \\
& +\sum_{c \in \mathcal{C}} \chi_{c} b_{j m}^{c}(\sigma-1) \sum_{i v \in \mathcal{C N}} b_{i v}^{c} d \log p_{i v} \\
& +\sum_{c \in \mathcal{C}} \chi_{c} b_{j m}^{c} \sigma d \log \omega_{j}^{0 c} .
\end{aligned}
$$

We will separate the third term into two parts: one for the goods and one for the factors. For goods $k c \in \mathcal{N}:$

$$
\begin{aligned}
\sum_{k c} \lambda_{k c} d \Omega_{j m}^{k c} & =\sum_{k c} \lambda_{k c} \Omega_{j m}^{k c}\left(1-\xi_{j}\right) d \log p_{j m} \\
& +\sum_{k c} \lambda_{k c} \Omega_{j m}^{k c}\left(\frac{\xi_{j}-\varepsilon}{\Omega s_{j}^{k c}}\right) \sum_{v \in \mathcal{C}} \Omega_{j v}^{k c} d \log p_{j v} \\
& +\sum_{k c} \lambda_{k c} \Omega_{j m}^{k c}\left(\frac{\varepsilon-\phi}{1-\alpha_{k c}}\right) \sum_{i v \in \mathcal{C} \mathcal{N}} \Omega_{i v}^{k c} d \log p_{i v} \\
& +\sum_{k c} \lambda_{k c} \Omega_{j m}^{k c}(\phi-1) d \log p_{k c} .
\end{aligned}
$$

For the good $k c \in \mathcal{N}$ and factor $f_{k c}$ we have:

$$
\lambda^{\prime} d \Omega_{f_{k c}}^{k c}=\sum_{k c} \lambda_{k c} d \Omega_{f_{k c}}^{k c}=\sum_{k c} \lambda_{k c} \alpha_{k c}\left[(1-\phi) d \log w_{f_{k c}}+(\phi-1) d \log p_{k c}\right] .
$$

Combining these equations and using Shepard's Lemma to replace:

$$
d \log p_{j m}=\sum_{g \in \mathcal{F}} \Psi_{g}^{j m} d \log w_{g}
$$

and

$$
d \log \lambda_{g}=d \log w_{g}+d \log L_{g}
$$

we find that:

$$
\begin{aligned}
\frac{d \lambda_{f}}{\lambda_{f}} & =d \log \lambda_{f}=d \log w_{f}+d \log L_{f} \\
\text { Line 1: } & =\sum_{j m \in \mathcal{C} \mathcal{N}} \sum_{c \in \mathcal{C}} \sum_{g \in \mathcal{F}} \lambda_{g}\left[d \log w_{g}+d \log L_{g}\right] b_{j m}^{c} \mathbb{1}_{g \in \mathcal{F}_{c}} \Psi_{f}^{j m} / \lambda_{f} \\
\text { Line 2: } & \quad+\sum_{j m \in \mathcal{C} \mathcal{N}} \sum_{g \in \mathcal{F}} \sum_{c \in \mathcal{C}} \chi_{c} b_{j m}^{c}\left(1-\xi_{j}\right) \Psi_{g}^{j m} d \log w_{g} \Psi_{f}^{j m} / \lambda_{f}
\end{aligned}
$$




$$
\begin{array}{lll}
\text { Line 3: } & +\sum_{j m \in \mathcal{C} \mathcal{N}} \sum_{c \in \mathcal{C}} \sum_{v \in \mathcal{C}} \sum_{g \in \mathcal{F}} \chi_{c} b_{j m}^{c}\left(\frac{\xi_{j}-\sigma}{b s_{c j}}\right) b_{j v}^{c} \Psi_{g}^{j v} d \log w_{g} \Psi_{f}^{j m} / \lambda_{f} \\
\text { Line 4: } & +\sum_{j m \in \mathcal{C} \mathcal{N}} \sum_{c \in \mathcal{C}} \sum_{i v \in \mathcal{C} \mathcal{N}} \sum_{g \in \mathcal{F}} \chi_{c} b_{j m}^{c}(\sigma-1) b_{i v}^{c} \Psi_{g}^{i v} d \log w_{g} \Psi_{f}^{j m} / \lambda_{f} \\
\text { Line 5: } & +\sum_{j m \in \mathcal{C} \mathcal{N}} \sum_{c \in \mathcal{C}} \chi_{c} b_{j m}^{c} \sigma d \log \omega_{j}^{0 c} \Psi_{f}^{j m} / \lambda_{f} \\
\text { Line 6: } & +\sum_{j m \in \mathcal{C} \mathcal{N}} \sum_{k c \in \mathcal{C} \mathcal{N}} \sum_{g \in \mathcal{F}} \lambda_{k c} \Omega_{j m}^{k c}\left(1-\xi_{j}\right) \Psi_{g}^{j m} d \log w_{g} \Psi_{f}^{j m} / \lambda_{f} \\
\text { Line 7: } & +\sum_{j m \in \mathcal{C N}} \sum_{v \in \mathcal{C}} \sum_{k c \in \mathcal{C} \mathcal{N}} \sum_{g \in \mathcal{F}} \lambda_{k c} \Omega_{j m}^{k c}\left(\frac{\xi_{j}-\varepsilon}{\Omega s_{j}^{k c}}\right) \Omega_{j v}^{k c} \Psi_{g}^{j v} d \log w_{g} \Psi_{f}^{j m} / \lambda_{f} \\
\text { Line 8: } & +\sum_{j m \in \mathcal{C N N}} \sum_{k c \in \mathcal{C} \mathcal{N}} \sum_{i v \in \mathcal{C N}} \sum_{g \in \mathcal{F}} \lambda_{k c} \Omega_{j m}^{k c}\left(\frac{\varepsilon-\phi}{1-\alpha_{k c}}\right) \Omega_{i v}^{k c} \Psi_{g}^{i v} d \log w_{g} \Psi_{f}^{j m} / \lambda_{f} \\
\text { Line 9: } & +\sum_{j m \in \mathcal{C N N}} \sum_{k c \in \mathcal{C} \mathcal{N}} \sum_{g \in \mathcal{F}} \lambda_{k c} \Omega_{j m}^{k c}(\phi-1) \Psi_{g}^{k c} d \log w_{g} \Psi_{f}^{j m} / \lambda_{f} \\
\text { Line 10: } & +(1-\phi) d \log w_{f}+(\phi-1) \sum_{g \in \mathcal{F}} \Psi_{g}^{i i_{f}} d \log w_{g},
\end{array}
$$

where $i_{f}$ denotes the industry that uses the factor $f$. Last line derives from the fact that after multiplying with $\Psi^{\mathcal{F}}$ and dividing by $\lambda_{f}$ we obtain:

$$
\sum_{k c} \lambda_{k c} \alpha_{k c}\left[(1-\phi) d \log w_{f_{k c}}+(\phi-1) d \log p_{k c}\right] \Psi_{f}^{f_{k c}} / \lambda_{f}
$$

$\operatorname{But} \Psi_{f}^{f_{k c}}=1$ if $f=f_{k c}$ and 0 otherwise. Hence, the only surviving term is for good $k c=i_{f}$.

$$
\lambda_{i_{f}} \alpha_{i_{f}} / \lambda_{f}\left[(1-\phi) d \log w_{f}+(\phi-1) d \log p_{i_{f}}\right] .
$$

Note that $\lambda_{i_{f}} \alpha_{i_{f}}=\lambda_{f}$. Replacing $d \log p_{i_{f}}=\sum_{g \in \mathcal{F}} \Psi_{g}^{i_{f}} d \log w_{g}$ gives us the last line.

\section{Model solution with matrix operations}

Special Matrix Operations. There are also several special matrix operations that we use in our computation. $\odot$ represent element-by-element multiplication and $\oslash$ represent elementby-element division. When these operations are between matrices of different sizes, we will assume that the smaller dimensional matrix would be repeated to match the larger dimensional matrix. Furthermore, when we use a logic operator with a matrix, it implies an element-by-element binarization. So $M>0$ will return a binary matrix of the same size of $M$ with elements equal to 1 if the corresponding element in $M$ is larger than 0 . Lastly, we denote the tensor operator with $\otimes$. 
Solution Strategy. Our ultimate goal is to transform equations in the previous section above to the format:

$$
d \log w=A d \log w+B
$$

type equation, so we can solve $d \log w$, which will give us $d \log p$, which in turn will give us $d \log \Omega$. A matrix is $C F \times C F$ matrix and $B$ is a $C F$ dimensional vector. $A$ matrix will multiply a vector of $d \log w_{g}$ to transform into a $d \log w_{f}$. we can now write the contributions of each line of $d \log w$ equations separately. Each line will be decomposed into:

$$
A^{(l)} d \log w+B^{(l)}
$$

matrices where $l$ represents line numbers. Each component of $A_{f, g}^{(l)}$ will be mapping $d \log w_{g}$ to $d \log w_{f}$. Combining all these lines, we can write:

$$
A=\sum_{l=1}^{10} A^{(l)} \quad \text { and } \quad B=\sum_{l=1}^{10} B^{(l)}
$$

\section{Line 1:}

$$
\sum_{j m \in \mathcal{C} \mathcal{N}} \sum_{c \in \mathcal{C}} \sum_{g \in \mathcal{F}} \lambda_{g}\left[d \log w_{g}+d \log L_{g}\right] b_{j m}^{c} \mathbb{1}_{g \in \mathcal{F}_{c}} \Psi_{f}^{j m} / \lambda_{f}
$$

Hence:

$$
A_{f, g}^{(1)}=\sum_{j m \in \mathcal{C} \mathcal{N}} \sum_{c \in \mathcal{C}} b_{j m}^{c} \lambda_{g} \mathbb{1}_{g \in \mathcal{F}_{c}} \Psi_{f}^{j m} / \lambda_{f}
$$

and

$$
B^{(1)}=A^{(1)} d \log L .
$$

We can turn $\mathbb{1}_{g \in \mathcal{F}_{c}}$ into a binary $C F \times C$ matrix whose $(g, c)$ th element is 1 iff $g \in \mathcal{F}_{c}$ by $I_{C} \otimes 1_{F}$. Hence:

$$
A^{(1)}=\left(\left(\Psi^{\mathcal{F}}\right)^{\prime} \oslash \lambda^{\mathcal{F}}\right)\left[b^{\prime}\left(\left(I_{C} \otimes 1_{F}^{\prime}\right) \odot\left(\lambda^{\mathcal{F}}\right)^{\prime}\right)\right]
$$

Line 2:

$$
\begin{gathered}
\sum_{j m \in \mathcal{C} \mathcal{N}} \sum_{g \in \mathcal{F}} \sum_{c \in \mathcal{C}} \chi_{c} b_{j m}^{c}\left(1-\xi_{j}\right) \Psi_{g}^{j m} d \log w_{g} \Psi_{f}^{j m} / \lambda_{f} \\
A_{f, g}^{(2)}=\sum_{j m \in \mathcal{C} \mathcal{N}} \sum_{c \in \mathcal{C}} \chi_{c} b_{j m}^{c}\left(1-\xi_{j}\right) \Psi_{g}^{j m} \Psi_{f}^{j m} / \lambda_{f} \\
A^{(2)}=\left(\left(\Psi^{\mathcal{F}}\right)^{\prime} \oslash \lambda^{\mathcal{F}}\right)\left(\Psi^{\mathcal{F}} \odot\left[1_{C} \otimes(1-\xi)\right] \odot\left[b^{\prime} \chi\right]\right)
\end{gathered}
$$

Line 3:

$$
\sum_{j m \in \mathcal{C} N} \sum_{c \in \mathcal{C}} \sum_{v \in \mathcal{C}} \sum_{g \in \mathcal{F}} \chi_{c} b_{j m}^{c}\left(\frac{\xi_{j}-\sigma}{b s_{c j}}\right) b_{j v}^{c} \Psi_{g}^{j v} d \log w_{g} \Psi_{f}^{j m} / \lambda_{f}
$$




$$
\begin{gathered}
A_{f, g}^{(3)}=\sum_{j \in \mathcal{I}}\left(\xi_{j}-\sigma\right) \sum_{m \in \mathcal{C}}\left(\Psi_{f}^{j m} / \lambda_{f}\right) \sum_{c \in \mathcal{C}}\left(\frac{\chi_{c} b_{j m}^{c}}{b s_{c j}}\right) \sum_{v \in \mathcal{C}} b_{j v}^{c} \Psi_{g}^{j v} \\
B^{(3)}=0_{C F} .
\end{gathered}
$$

With these definitions, we can write:

$$
A^{(3)}=\sum_{j \in \mathcal{I}}\left(\xi_{j}-\sigma\right)\left(\left(\Psi_{(j)}^{\mathcal{F}}\right)^{\prime} \oslash \lambda^{\mathcal{F}}\right)\left(\left[\chi \odot b_{(j)} \oslash b s_{(j)}\right]^{\prime} b_{(j)}\right) \Psi_{(j)}^{\mathcal{F}}
$$

where $(j)$ indexes the row or columns corresponding to industry $j$.

\section{Line 4:}

$$
\begin{gathered}
\sum_{j m \in \mathcal{C} \mathcal{N}} \sum_{c \in \mathcal{C}} \sum_{i v \in \mathcal{C} \mathcal{N}} \sum_{g \in \mathcal{F}} \chi_{c} b_{j m}^{c}(\sigma-1) b_{i v}^{c} \Psi_{g}^{i v} d \log w_{g} \Psi_{f}^{j m} / \lambda_{f} \\
A_{f, g}^{(4)}=(\sigma-1) \sum_{j m \in \mathcal{C} \mathcal{N}}\left(\Psi_{f}^{j m} / \lambda_{f}\right) \sum_{c \in \mathcal{C}} \chi_{c} b_{j m}^{c} \sum_{i v \in \mathcal{C} \mathcal{N}} b_{i v}^{c} \Psi_{g}^{i v} \\
B^{(4)}=0_{C F} . \\
A^{(4)}=(\sigma-1)\left(\left(\Psi^{\mathcal{F}}\right)^{\prime} \oslash \lambda^{\mathcal{F}}\right)(\chi \odot b)^{\prime} b \Psi^{\mathcal{F}}
\end{gathered}
$$

\section{Line 5:}

$$
\begin{gathered}
\sum_{j m \in \mathcal{C} \mathcal{N}} \sum_{c \in \mathcal{C}} \chi_{c} b_{j m}^{c} \sigma d \log \omega_{j}^{0 c} \Psi_{f}^{j m} / \lambda_{f}=B_{f}^{(5)} \\
A^{(5)}=0_{C F \times C F} \\
B^{(5)}=\sigma\left(\left(\Psi^{\mathcal{F}}\right)^{\prime} \oslash \lambda^{\mathcal{F}}\right)\left[(b \odot d \log \omega)^{\prime} \chi\right]
\end{gathered}
$$

Line 6:

$$
\begin{gathered}
\sum_{j m \in \mathcal{C} \mathcal{N}} \sum_{k c \in \mathcal{C} \mathcal{N}} \sum_{g \in \mathcal{F}} \lambda_{k c}\left(1-\xi_{j}\right) \mathbb{1}_{\Omega_{j m}^{k c}>0} \Psi_{g}^{j m} d \log w_{g} \Psi_{f}^{j m} / \lambda_{f} \\
A_{f, g}^{(6)}=\sum_{j m \in \mathcal{C} \mathcal{N}} \sum_{k c \in \mathcal{C} \mathcal{N}} \lambda_{k c} \Omega_{j m}^{k c}\left(1-\xi_{j}\right) \Psi_{g}^{j m} \Psi_{f}^{j m} / \lambda_{f} \\
B^{(6)}=0_{C F} . \\
A^{(6)}=\left(\left(\Psi^{\mathcal{F}}\right)^{\prime} \oslash \lambda^{\mathcal{F}}\right)\left(\Psi^{\mathcal{F}} \odot\left[1_{C} \otimes(1-\xi)\right] \odot\left[\left(\Omega^{\mathcal{N}}\right)^{\prime} \lambda^{\mathcal{N}}\right]\right)
\end{gathered}
$$

\section{Line 7:}

$$
\begin{gathered}
\sum_{j m \in \mathcal{C} \mathcal{N}} \sum_{v \in \mathcal{C}} \sum_{k c \in \mathcal{C} \mathcal{N}} \sum_{g \in \mathcal{F}} \lambda_{k c} \Omega_{j m}^{k c}\left(\frac{\xi_{j}-\varepsilon}{\Omega s_{j}^{k c}}\right) \Omega_{j v}^{k c} \Psi_{g}^{j v} d \log w_{g} \Psi_{f}^{j m} / \lambda_{f} \\
A_{f, g}^{(7)}=\sum_{j \in \mathcal{I}} \sum_{k c \in \mathcal{C} \mathcal{N}} \sum_{m \in \mathcal{C}}\left(\Psi_{f}^{j m} / \lambda_{f}\right) \lambda_{k c} \Omega_{j m}^{k c}\left(\frac{\xi_{j}-\varepsilon}{\Omega s_{j}^{k c}}\right) \sum_{v \in \mathcal{C}} \Omega_{j v}^{k c} \Psi_{g}^{j v} \\
B^{(7)}=0_{C F} .
\end{gathered}
$$




$$
A^{(7)}=\sum_{j \in \mathcal{I}}\left(\xi_{j}-\sigma\right)\left(\left(\Psi_{(j)}^{\mathcal{F}}\right)^{\prime} \oslash \lambda^{\mathcal{F}}\right)\left(\left[\chi \odot \Omega_{(j)}^{\mathcal{N}} \oslash \Omega s_{(j)}\right]^{\prime} \Omega_{(j)}^{\mathcal{N}}\right) \Psi_{(j)}^{\mathcal{F}}
$$

\section{Line 8:}

$$
\begin{gathered}
\sum_{j m \in \mathcal{C} \mathcal{N}} \sum_{k c \in \mathcal{C} \mathcal{N}} \sum_{i v \in \mathcal{C} \mathcal{N}} \sum_{g \in \mathcal{F}} \lambda_{k c} \Omega_{j m}^{k c}\left(\frac{\varepsilon-\phi}{1-\alpha_{k c}}\right) \Omega_{i v}^{k c} \Psi_{g}^{i v} d \log w_{g} \Psi_{f}^{j m} / \lambda_{f} \\
A_{f, g}^{(8)}=\sum_{j m \in \mathcal{C} \mathcal{N}} \Psi_{f}^{j m} / \lambda_{f} \sum_{k c \in \mathcal{C} \mathcal{N}} \lambda_{k c} \Omega_{j m}^{k c}\left(\frac{\varepsilon-\phi}{1-\alpha_{k c}}\right) \sum_{i v \in \mathcal{C} \mathcal{N}} \Omega_{i v}^{k c} \Psi_{g}^{i v} \\
B^{(8)}=0_{C F} . \\
A^{(8)}=(\varepsilon-\phi)\left(\left(\Psi^{\mathcal{F}}\right)^{\prime} \oslash \lambda^{\mathcal{F}}\right)\left(\left[\lambda^{\mathcal{N}} \oslash(1-\alpha)\right] \odot \Omega^{\mathcal{N}}\right)^{\prime} \Omega^{\mathcal{N}} \Psi^{\mathcal{F}}
\end{gathered}
$$

\section{Line 9:}

$$
\begin{gathered}
\sum_{j m \in \mathcal{C} N} \sum_{k c \in \mathcal{C} \mathcal{N}} \sum_{g \in \mathcal{F}} \lambda_{k c} \Omega_{j m}^{k c} \Psi_{g}^{k c} d \log w_{g} \Psi_{f}^{j m} / \lambda_{f} \\
A_{f, g}^{(9)}=(\phi-1) \sum_{j m \in \mathcal{C} \mathcal{N}}\left(\Psi_{f}^{j m} / \lambda_{f}\right) \sum_{k c \in \mathcal{C} \mathcal{N}} \lambda_{k c} \Omega_{j m}^{k c} \Psi_{g}^{k c} \\
B^{(9)}=0_{C F} . \\
A^{(9)}=(\phi-1)\left(\left(\Psi^{\mathcal{F}}\right)^{\prime} \oslash \lambda^{\mathcal{F}}\right)\left(\Omega^{\mathcal{N}} \odot \lambda^{\mathcal{N}}\right)^{\prime} \Psi^{\mathcal{F}}
\end{gathered}
$$

Line 10:

$$
\begin{gathered}
(1-\phi) d \log w_{f}+(\phi-1) \sum_{g \in \mathcal{F}} \Psi_{g}^{i_{f}} d \log w_{g} \\
A_{f, g}^{(10)}=(1-\phi) \mathbb{1}_{f=g}+(\phi-1) \Psi_{g}^{i_{f}} \\
B^{(10)}=0_{C F} . \\
A^{(10)}=(1-\phi) I_{C F}+(\phi-1) \Psi^{\mathcal{F}}=(1-\phi)\left(I_{C F}-\Psi^{\mathcal{F}}\right)
\end{gathered}
$$

\section{Sanity Check}

If all the calculations are correct, the resultant $A$ matrix should be singular because of redundancies. Therefore, we need to replace one of the conditions with the fact that the world nominal GDP is constant:

$$
d E=\sum_{g} d \lambda_{g}=\sum_{g} \lambda_{g}\left(d \log w_{g}+d \log L_{g}\right)=0 .
$$

Hence, we can use this relation to break the singularity:

$$
A_{1,1}=0, \quad A_{1, g>1}=-\lambda_{g} \quad \text { and } \quad B_{1}=-\log L^{\prime} \cdot \lambda^{\mathcal{F}} .
$$


Table D.2: Formulating $d \log w=A d \log w+B$

\begin{tabular}{|c|c|c|c|}
\hline \# & Equation & $A$ & $B$ \\
\hline 1 & $\sum_{j m \in \mathcal{C N}} \sum_{c \in \mathcal{C}} \sum_{g \in \mathcal{F}} \lambda_{g}\left[d \log w_{g}+d \log L_{g}\right] b_{j m}^{c} \mathbb{1}_{g \in \mathcal{F}_{c}} \Psi_{f}^{j m} / \lambda_{f}$ & $\left(\left(\Psi^{\mathcal{F}}\right)^{\prime} \oslash \lambda^{\mathcal{F}}\right)\left[b^{\prime}\left(\left(I_{C} \otimes 1_{F}^{\prime}\right) \odot\left(\lambda^{\mathcal{F}}\right)^{\prime}\right)\right]$ & $A^{(1)} d \log L$ \\
\hline 2 & $\sum_{j m \in \mathcal{C N N}} \sum_{g \in \mathcal{F}} \sum_{c \in \mathcal{C}} \chi_{c} b_{j m}^{c}\left(1-\xi_{j}\right) \Psi_{g}^{j m} d \log w_{g} \Psi_{f}^{j m} / \lambda_{f}$ & $\left(\left(\Psi^{\mathcal{F}}\right)^{\prime} \oslash \lambda^{\mathcal{F}}\right)\left(\Psi^{\mathcal{F}} \odot\left[1_{C} \otimes(1-\xi)\right] \odot\left[b^{\prime} \chi\right]\right)$ & $0_{C F}$ \\
\hline 3 & $\sum_{j m \in \mathcal{C N}} \sum_{c \in \mathcal{C}} \sum_{v \in \mathcal{C}} \sum_{g \in \mathcal{F}} \chi_{c} b_{j m}^{c}\left(\frac{\xi_{j}-\sigma}{b s_{c j}}\right) b_{j v}^{c} \Psi_{g}^{j v} d \log w_{g} \Psi_{f}^{j m} / \lambda_{f}$ & $\sum_{j \in \mathcal{I}}\left(\xi_{j}-\sigma\right)\left(\left(\Psi_{(j)}^{\mathcal{F}}\right)^{\prime} \oslash \lambda^{\mathcal{F}}\right)\left(\left[\chi \odot b_{(j)} \oslash b s_{(j)}\right]^{\prime} b_{(j)}\right) \Psi_{(j)}^{\mathcal{F}}$ & $0_{C F}$ \\
\hline 4 & $\sum_{j m \in \mathcal{C N N}} \sum_{c \in \mathcal{C}} \sum_{i v \in \mathcal{C N}} \sum_{g \in \mathcal{F}} \chi_{c} b_{j m}^{c}(\sigma-1) b_{i v}^{c} \Psi_{g}^{i v} d \log w_{g} \Psi_{f}^{j m} / \lambda_{f}$ & $(\sigma-1)\left(\left(\Psi^{\mathcal{F}}\right)^{\prime} \oslash \lambda^{\mathcal{F}}\right)(\chi \odot b)^{\prime} b \Psi^{\mathcal{F}}$ & $0_{C F}$ \\
\hline 5 & $\sum_{j m \in \mathcal{C N}} \sum_{c \in \mathcal{C}} \chi_{c} b_{j m}^{c} \sigma d \log \omega_{j}^{0 c} \Psi_{f}^{j m} / \lambda_{f}$ & $0_{C F \times C F}$ & $\sigma\left(\left(\Psi^{\mathcal{F}}\right)^{\prime} \oslash \lambda^{\mathcal{F}}\right)\left[\chi^{\prime}(b \odot d \log \omega)\right]^{\prime}$ \\
\hline 6 & $\sum_{j m \in \mathcal{C N}} \sum_{k c \in \mathcal{C N}} \sum_{g \in \mathcal{F}} \lambda_{k c} \Omega_{j m}^{k c}\left(1-\xi_{j}\right) \Psi_{g}^{j m} d \log w_{g} \Psi_{f}^{j m} / \lambda_{f}$ & $\left(\left(\Psi^{\mathcal{F}}\right)^{\prime} \oslash \lambda^{\mathcal{F}}\right)\left(\Psi^{\mathcal{F}} \odot\left[1_{C} \otimes\left(1-\xi^{\mathcal{Z}}\right)\right] \odot\left[\left(\Omega^{\mathcal{N}}\right)^{\prime} \lambda^{\mathcal{N}}\right]\right)$ & $0_{C F}$ \\
\hline 7 & $\sum_{j m \in \mathcal{C N}} \sum_{v \in \mathcal{C}} \sum_{k c \in \mathcal{C N}} \sum_{g \in \mathcal{F}} \lambda_{k c} \Omega_{j m}^{k c}\left(\frac{\tilde{\xi}_{j}-\varepsilon}{\Omega_{j}^{k c}}\right) \Omega_{j v}^{k c} \Psi_{g}^{j v} d \log w_{g} \Psi_{f}^{j m} / \lambda_{f}$ & $\sum_{j \in \mathcal{I}}\left(\xi_{j}-\sigma\right)\left(\left(\Psi_{(j)}^{\mathcal{F}}\right)^{\prime} \oslash \lambda^{\mathcal{F}}\right)\left(\left[\chi \odot \Omega_{(j)}^{\mathcal{N}} \oslash \Omega_{(j)}\right]^{\prime} \Omega_{(j)}^{\mathcal{N}}\right) \Psi_{(j)}^{\mathcal{F}}$ & $0_{C F}$ \\
\hline 8 & $\sum_{j m \in \mathcal{C N}} \sum_{k c \in \mathcal{C N}} \sum_{i v \in \mathcal{C N N}} \sum_{g \in \mathcal{F}} \lambda_{k c} \Omega_{j m}^{k c}\left(\frac{\varepsilon-\phi}{1-\alpha_{k c}}\right) \Omega_{i v}^{k c} \Psi_{g}^{i v} d \log w_{g} \Psi_{f}^{j m} / \lambda_{f}$ & $(\varepsilon-\phi)\left(\left(\Psi^{\mathcal{F}}\right)^{\prime} \oslash \lambda^{\mathcal{F}}\right)\left(\left[\lambda^{\mathcal{N}} \oslash(1-\alpha)\right] \odot \Omega^{\mathcal{N}}\right)^{\prime} \Omega^{\mathcal{N}} \Psi^{\mathcal{F}}$ & $0_{C F}$ \\
\hline 9 & $\sum_{j m \in \mathcal{C N}} \sum_{k c \in \mathcal{C N}} \sum_{g \in \mathcal{F}} \lambda_{k c} \Omega_{j m}^{k c} \Psi_{g}^{k c} d \log w_{g} \Psi_{f}^{j m} / \lambda_{f}$ & $(\phi-1)\left(\left(\Psi^{\mathcal{F}}\right)^{\prime} \oslash \lambda^{\mathcal{F}}\right)\left(\Omega^{\mathcal{N}} \odot \lambda^{\mathcal{N}}\right)^{\prime} \Psi^{\mathcal{F}}$ & $0_{C F}$ \\
\hline 10 & $(\phi-1) \sum_{g \in \mathcal{F}} \Psi_{g}^{i_{f}} d \log w_{g}$ & $(1-\phi)\left(I_{C F}-\Psi^{\mathcal{F}}\right)$ & $0_{C F}$ \\
\hline & $\sum_{g} d \lambda_{g}=\sum_{g} \lambda_{g}\left(d \log w_{g}+d\right.$ & $\mid \gamma 1,1$ & \\
\hline
\end{tabular}

\section{Updating Variables}

After solving for $d \log w_{g}$, we can solve for other variables as follows.

Good Prices: Using Shepard's Lemma, we can easily obtain good prices with:

$$
d \log p_{j m}=\sum_{g \in \mathcal{F}} \Psi_{g}^{j m} d \log w_{g}
$$

Factor Domar Weights are related to the factor wages with:

$$
d \lambda_{f}=\lambda_{f}\left(d \log w_{f}+d \log L_{f}\right)
$$

Country Income Shares:

$$
d \chi_{c}=\sum_{f \in \mathcal{F}_{c}} d \lambda_{f}
$$

\section{Changes in consumption patterns:}

$$
\begin{aligned}
d b_{j m}^{c} & =b_{j m}^{c}\left(1-\xi_{j}\right) d \log p_{j m}+b_{j m}^{c}\left(\frac{\xi_{j}-\sigma}{b s_{c j}}\right) \sum_{v \in \mathcal{C}} b_{j v}^{c} d \log p_{j v} \\
& +b_{j m}^{c}(\sigma-1) \sum_{i v \in \mathcal{C} \mathcal{N}} b_{i v}^{c} d \log p_{i v}+b_{j m}^{c} \sigma d \log \omega_{j}^{0 c}
\end{aligned}
$$

Here, all the terms have $b_{j m}^{c}$ as a multiplier. That amounts to element by element multiplication. Ignoring that term, we will calculate the rest of the terms which will constitute a $C \times C N$ matrix, which we will denote with $B T^{t}$ where $t$ stands for term.:

\section{Term 1:}

$$
\left(1-\xi_{j}\right) d \log p_{j m}
$$


In Matrix notation:

$$
B T^{1}=1_{C} \otimes\left(\left[1_{C} \otimes(1-\xi)\right] \odot d \log p\right)^{\prime}
$$

Term 2:

$$
\left(\frac{\xi_{j}-\sigma}{b s_{c j}}\right) \sum_{v \in \mathcal{C}} b_{j v}^{c} d \log p_{j v}
$$

In Matrix notation:

$$
B T_{(j)}^{2}=1_{C}^{\prime} \otimes\left[\left(\xi_{j}-\sigma\right)\left(b_{(j)} \oslash b s_{(j)}\right) d \log p_{(j)}\right]
$$

Term 3:

$$
(\sigma-1) \sum_{i v \in \mathcal{C} \mathcal{N}} b_{i v}^{c} d \log p_{i v}
$$

In Matrix notation:

$$
B T^{3}=(\sigma-1) 1_{C N}^{\prime} \otimes(b d \log p)
$$

Term 4:

$$
\sigma d \log \omega_{j}^{0 c}
$$

In Matrix notation:

$$
B T^{4}=\sigma d \log \omega
$$

\section{Changes in input weights:}

$$
\begin{aligned}
d \Omega_{j m}^{k c} & =\Omega_{j m}^{k c}\left(1-\xi_{j}\right) d \log p_{j m}+\Omega_{j m}^{k c}\left(\frac{\xi_{j}-\varepsilon}{\Omega s_{j}^{k c}}\right) \sum_{v \in \mathcal{C}} \Omega_{j v}^{k c} d \log p_{j v} \\
& +\Omega_{j m}^{k c}\left(\frac{\varepsilon-\phi}{1-\alpha_{k c}}\right) \sum_{i v \in \mathcal{C N}} \Omega_{i v}^{k c} d \log p_{i v}+\Omega_{j m}^{k c}(\phi-1) d \log p_{k c}
\end{aligned}
$$

Here, all the terms have $\Omega_{j m}^{k c}$ as a multiplier. That amounts to element by element multiplication. Ignoring that term, we will calculate the rest of the terms which will constitute a $C N \times C N$ matrix, which we will denote with $O T^{t}$ where $t$ stands for term.:

\section{Term 1:}

$$
\left(1-\xi_{j}\right) d \log p_{j m}
$$

In Matrix notation:

$$
O T^{1}=1_{C N} \otimes\left(\left[1_{C} \otimes(1-\xi)\right] \odot d \log p\right)^{\prime}
$$

\section{Term 2:}

$$
\left(\frac{\xi_{j}-\varepsilon}{\Omega s_{j}^{k c}}\right) \sum_{v \in \mathcal{C}} \Omega_{j v}^{k c} d \log p_{j v}
$$


In Matrix notation:

$$
O T_{(j)}^{2}=1_{C N}^{\prime} \otimes\left[\left(\xi_{j}-\sigma\right)\left(\Omega_{(j)}^{\mathcal{N}} \oslash \Omega s\right) d \log p_{(j)}\right]
$$

Term 3:

$$
\left(\frac{\varepsilon-\phi}{1-\alpha_{k c}}\right) \sum_{i v \in \mathcal{C N}} \Omega_{i v}^{k c} d \log p_{i v}
$$

In Matrix notation:

$$
O T^{3}=(\varepsilon-\phi) 1_{C N}^{\prime} \otimes\left(\left[\Omega^{\mathcal{N}} \oslash(1-\alpha)\right] d \log p\right)
$$

Term 4:

$$
(\phi-1) d \log p_{k c}
$$

In Matrix notation:

$$
O T^{4}=(\phi-1)\left(d \log p \otimes 1_{C N}^{\prime}\right)
$$

Changes in value-added share:

$$
d \Omega_{f_{k c}}^{k c}=d \alpha_{k c}=\alpha_{k c}(1-\phi) d \log w_{f_{k c}}+\alpha_{k c}(\phi-1) d \log p_{k c} .
$$

In Matrix notation:

$$
d \alpha=(\phi-1)(\alpha \odot[d \log p-d \log w]) \otimes 1_{C N}^{\prime}
$$

Leontief Inverse: After combining $d b$ and $d \Omega$ matrices, we can write $(C+C N+C F) \times$ $(C+C N+C F)$ dimensional $d \Psi$ as:

$$
d \Psi=\Psi d \Omega \Psi
$$

Good Domar Weights: Recall that:

$$
d \lambda^{\prime}=\left(d \chi^{\prime} b+\chi^{\prime} d b+\lambda^{\prime} d \Omega\right) \Psi .
$$

We know that $d \chi^{\prime}, d b$ and $d \Omega$ terms from above. Hence, we can calculate the changes in Good Domar weights from this expression. 